\title{
FINAL TECHNICAL REPORT \\ for \\ 1993
}

Work performed under Subcontract Number 9-XG2-W3173-1

for

Los Alamos National Laboratory

by

A.K. Kapila

Rensselaer Polytechnic Institute

Troy, New York 12180-3590

\section{DISCLAIMER}

This report was prepared as an account of work sponsored by an agency of the United States Government. Neither the United States Government nor any agency thereof, nor any of their employees, makes any warranty, express or implied, or assumes any legal liability or responsibility for the accuracy, completeness, or usefulness of any information, apparatus, product, or process disclosed, or represents that its use would not infringe privately owned rights. Reference herein to any specific commercial product, process, or service by trade name, trademark, manufacturer, or otherwise does not necessarily constitute or imply its endorsement, recommendation, or favoring by the United States Government or any agency thereof. The views and opinions of authors expressed herein do not necessarily state or reflect those of the United States Government or any agency thereof.

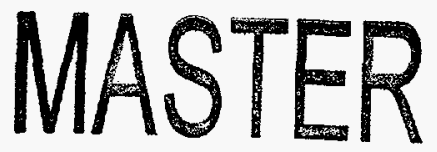




\section{CONTENTS}

1. The Multi-phase Equations in One Dimension

Nondimensionalization and characteristic analysis.

2. On the Solid Equation of State

An inconsistency in the BN model.

3. Asymptotic Reduction of Two-Phase DDT Models

Reducing the 7-equation formulation via velocity equilibrium and temperature equilibrium, to assess relationship to the SPA model.

4. Characteristic Analyses of the Reduced Models

Includes the velocity-equilibrium model (BKS), temperature-equilibrium model (SPAlike) and the pressure-equilibrium model.

5. Compaction-wave computations with Prasad's Code

Includes velocity-equilibrium and temperature-equilibrium cases. This computation was followed by Prasad's departure from the group. The remainder of the year was spent mostly in the development of the AUGUST and SEPTEMBER codes in collaboration with D.S. Stewart.

6. On the six-equation Equivelocity Model

A clarification of the way in which an equivelocity model may be constructed: setting $v_{s}=v_{g}$ a priori, versus deriving the model as a consequence of the large-drag assumption.

7. The Six-equation, Equivelocity Model: Scaling Argues for a scaled version of SEPTEMBER, and suggests appropriate scales. 


\section{ITEM 1}




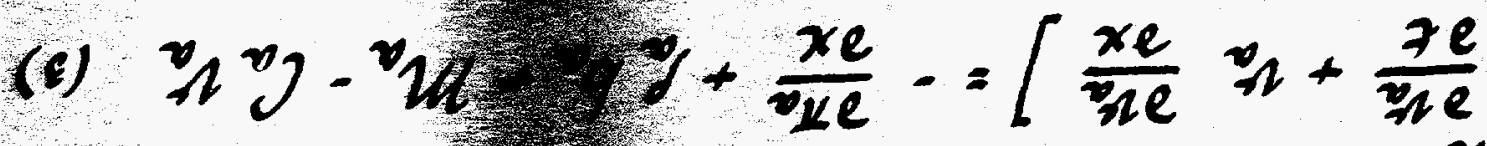

unzunies

(z)

$$
=\frac{x_{e}}{x_{l}} y+\frac{x_{e}}{y_{e}} w_{n}+
$$

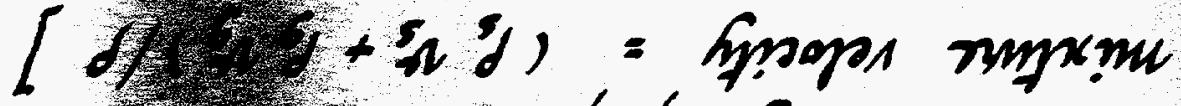
t.

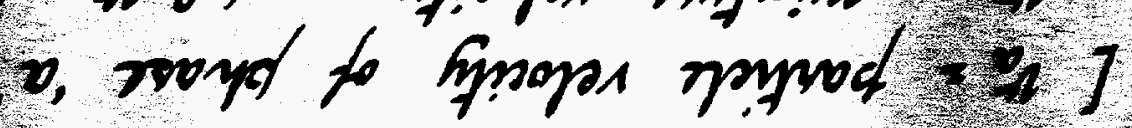

(I)

$$
1=6 \phi+\phi)
$$

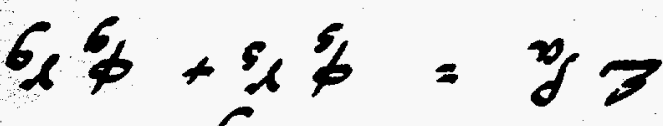
hpromo numprme aroyd to rusond anyol

$$
\text { if } \%
$$

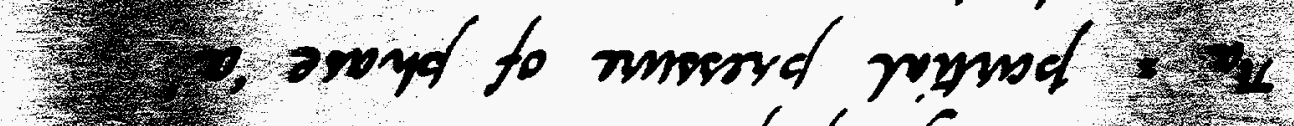

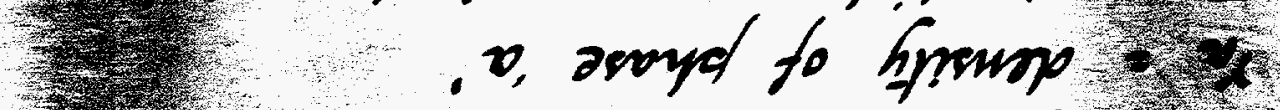
63
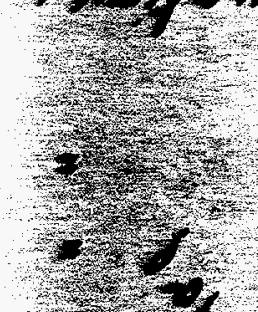

중 $=$

$$
\text { 15. } \quad \boldsymbol{y}_{\mathbf{\gamma}} \mathbf{\phi}=
$$

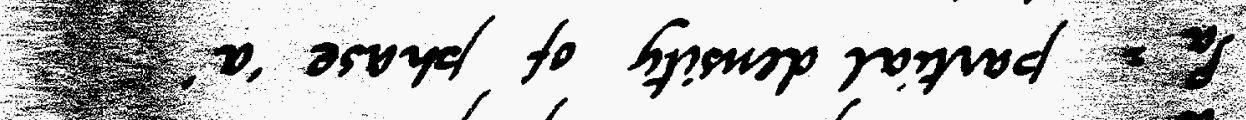

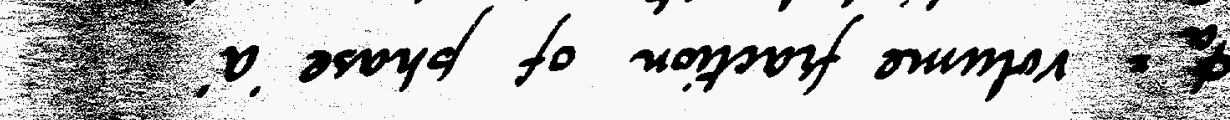
$=199700 \mathrm{~N}$ 
Additional notation:

$b_{a}=$ body free / mass

$\mathcal{C}_{a}=$ mass source for phase ' $a$ ', mass/ral. - time

$m_{a}=$ momentum sores for phase ' $a$;' mom./mt. - t

$e_{a}=$ specific internal energy

$q_{a}=$ heat flux

$r_{a}=$ external energy seance, energy/mas-time

$\varepsilon_{a}=$ internal energy sones, encysy/val. - time

Notes: 1. The balance equations follow those for a single fluid.

2. The summing rules $C_{g}+C_{b}=0, M_{g}+M_{b}=0$, $\varepsilon_{g}+\varepsilon_{b}=0$, hold.

The above eons, are supplemented by the compaction en.

$$
\frac{\partial \phi_{s}}{\partial t}+v_{s} \frac{\partial \phi_{s}}{\partial x}=F+\frac{\mathcal{C}_{s}}{\gamma_{s}}
$$

Constitutive information:

$$
\begin{aligned}
F= & \frac{1}{\mu_{c}} \phi_{s} \phi_{g}\left(p_{s}-p_{s}-\beta\right) \\
q_{s}= & -k_{a} \frac{\partial T_{a}}{\partial x} \\
m_{p}= & p_{g} \frac{\partial \phi_{s}}{\partial x}-D+\mathcal{C}_{s} v_{s} \\
D= & \left(\delta+\frac{1}{2} \mathcal{C}_{s}\right)\left(v_{s}-v_{g}\right) \\
\varepsilon_{s}= & {\left[\phi_{g} \frac{\partial \phi_{s}}{\partial x}-D\right] v_{s}-\left(p_{s}-\beta\right) F+H } \\
& +\mathcal{C}_{s}\left(e_{s}+\frac{1}{2} v_{s}^{2}\right) \\
H= & h\left(T_{g}-T_{s}\right)
\end{aligned}
$$




\section{DISCLAMMER}

Portions of this document may be illegible in electronic image products. Images are produced from the best available original document. 
3.

Additional notation still:

$\delta=$ dry coefficient

$h=$ heat transfer coefficient

$k_{a}=$ thermal conductivity

$\mu_{c}=$ compaction viscosity

$\beta_{s}=$ solid configuration pressure

Notes: 1. The choices made above for the constitutive equs. are sufficient (but not neecsany) to ensure the satisfaction of the entropy inequality. This is where the model is at its weakest.

2. The configuration pressure $\beta$ is a measure of the contact forces between the solid greene:

3. When $\mu_{c}$ vanishes, the compaction equation reduces to $p_{s}=p_{g}+\beta_{6}$.

Let us now suppose that

$$
b_{a}=N_{a}=0 \text {. }
$$

(I)

Then, by using the constitutive information, the balance laws can be rewritten.

Solid mass balance:

$$
\begin{aligned}
& \frac{\partial}{\partial t}\left(\phi_{p} r_{p}\right)+v_{p} \frac{\partial}{\partial x}\left(\phi_{p} r_{p}\right)=c_{s}-\phi_{p} r_{p} \frac{\partial v_{p}}{\partial x} \\
& \Rightarrow \phi_{s}\left\{\frac{\partial Y_{s}}{\partial t}+v_{p} \frac{\partial Y_{s}}{\partial x}\right\}+Y_{p}\left\{\frac{\partial \phi_{s}}{\partial t}+v_{p} \frac{\partial \phi_{s}}{\partial x}\right\}=-\phi_{s} V_{s} \frac{\partial V_{s}}{\partial x}
\end{aligned}
$$

use of $(2.1) \Rightarrow$

$$
\frac{\partial r_{s}}{\partial t}+v_{s} \frac{\partial r_{s}}{\partial x}=-\frac{r_{s}}{\phi_{s}} F-r_{s} \frac{\partial v_{s}}{\partial x} .
$$


Gas mass balance:

$$
\begin{aligned}
\frac{\partial}{\partial t}\left(\phi_{g} r_{g}\right)+v_{g} \frac{\partial}{\partial x}\left(\phi_{g} r_{g}\right)+\phi_{g} r_{g} \frac{\partial v_{g}}{\partial x}=-\mathcal{C}_{p} \\
\Rightarrow \quad \phi_{g}\left\{\frac{\partial r_{g}}{\partial t}+v_{g} \frac{\partial r_{g}}{\partial x}\right\}+r_{g}\left\{\frac{\partial \phi_{g}}{\partial t}+v_{g} \frac{\partial \phi_{g}}{\partial x}\right\} \\
=-c_{p}-\phi_{g} r_{g} \frac{\partial v_{g}}{\partial x}
\end{aligned}
$$

On using $\dot{\phi}_{g}=-\dot{\phi}_{s}, \Rightarrow$

$$
\begin{aligned}
\frac{\partial r_{g}}{\partial t}+v_{g} \frac{\partial r_{g}}{\partial x}-\frac{r_{g}}{\phi_{g}} & \left\{\frac{\partial \phi_{s}}{\partial t}+v_{p} \frac{\partial \phi_{g}}{\partial x}+\left(v_{g}-v_{p}\right) \frac{\partial \phi_{g}}{\partial x}\right. \\
& =-\frac{c_{p}}{\phi_{g}}-r_{g} \frac{\partial v_{g}}{\partial x}
\end{aligned}
$$

On using (2.1), $\Rightarrow$

$$
\begin{aligned}
\frac{\partial r_{g}}{\partial t}+v_{g} \frac{\partial r_{g}}{\partial x}= & -r_{g} \frac{\partial v_{g}}{\partial x}-\frac{r_{g}}{\phi_{g}}\left(v_{p}-v_{g}\right) \frac{\partial \phi_{g}}{\partial x}+\frac{r_{g}}{\phi_{g}}= \\
& -\left(1-\frac{r_{g}}{r_{p}}\right) \frac{c_{f}}{\phi_{g}} .
\end{aligned}
$$

Solid momentum e balance:

$\phi_{p} \gamma_{s}\left(\frac{\partial v_{s}}{\partial t}+v_{s} \frac{\partial v_{s}}{\partial x}\right)=-\phi \frac{\partial p_{s}}{\partial x}-p_{p} \frac{\partial \phi_{s}}{\partial x}+p_{p} \frac{\partial \phi_{s}}{\partial x}-D$

(on using $D) \Rightarrow$

$\phi_{s} \gamma_{s}\left(\frac{\partial v_{s}}{\partial t}+v_{s} \frac{\partial v_{s}}{\partial x}\right)=-\phi_{p} \frac{\partial p_{s}}{\partial x}+\left(p_{p}-p_{p}\right) \frac{\partial \phi_{s}}{\partial x}-\left(\delta+\frac{\partial}{2} C_{p}\right) d$

Gas momentum balance:

$$
\begin{gathered}
\phi_{g} r_{g}\left(\frac{\partial v_{g}}{\partial t}+v_{g} \frac{\partial v_{g}}{\partial x}\right)=-\phi_{g} \frac{\partial p_{g}}{\partial x}-p_{g} \frac{\partial \phi_{g}}{\partial x}-m_{p}+\mathcal{C}_{p} \\
=-\phi_{g} \frac{\partial p_{g}}{\partial x}+\Delta-\mathcal{C}_{s} v_{p}+\mathcal{C}_{s} v_{g}
\end{gathered}
$$




$$
\phi_{g} \gamma_{g}\left(\frac{\partial v_{g}}{\partial t}+v_{g} \frac{\partial v_{g}}{\partial x}\right)=-\phi_{g} \frac{\partial p_{g}}{\partial x}+\left(\mathcal{\delta}-\frac{1}{2} \mathcal{C}_{\mathcal{S}}\right)\left(v_{p}-v_{g}\right) .
$$

Solid energy balance:

$$
\begin{gathered}
\phi_{p} y_{p}\left(\frac{\partial e_{s}}{\partial t}+v_{p} \frac{\partial e_{p}}{\partial x}\right)=-\phi_{p} \phi_{p} \frac{\partial v_{s}}{\partial x}+\frac{\partial}{\partial x}\left(k_{p} \frac{\partial T_{p}}{\partial x}\right) \\
+\varepsilon_{p}-m_{\rho} v_{p}-\left(e_{p}-\frac{1}{2} v_{p}^{2}\right) c_{p} .
\end{gathered}
$$

Now,

$$
\begin{aligned}
& \varepsilon_{p}-m_{p} v_{p}-\left(e_{p}-\frac{1}{2} v_{p}^{2}\right) c_{p} \\
& =\left[p_{g} \frac{\partial \phi_{p}}{\partial x}-\left(\delta+\frac{1}{2} c_{p}\right)\left(v_{p}-v_{g}\right)\right] v_{s}-\left(\phi_{p}-\beta_{p}\right) F \\
& +H+c_{p}\left(e_{p}+\frac{1}{2} v_{p}^{2}\right)-\left[p_{p} \frac{\partial \phi_{p}}{\partial x}-0\right] v_{\beta}-c_{p} v_{p}^{2} \\
& -\left(e_{p}-\frac{1}{2} v_{\rho}^{2}\right) c_{\rho} \\
& =h\left(T_{g}-T_{p}\right)-\left(p_{p}-\beta_{p}\right) F
\end{aligned}
$$

$\boldsymbol{s}$

solid enagy balauce $\rightarrow$

$$
\begin{aligned}
& \phi_{p} \eta_{p}\left(\frac{\partial e_{p}}{\partial t}+v_{p} \frac{\partial e_{s}}{\partial x}\right)=-\phi_{p} \phi_{p} \frac{\partial v_{p}}{\partial x}+\frac{\partial}{\partial x}\left(k_{p} \frac{\partial T_{s}}{\partial x}\right) \\
& -h\left(T_{p}-T_{g}\right)-\left(p_{\beta}-\beta_{s}\right) F \text {. }
\end{aligned}
$$

Gas enengy balance:

$$
\begin{aligned}
& \phi_{g} V_{g}\left(\frac{\partial e_{g}}{\partial t}+v_{g} \frac{\partial e_{g}}{\partial x}\right)=-\phi_{g} p_{g} \frac{\partial v_{g}}{\partial x}+\frac{\partial}{\partial x}\left(k_{g} \frac{\partial T_{g}}{\partial x}\right) \\
&-E_{g}+m_{p} v_{g}+\left(e_{g}-\frac{1}{\partial} v_{g}^{2}\right) C_{s} \\
&=-\phi_{g} p_{g} \frac{\partial v_{g}}{\partial x}+\frac{\partial}{\partial x}\left(k_{g} \frac{\partial T_{g}}{\partial x}\right)+k\left(T_{p}-T_{g}\right)+\left(p_{p}-p_{p}\right) F \\
&-\left(v_{p}-v_{g}\right) p_{g} \frac{\partial q_{p}}{\partial x}+\delta\left(v_{p}-v_{g}\right)^{2}-\left(e_{p}-e_{g}\right) C_{p} .
\end{aligned}
$$


Equations of state:

So fac we have 12 unknowns $\left(e_{a}, T_{a}, v_{a}, Y_{a}, t_{a}\right.$, and s equations. The deficit is made up by the equation of state. If one uses the canonical aqua. of state, the

$$
e_{a}=e_{a}\left(\eta_{a}, r_{a}\right)
$$

where $\eta_{a}$ is the entropy, whence

$$
T_{a}=\frac{\partial e_{a}}{\partial \eta_{a}}, p_{a}=\gamma_{a}^{2} \frac{\partial e_{a}}{\partial r_{a}} \text {. }
$$

Alternatively one could use a thermal equation of et.

$$
p_{a}=p_{a}\left(r_{a}, T_{a}\right)
$$

and a caloric equation of state,

$$
e_{a}=e_{a}\left(r_{a}, T_{a}\right) \text { ? }
$$

let us choose the latter path and pet

$$
e_{a}=c_{v a} T_{a}, \quad p_{a}=\Gamma_{a} c_{v a} r_{a} T_{a}
$$

Here $\Gamma_{a}$ is the Greisen coefficient. (Essentially, each phase is treated like an ideal gas.)

We ale treat the contact stress $\beta$ to be a prescribed function of $\phi$ :

$$
\beta_{\beta}=\beta\left(\phi_{\beta}\right) \text {. }
$$


7.

Summary of equations at this stage:

Mass

$$
\begin{aligned}
& \frac{\partial r_{s}}{\partial t}+v_{s} \frac{\partial r_{s}}{\partial x}=-\frac{r_{s}}{\phi_{s}}=-r_{s} \frac{\partial v_{s}}{\partial x} \quad \frac{\partial \phi_{s}}{\partial x} \\
& \frac{\partial r_{g}}{\partial t}+v_{g} \frac{\partial r_{g}}{\partial x}=+\frac{r_{g}}{\phi_{g}} F-r_{g} \frac{\partial v_{g}}{\partial x}-\frac{r_{g}}{\phi_{g}}\left(v_{s}-v_{g}\right)-\left(1-\frac{r_{g}}{r_{s}}\right) \frac{C_{s}}{\phi_{g}} .
\end{aligned}
$$

Momentum

$$
\begin{aligned}
& \phi_{s} \psi_{s}\left(\frac{\partial v_{s}}{\partial t}+v_{s} \frac{\partial v_{s}}{\partial x}\right)=-\phi_{s} \frac{\partial \phi_{s}}{\partial x}+\left(\phi_{g}-p_{p}\right) \frac{\partial \phi_{s}}{\partial x}-\left(\delta+\frac{\partial}{2} \mathcal{C}_{s}\right) x \\
& x\left(v_{p}-v_{y}\right) \\
& \phi_{g} Y_{g}\left(\frac{\partial v_{g}}{\partial t}+v_{g} \frac{\partial v_{g}}{\partial x}\right)=-\phi_{g} \frac{\partial p_{g}}{\partial x}+\left(\delta-\frac{1}{2} C_{s}\right)\left(v_{s}-v_{g}\right)
\end{aligned}
$$

Energy

$$
\begin{aligned}
& \phi_{s} y_{p}\left(\frac{\partial e_{s}}{\partial t}+v_{s} \frac{\partial e_{s}}{\partial x}\right)=-\phi_{s} p_{p} \frac{\partial v_{s}}{\partial x}+\frac{\partial}{\partial x}\left(k_{s} \frac{\partial T_{s}}{\partial x}\right) \\
& -h\left(T_{p}-T_{g}\right)-\left(p_{\beta}-\beta\right) F . \\
& \phi_{g} r_{g}\left(\frac{\partial e_{g}}{\partial t}+v_{g} \frac{\partial e_{g}}{\partial x}\right)=-\phi_{g} g_{g} \frac{\partial v_{g}}{\partial x}+\frac{\partial}{\partial x}\left(k_{g} \frac{\partial T_{g}}{\partial x}\right) \\
& +h\left(T_{p}-T_{g}\right)+\left(\phi_{p}-\beta_{p}\right) F \\
& -\left(v_{p}-v_{g}\right) \psi_{g} \frac{\partial \phi_{s}}{\partial x}+\delta\left(v_{s}-v_{g}\right)^{2} \\
& -\left(e_{\beta}-e_{g}\right) \mathcal{C}_{\beta} \text {. }
\end{aligned}
$$

Compaction

$$
\frac{\partial \phi_{\beta}}{\partial t}+V_{\beta} \frac{\partial \phi_{s}}{\partial x}=F+\frac{\mathcal{C}_{\beta}}{Y_{s}} .
$$




$$
\begin{aligned}
& F=\frac{\phi_{p} \phi_{g}}{\mu_{c}}\left[p_{b}-\beta_{b}-p_{g}\right] \\
& \phi_{g}=1-\phi_{b} \\
& e_{a}=c_{r a} T_{a}, p_{a}=\Gamma_{a} C_{v a} r_{a} T_{a}
\end{aligned}
$$

It remains to specify $C_{\beta}$. The units of $C_{s}$ are mass/vele-tume. Let us assume that

$$
\mathcal{C}_{\beta}=\kappa r_{\beta} \Phi
$$

where $\Phi$ is a yet unspecified, dimensionless fence and $K$ has units of if time.

Sonic speed $a_{p}^{2}=(p+1) \frac{p_{s}}{r_{p}}$

(5)

$$
a_{g}^{y}=\left(r_{g}+1\right) \frac{p_{g}}{r_{g}}
$$


9

Nondimensionalization

Reference quantities:

$$
\bar{p}, \bar{r}, \quad \bar{v}=\sqrt{\bar{p} / \bar{r}}, \bar{t}, \bar{x}=\bar{v} \bar{k}, \bar{T}
$$

Dimensionless parameters:

$$
\begin{aligned}
& \tilde{\mu}_{c}=\frac{\mu_{c}}{\bar{F} \bar{F}}, \\
& \tilde{h}=\bar{t} k \\
& \tilde{\delta}=\frac{\bar{t}}{\bar{r} \delta} \\
& \tilde{c}_{v a}=\frac{\bar{r} \bar{F}}{\bar{p}} c_{v a} \\
& \tilde{k}=\frac{\bar{F}}{\bar{p} \bar{v} \bar{x}} k_{a} \\
& \tilde{h}=\frac{\bar{F} \bar{x}}{\bar{p} \bar{v}}
\end{aligned}
$$


Dimensionless Equations

$$
\begin{aligned}
& F=\frac{\phi_{0} \phi_{g}}{\tilde{\mu}_{c}}\left(p_{p}-\beta_{p}-p_{g}\right) \\
& \phi=1-\phi_{b} \\
& \hat{p}_{a}=r_{a} \tilde{c}_{v a} r_{a} T_{a}
\end{aligned}
$$

mass

$$
\begin{aligned}
& \frac{\partial x_{0}}{\partial t}+v_{s} \frac{\partial r_{s}}{\partial x}=-\frac{r_{s}}{\phi_{s}} F-r_{p} \frac{\partial}{\partial z} \\
& \frac{\partial r_{g}}{\partial t}+t_{g} \frac{\partial r_{g}}{\partial x}=\frac{r_{g}}{g} F-r_{g} \frac{\partial u_{g}}{\partial x} \\
& -\frac{1}{\phi_{g}}\left(1-\frac{r_{g}}{r_{0}}\right) \tilde{r} r_{0}
\end{aligned}
$$

va) $\frac{\partial y}{\partial x}$

momentum

$$
\begin{aligned}
& \phi_{p} \gamma_{p}\left(\frac{\partial v_{p}}{\partial t}+v_{p} \frac{\partial v_{s}}{\partial x}\right)=-\phi_{s} \frac{\partial p_{p}}{\partial x}+(f)
\end{aligned}
$$

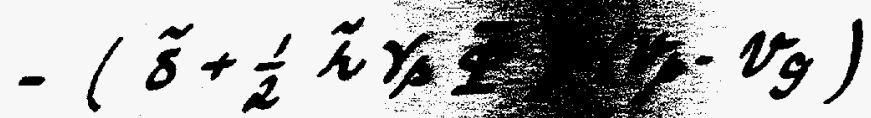

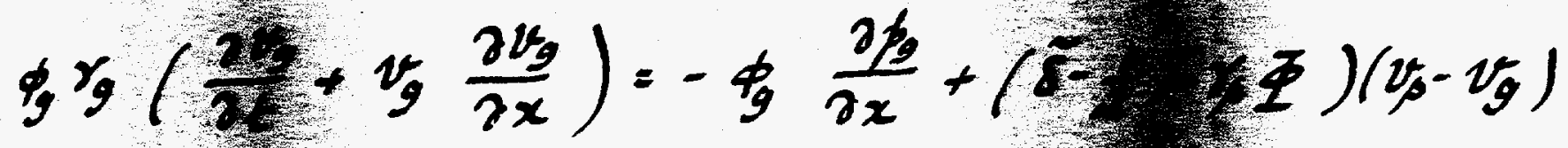

energy

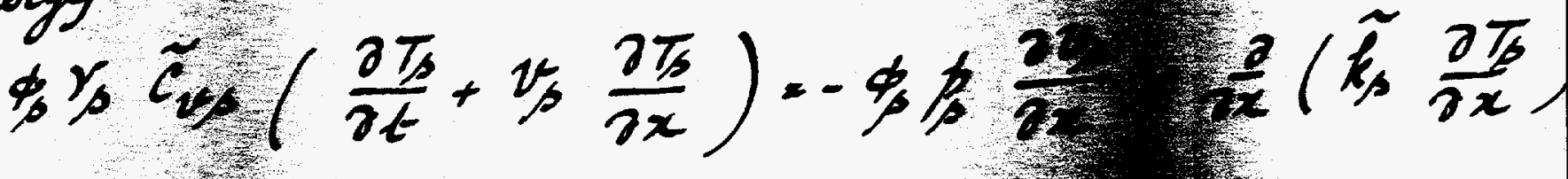

$$
\begin{aligned}
& \left.-\tilde{h}\left(T_{p}-T_{y}\right)+1\right) F
\end{aligned}
$$

SHS can also be wrilture as

$$
\phi f t\left(\frac{\partial e_{s}}{\partial t}+v_{s} \frac{\partial e_{s}}{\partial x}\right)
$$


$1 \%$

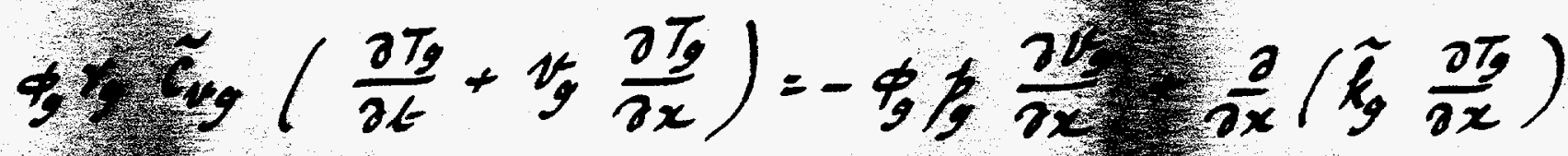

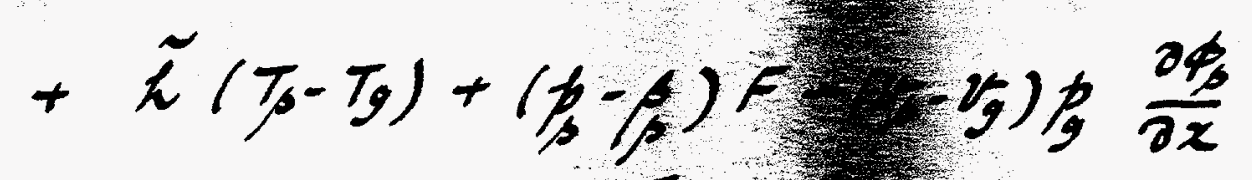

$+\tilde{\delta}\left(v_{p}-v_{g}\right)^{2}-\left(\tilde{c}_{v p h}^{T_{p}}-\tilde{c}_{v g}\right) \tilde{r} r_{p} \Phi$

(I)

comptip in

$$
\frac{\partial \phi_{p}}{\partial t}+v_{s} \frac{\partial \phi_{s}}{\partial x}=F+\tilde{i} \Phi
$$

we 4 choose the basic variables.

$\left(r_{p}, v_{s}, p_{p}, \phi_{p}, r_{g}, v_{p}, p_{0}\right.$

(3)

and ftempt to wite the abore $F$ as

$$
\left.\frac{\partial f}{\partial}+\underline{A}(\underline{u}) \frac{\partial \underline{u}}{\partial x}=\underline{R}(\underline{u})+\frac{\hat{L}}{\partial t}(\underline{y}) \frac{\partial \underline{u}}{\partial x}\right) \text {. }
$$

Finst rote that

$$
\begin{aligned}
& =r_{a} \tilde{c}_{v a} r_{a} T_{a} \Rightarrow \\
& =r_{a} \tilde{c}_{v a}\left(r_{a} d T_{a}+T_{a} d ?\right.
\end{aligned}
$$

Shere

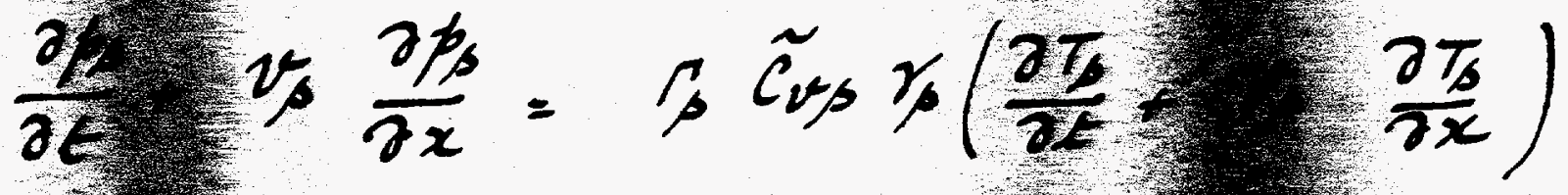

$$
\begin{aligned}
& +T_{\beta} \tilde{c}_{\nu p} T_{p}\left(\frac{\partial x_{s}}{\partial t}\right)\left(\frac{\partial r_{s}}{\partial x}\right)
\end{aligned}
$$


On using (10.4) and (10.8),

$$
\begin{aligned}
& \frac{\partial p_{s}}{\partial t}+\frac{\partial p_{s}}{\partial x}=\frac{F_{s}}{\phi_{s}}\left[-\phi_{p} p_{s} \frac{\partial v_{s}}{\partial x}+\frac{\partial}{\partial x}\left(\tilde{k}_{p} \frac{\partial T_{s}}{\partial x}\right)\right. \\
& \left.-\tilde{z}\left(T_{p}-T_{g}\right)-\left(p_{p}-p_{p}\right) F\right] \\
& +\Gamma_{\beta} \tilde{c_{v p}} T_{p}\left[-\frac{r_{s}}{\phi_{s}} F-r_{p} \frac{\partial v_{s}}{\partial x}\right] \\
& =-\left(1+\Gamma_{p}\right) \frac{\partial v_{p}}{\partial x}+\frac{\Gamma_{p}}{\phi_{p}}\left[-\tilde{h}\left(T_{p}-T_{g}\right)-\left(\phi_{p}-\beta_{p}\right) F\right. \\
& -\frac{\partial}{f} F+\frac{\rho_{\beta}}{\partial x} \frac{\partial}{\partial x}\left(\tilde{k}_{p} \frac{\partial T_{j}}{\partial x}\right)
\end{aligned}
$$

$\infty$,

$$
\begin{aligned}
& \frac{\partial p_{s}}{\partial t}+v_{p} \frac{\partial p_{s}}{\partial z}+\left(1+\Gamma_{p}\right) p_{p} \frac{\partial v_{s}}{\partial x}=-\frac{F_{p}}{\phi_{p}} \tilde{L}\left(T_{p}-T_{g}\right)
\end{aligned}
$$

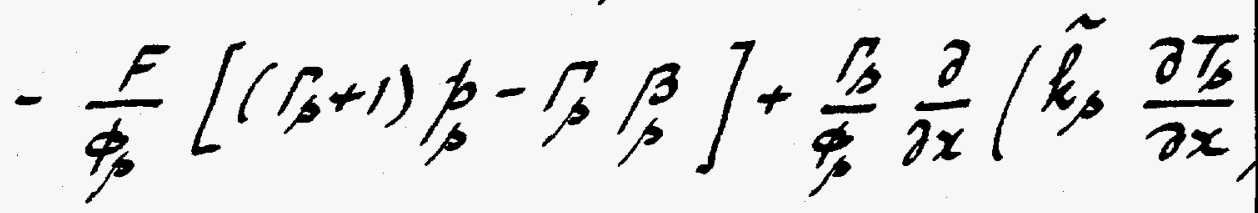

similarly,

$$
\begin{aligned}
& \frac{\partial p_{g}}{\partial t}+v_{g} \frac{\partial p_{g}}{\partial x}=\frac{\rho_{g}}{\phi_{g}} \int-\phi_{g} p_{g} \frac{\partial v_{g}}{\partial x}+\frac{\partial}{\partial x}\left(\tilde{k} \frac{\partial T_{g}}{\partial x}\right) \\
& +\tilde{z}\left(T_{p}-T_{g}\right)+\left(p_{p}-\beta_{\beta}\right) F-\left(v_{p}-v_{g}\right) p_{g} \frac{\partial \phi_{p}}{\partial x} \\
& \left.+\tilde{\varepsilon}\left(v_{p}-v_{g}\right)^{2}-\left(\tilde{c}_{v p s} T_{p}-\tilde{c_{v g}} T_{g}\right) \tilde{r} r_{s} \Phi\right]
\end{aligned}
$$

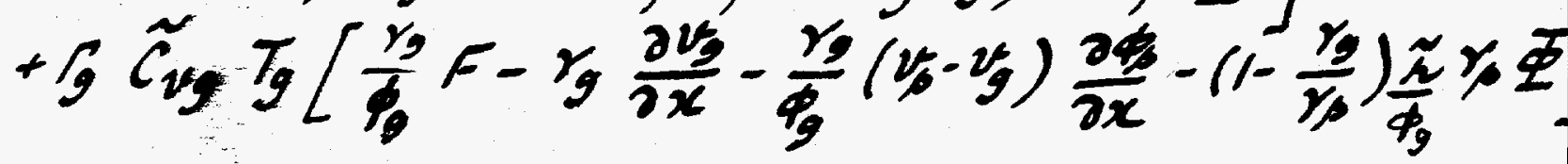


13

$$
\begin{aligned}
& \frac{\partial p_{g}}{\partial t}+v_{g} \frac{\partial p_{g}}{\partial x}+\left(1+r_{g}\right) p_{g} \frac{\partial v_{g}}{\partial x}+\left(1+r_{g}\right) \frac{p_{g}}{p_{g}}\left(v_{p}-v_{g}\right) \frac{\partial \phi_{g}}{\partial x} \\
& =\frac{F_{g}}{\phi_{g}}\left[\tilde{h}\left(T_{p}-T_{g}\right)+\tilde{\delta}\left(v_{p}-v_{g}\right)^{2}+\left(p_{p}-p_{p}\right) F\right. \\
& \left.-\left(\tilde{c}_{v p} T_{p}-\tilde{C}_{v g} T_{g}\right) \tilde{r} r_{p} \Phi\right] \\
& +\frac{p_{g}}{\phi_{g}}=-\frac{1}{g_{g}} \tilde{g} \tilde{c}_{n g} T_{g}\left(1-\frac{r_{g}}{r_{p}}\right) \tilde{r} r_{p} \Phi . \\
& +\frac{\sqrt{g}}{\phi g} \frac{\partial}{\partial x}\left(\tilde{k} \frac{\partial T g}{\partial x}\right) \text {. }
\end{aligned}
$$

we can now wite down the matrix $\stackrel{A}{=}$ introduced in (11.4):

first, rewrite the governing quo. the order as

$$
\begin{aligned}
& \frac{\partial r_{s}}{\partial t}+v_{s} \frac{\partial r_{s}}{\partial x}+r_{p} \frac{\partial v_{s}}{\partial x}=R_{1} \\
& \frac{\partial v_{p}}{\partial t}+v_{s} \frac{\partial v_{s}}{\partial x}+\frac{1}{y_{p}} \frac{\partial p_{s}}{\partial x}+\frac{p_{p}-p_{g}}{\partial y_{p}} \frac{\partial \phi_{p}}{\partial x}=R_{2} \\
& \frac{\partial p_{p}}{\partial t}+v_{p} \frac{\partial p_{s}}{\partial x}+\left(1+F_{p}\right) p_{p} \frac{\partial v_{p}}{\partial x}=R_{\beta}+\frac{F_{p}}{\partial x} \frac{\partial}{\partial x}\left(\tilde{k}_{p} \frac{\partial T_{p}}{\partial x}\right) \\
& \frac{\partial \phi_{p}}{\partial t}+v_{p} \frac{\partial \phi_{p}}{\partial x}=R_{4} \\
& \frac{\partial r_{g}}{\partial t}+v_{g} \frac{\partial r_{g}}{\partial x}+r_{g} \frac{\partial v_{g}}{\partial x}+\frac{r_{g}}{g_{g}}\left(v_{p}-v_{g}\right) \frac{\partial \Phi_{g}}{\partial x}=R_{g} \\
& \frac{\partial v_{g}}{\partial t}+v_{g} \frac{\partial v_{g}}{\partial x}+\frac{1}{\gamma_{g}} \frac{\partial p_{g}}{\partial x}=R_{6} \quad \frac{\partial}{\partial x}\left(\tilde{k_{g}} \frac{\partial T_{g}}{\partial x}\right) \\
& \frac{\partial p_{g}}{\partial t}+v_{g} \frac{\partial p_{g}}{\partial x}+\left(1+f_{g}\right) p_{g} \frac{\partial v_{g}}{\partial x}+\left(1+t_{g}\right) \frac{p_{g}}{g}\left(v_{p}-v_{g}\right) \frac{\partial \phi_{g}}{\partial x}=R_{7}+\frac{F_{g}}{g_{g}}(
\end{aligned}
$$




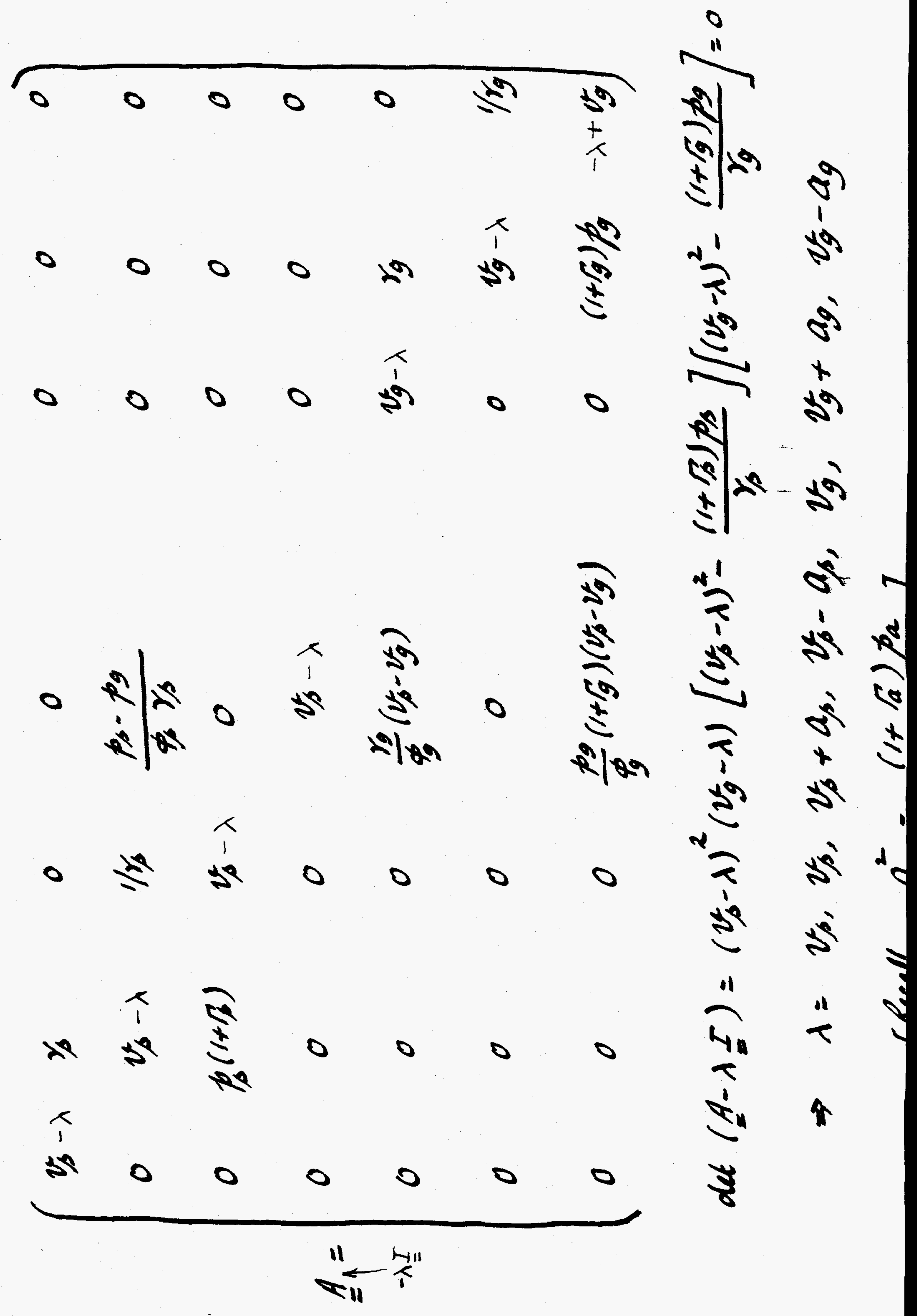


15.

With the eigenvalues known, let us compute the left eigenvectors, which satisfy

$$
\left(\stackrel{A}{ }^{\top}-\lambda \underline{I}\right) \underline{l}=\underline{0} \text {. }
$$

(i) $\lambda=\lambda_{1}=v_{\beta}$

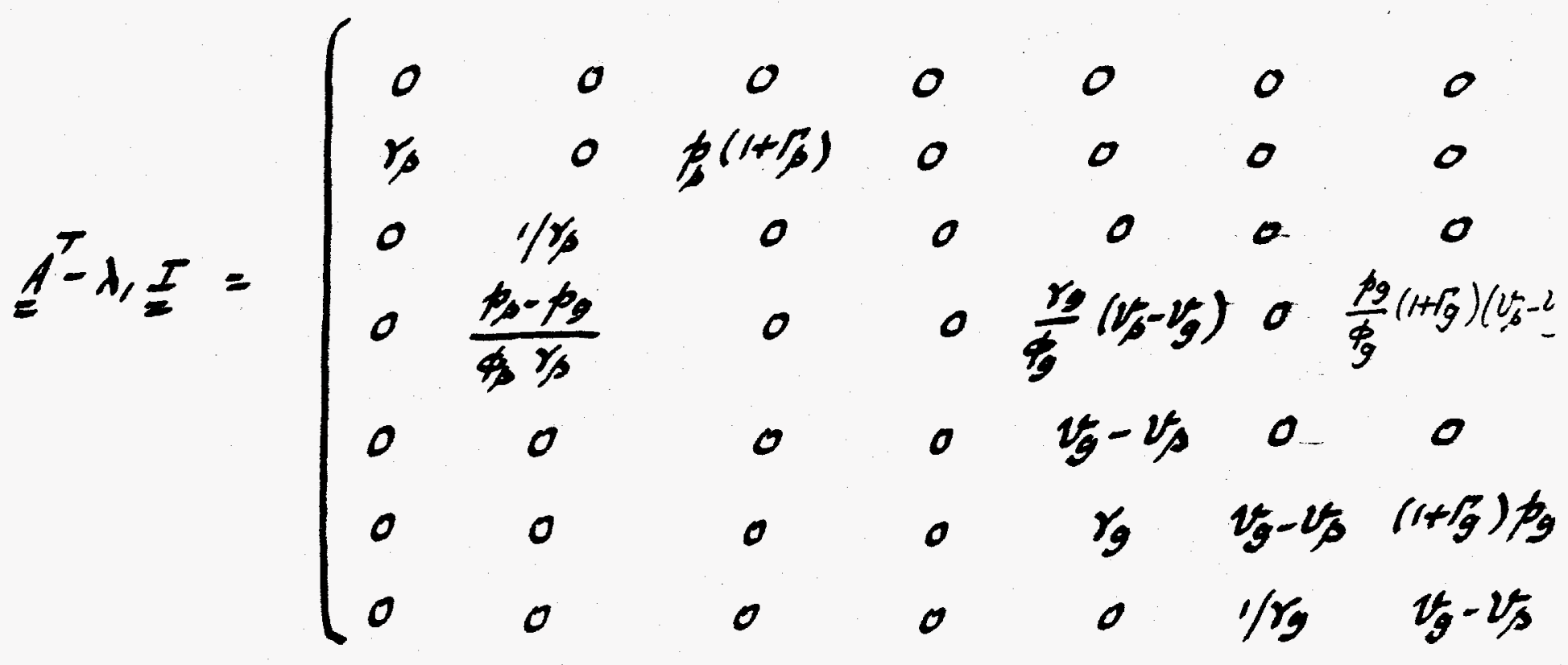

$\left(A^{\top}-\lambda, I\right) \underline{l}=0$ reduces to

$$
\begin{gathered}
r_{p} l_{1}+p_{s}\left(1+r_{p}\right) l_{3}=0 \\
l_{2}=0 \\
\frac{1}{\phi_{g}}\left(v_{b}-v_{g}\right)\left[r_{g} l_{g}+p_{g}\left(1+l_{g}\right) l_{7}\right]=0 \\
\left(v_{g}-v_{s}\right) l_{s}=0 \\
r_{g} l_{g}+\left(v_{g}-v_{p}\right) l_{b}+\left(1+l_{g}\right) p_{g} l_{7}=0 \\
\frac{1}{r_{g}} l_{b}+\left(v_{g}-v_{s}\right) l_{7}=0
\end{gathered}
$$

There are two perceibilitis

(a) $\quad v-v_{p} \neq 0$. Now, $\lambda=v_{\%}$ has multiplicity 2 , 
$\therefore$ e, $\quad \lambda_{1}=\lambda_{2}=\nu_{\%}$.

We thur get $l_{2}=l_{5}=l_{6}=l_{7}=0, l_{1}=-a_{3}^{2} l_{3}$

$$
\begin{aligned}
\because \underline{l}^{\top}= & \left(-a_{3}^{2} l_{3}, 0, l_{3}, l_{4}, 0,0,0\right) \\
= & l_{3}\left(-a_{3}^{2}, 0,1,0,0,0,0\right) \\
\text { and } & l_{4}(0,0,0,1,0,0,0)
\end{aligned}
$$

(b) $v_{g}-v_{p}=0 . \quad$ Now, $A=v_{p}$ has multiplicity 3. J

$$
\underline{l}^{\top}=\left(-a_{3}^{2} l_{3}, 0, l_{3}, l_{4},-a_{9}^{2} l_{7}, 0, l_{7}\right) \text {, }
$$

yielding the additional eigenvector $\left(0,0,0,0,-a_{g}^{2}, 0,1\right)$.

(ii) $\lambda=v_{p} \mp a_{s}$

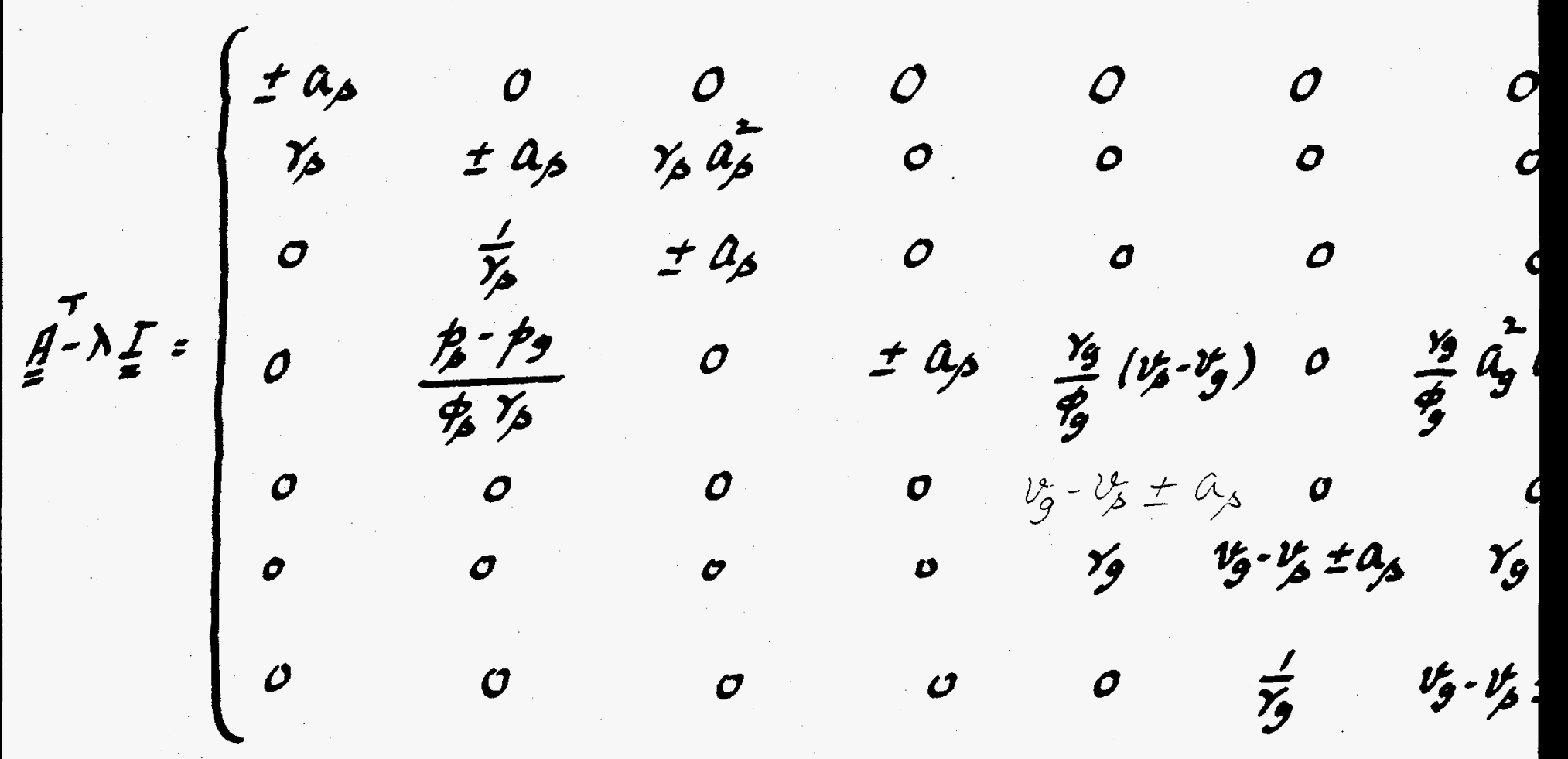


17

There are two possibilities:

(a). $v_{g}-v_{s} \pm a_{s} \neq 0 . \quad$ Then,

$$
\begin{aligned}
& l_{1}=0, l_{2} \pm r_{s} a_{s} l_{3}=0, l_{5}=l_{6}=l_{7}=0, \\
& \frac{p_{s}-p_{9}}{\phi_{s} r_{s}} l_{2} \pm a_{3} l_{4}=0 \\
& \therefore l=\left(0, \mp r_{p} a_{s} l_{3}, l_{3}, \frac{p_{s}-p_{g}}{\phi_{p}} l_{3}, 0,0,0\right)^{\top}
\end{aligned}
$$

Thus, $\lambda=v_{p}-a_{p} \Rightarrow$

$$
l=\left(0,-r_{p} a_{p}, 1, \frac{p_{p}-p_{g}}{\phi_{p}}, 0,0,0\right)^{\top}
$$

and $\lambda=v_{p}+a_{\beta} \Rightarrow$

$$
\left(0, r_{s} a_{p}, 1, \frac{p_{s}-p_{p}}{\phi_{p}}, 0,0,0\right)^{\top} \text {. }
$$

(b) If $v_{p}-v_{g}=a_{p}$ or $-a_{p}$, then, in addition to the eigenvector (1) or (2) found above, there is another one, given by

$$
l=\left(0,0,0,0,0,-a_{g}^{2}, 0,1\right)
$$

The reason for this additional eigenvector is that the eigenvalue $v_{p}+a_{s}\left(\alpha v_{p}-a_{\beta}\right)$ coincides wilt e the eigenvalue $v_{g}$. 
(iii) $\lambda=v_{g}$.

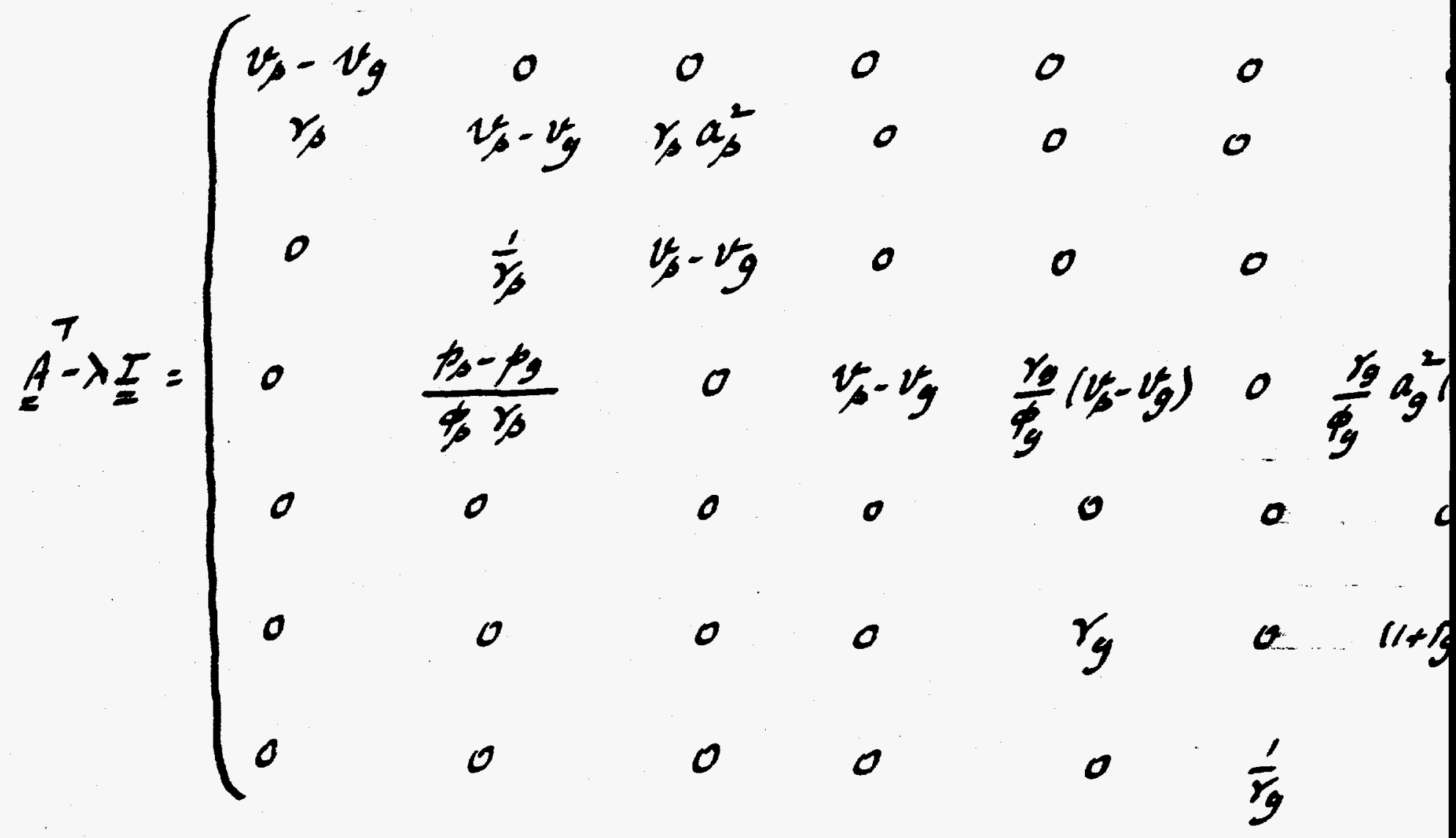

We shall assume $v_{p} \neq v_{g}$. (The case $v_{\%}=v_{g}$ has bo dealt wilt e)

$$
\begin{aligned}
& l_{1}=0=l_{2}=l_{3}=l_{6} \\
& l_{s}+a_{g}^{2} l_{7}=0 \\
& \left.l_{4}+\frac{r_{g}}{\phi_{g}} l_{5}+\frac{r_{g}}{\phi_{g}} a_{g}^{2} l_{7}=0\right\} \Rightarrow l_{4}=0, l_{5}=-a_{g}^{2} l_{1} \\
& \therefore \quad l=\left(\begin{array}{lllll}
0, & 0, & 0, & -a_{g}^{2}, 0,1
\end{array}\right) \text {. }
\end{aligned}
$$

(iv) $\quad t=v_{g} \mp a_{g}$ 
19.

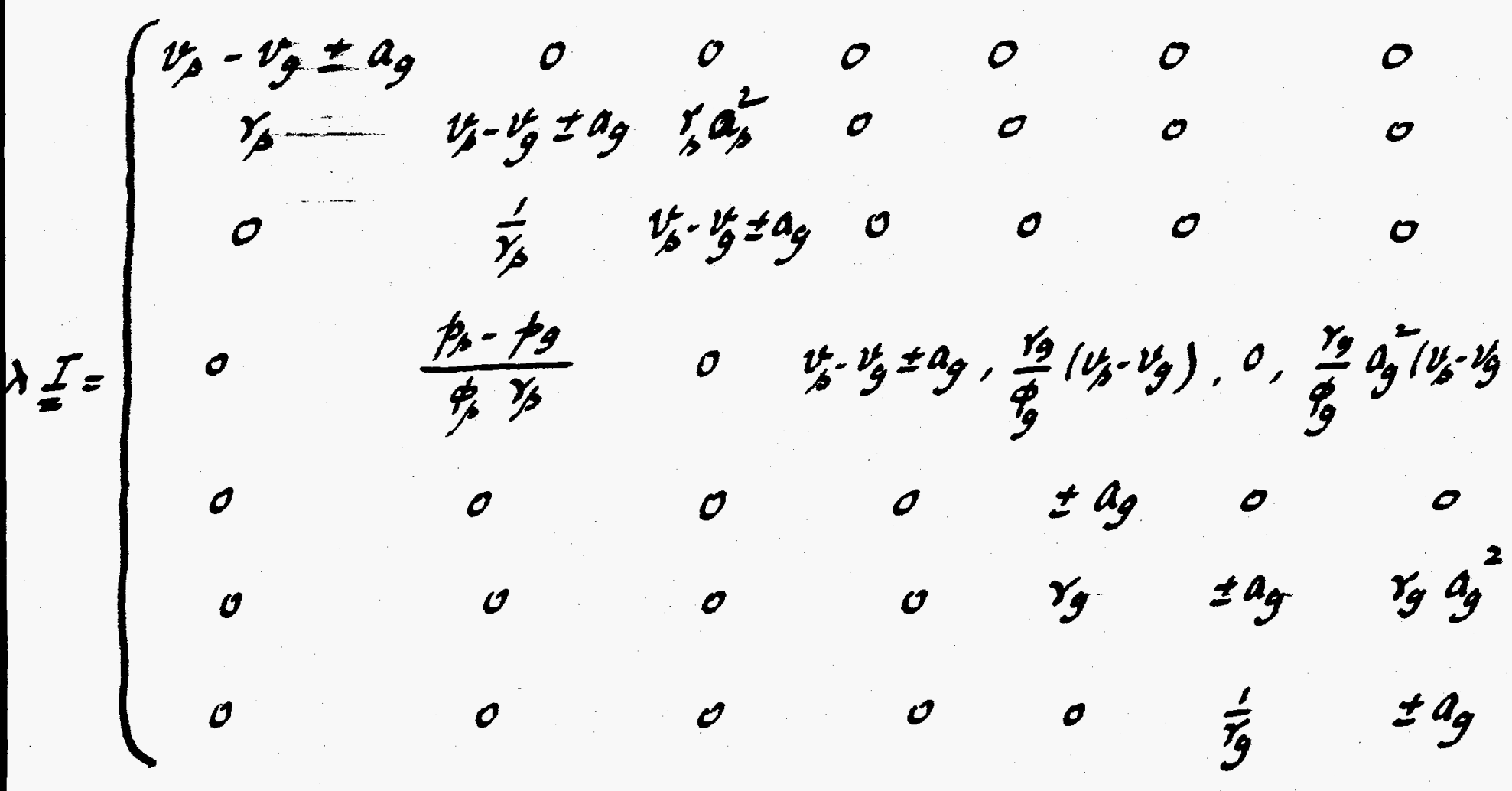

(a) $\quad V_{s}-V_{g} \pm a_{g} \neq 0$

$$
\begin{aligned}
& l_{1}=l_{2}=l_{3}=l_{5}=0 \\
& l_{6} \pm r_{g} a_{g} l_{7}=0 \\
& \left(v_{s}-v_{g} \pm a_{g}\right) l_{4}+\frac{r_{g}}{\phi_{g}} a_{g}^{2}\left(v_{g}-v_{g}\right)^{l}=0 \\
& \therefore l=\left\langle 0,0,0,-\frac{r_{g} a_{g}^{2}\left(v_{s}-v_{g}\right)}{q_{g}\left(v_{b}-v_{g}+a_{g}\right)}, 0, \mp r_{g} a_{g},\right\rangle .
\end{aligned}
$$

(b) $\quad v_{f}-v_{g} \pm a_{g}=0$. In this case,

$$
\underline{l}=\langle 0,0,0,1,0,0,0\rangle .
$$


Sun try:

Real that the eigenvalues are

$$
\begin{aligned}
& \lambda_{1}=\lambda_{1} \nu_{5}, \lambda_{3}=v_{5}+a_{5}, \lambda_{4}=v_{5}-\lambda_{5}, \lambda_{5}=v_{9}, \\
& \lambda_{6}=y_{g}+a_{g}, \lambda_{7}=v_{g}-a_{g} .
\end{aligned}
$$

supp. that there is no further an g left eigenvectors frame

$$
\begin{aligned}
& l, 1-a_{p}^{2}, 0,1,0,0, \\
& l_{2}=0,0,0,1,0 \text {, } \\
& l_{3}=0, r_{p} a_{p}, 1, \frac{p_{s}-p_{g}}{\phi_{p}}
\end{aligned}
$$

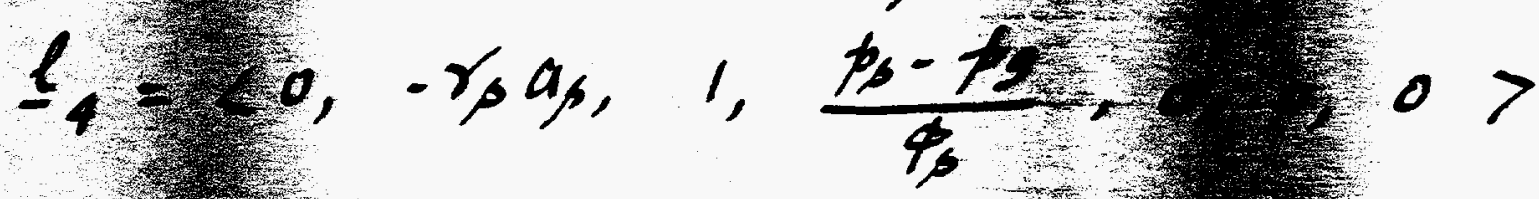

$$
\begin{aligned}
& \underline{l}_{5} \leqslant(0,0,0,0,-4,0,1> \\
& l_{6} v_{0}, 0,0,-\frac{r_{g} a_{g}^{2}\left(v_{s}-v_{g}\right.}{\phi_{g}\left(v_{p}-v_{g}-a_{g}\right)}, r_{g} a_{g},
\end{aligned}
$$

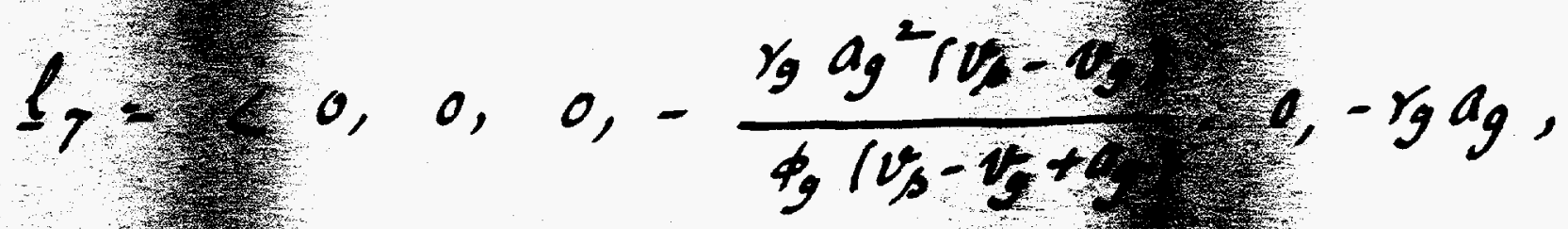

Now, the remains complete. It sf $\lambda_{3}=1, \lambda_{4}=\lambda_{5}$ or, $\lambda_{3} \propto \lambda_{3}$ ? of eigenura pet: 
The tration is different if 1 , if $t_{g}=v_{g} \pm a_{g}$. Ihen, eithere cons wilte $\underline{l}_{2}$ and completenew

Fer I moment, let us arsecme wet raut. Then, the equativis. char istec frum:

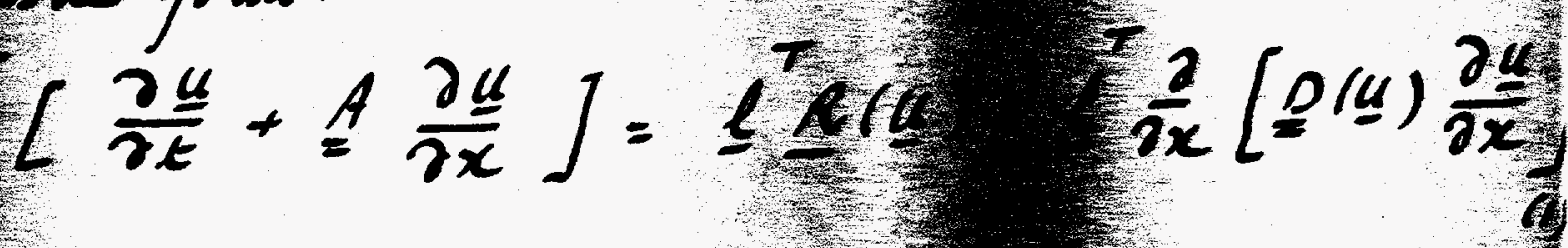

The 5 aled farme is:

$$
\begin{aligned}
& \frac{\partial p_{s}}{\partial t} \geqslant \frac{\partial p_{s}}{\partial x}-a_{s}^{2}\left(\frac{\partial r_{s}}{\partial t}+v_{s} \frac{\partial y_{s}}{\partial t}\right) \\
& +\frac{\pi}{\phi} \quad\left(\frac{\partial T_{0}}{\partial x}\right) \\
& \frac{\partial \phi}{\partial t}+\frac{\partial \phi}{\partial x}=R_{4} \\
& \frac{\partial p_{s}}{\partial t}+\left(a_{p}\right) \frac{\partial p_{s}}{\partial x} \pm r_{p} a_{p}\left[\frac{\partial v_{p}}{\partial t}\right. \\
& +\frac{t}{\phi_{s}}\left[\frac{\partial \phi_{s}}{\partial t}+\left(v_{s} \pm a_{\beta}\right)-\frac{\partial t}{\partial t}\right. \\
& \left.= \pm r_{p}\right) \tilde{R}_{2}+\mathcal{R}_{3}+\frac{\Gamma_{p}}{\phi_{b}} \frac{\partial}{\partial x}\left(\tilde{k}_{b} \frac{\partial T_{p}}{\partial x}\right) \\
& \frac{\partial p_{g}}{\partial t}+\frac{\partial p_{g}}{\partial x}-a_{g}^{2}\left(\frac{\partial y_{g}}{\partial t}+v_{g} \frac{\partial y_{g}}{\partial x}\right)=
\end{aligned}
$$




$$
\begin{aligned}
& \frac{\partial p_{g}}{\partial t}+\left(v_{g} \pm a_{g}\right) \frac{\partial p_{g}}{\partial x} \pm r_{g} a_{g}\left[\frac{\partial v_{g}}{\partial t}+\left(v_{g} \pm a_{g}\right) \frac{\partial v_{g}}{\partial x}\right] \\
& -\frac{\left.v_{g} a_{g}^{2} t_{g}-v_{g}\right)}{\phi_{g}\left(v_{s}-v_{g} a_{g}\right)}\left[\frac{\partial \phi_{f}}{\partial t}+\left(v_{g} \pm a_{g}\right) \frac{\partial \phi_{g}}{\partial x}\right] \\
& =-\frac{v_{g} a_{g}^{2}\left(v_{s}-v_{g}\right)}{\phi_{g}\left(v_{t}-v_{g} a_{g}\right)} R_{A} \pm y_{g} a_{g} R_{G}+R_{T}+\frac{\sigma_{g}}{g_{g}} \frac{\partial}{\partial x}\left(\tilde{k}_{g} \frac{\partial T}{\partial x}\right.
\end{aligned}
$$

Let us take a bock at the source terms.

$$
\begin{aligned}
& S_{1} \equiv R_{3}-a_{\beta}^{2} R_{1}=-\frac{T_{B}}{\phi_{\phi}} \tilde{h}\left(T_{p}-T_{g}\right)-\frac{F}{\phi_{\beta}}\left[r_{\beta} a_{A}^{2}=T_{p} p_{\beta}\right] \\
& +\frac{r_{s} a_{b}^{2}}{\phi_{s}} F \\
& =-\frac{\rho_{\beta}}{\phi_{s}} \tilde{L}\left(T_{p}-T_{g}\right)+\frac{\Gamma_{\beta} \beta_{s}}{\phi_{s}} F . \\
& s_{R} \equiv R_{4}=F_{+} \tilde{r} \Phi \\
& s_{3} \equiv \pm \alpha_{\beta} a_{\beta} R_{2}+R_{3}+\frac{p_{s}-p_{9}}{\phi_{0}} R_{4} \\
& =\mp r_{p} \alpha_{p}\left[\left(\tilde{\delta}+\frac{1}{2} \tilde{r} \gamma_{p} \Phi\right)\left(v_{p}-v_{g}\right)\right]-\frac{T_{\beta}}{\phi_{p}} \tilde{h}\left(T_{p}-T_{g}\right) \\
& -\frac{F}{\phi_{\beta}}\left(Y_{\beta} a_{\beta}^{2}-\rho_{\beta} \beta\right)+\frac{p_{\beta}-p_{g}}{\phi_{\beta}}(F+\tilde{F} \Phi) \text {. } \\
& s_{s} \equiv-a_{g}^{2} R_{s}+R_{7}=-a_{g}^{2}\left[\frac{y_{g}}{\phi_{g}} F-\left(1-\frac{r_{g}}{r_{s}}\right) \tilde{r} \gamma_{s} \Phi\right]
\end{aligned}
$$

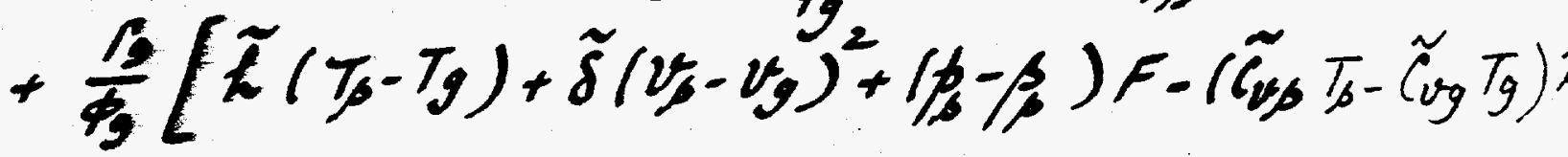


23

$$
\begin{aligned}
& +\frac{p_{g}}{\phi_{g}} F-\frac{1}{\phi_{g}} \Gamma_{g} \tilde{r_{x g}} T_{g}\left(1-\frac{r_{g}}{r_{s}}\right) \tilde{r} \gamma_{s} \Phi . \\
& s_{G} \equiv-\frac{r_{g} a_{g}^{2}\left(v_{s}-v_{g}\right)}{f_{g}\left(v_{s}-v_{g} a_{g}\right)} R_{4} \pm r_{g} a_{g} R_{b}+R_{7} \\
& =-\frac{v_{g} a_{g}^{2}\left(v_{p}-v_{g}\right)}{\phi_{g}\left(v_{p}-v_{g} F a_{g}\right)}(F+\tilde{r} \Phi) \pm \frac{a_{g}}{g g}\left(\tilde{\delta}-\frac{1}{2} \tilde{r} v_{p} \Phi\right)\left(v_{s}-v_{g}\right. \\
& +\frac{\Gamma_{g}}{\phi_{g}}\left[\tilde{R}\left(T_{p}-T_{g}\right)+\tilde{\delta}\left(v_{s}-v_{g}\right)^{2}+\left(p_{p}-\beta_{s}\right) F-\right.
\end{aligned}
$$

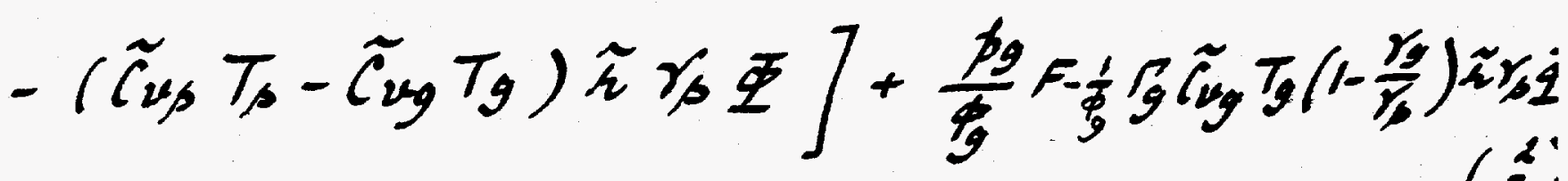


Equations in near-conserwation form.

Start with the dimensionless equs. on fo .10. Note the fallowing:

Mass

$$
\begin{aligned}
& \frac{\partial}{\partial t}\left(\phi_{p} \gamma_{p}\right)+\frac{\partial}{\partial x}\left(\phi_{s} \gamma_{p} v_{s}\right)
\end{aligned}
$$

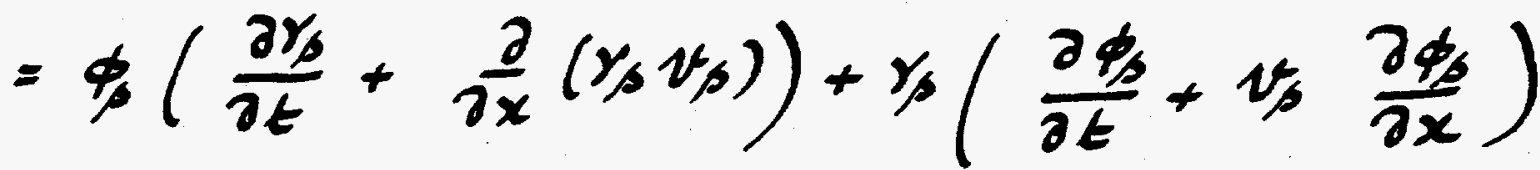

$$
\begin{aligned}
& =-y_{p} F+y_{f} f+y_{p} \tilde{r} \Phi
\end{aligned}
$$

Similarly,

$$
\begin{aligned}
& \frac{\partial}{\partial t}\left(\phi_{g} r_{g}\right)+\frac{\partial}{\partial x}\left(\phi_{g} r_{g} v_{g}\right) \\
& =\phi_{g}\left(\frac{\partial r_{g}}{\partial t}+\frac{\partial}{\partial x}\left(r_{g} v_{g}\right)\right)+r_{g}\left(\frac{\partial \phi_{g}}{\partial t}+v_{g} \frac{\partial \phi_{g}}{\partial x}\right) \\
& =r_{g} / r_{g}\left(v_{p}-r_{g}\right) \frac{\partial \phi_{g}}{\partial x}-\left(1-\frac{r_{g}}{r_{p}}\right) \tilde{r} r_{p} \Phi \\
& -r_{g}\left[\left(v_{g}-v_{p}\right) \frac{\partial \phi_{p}}{\partial x}+\phi+\tilde{g} \bar{\Phi}\right]
\end{aligned}
$$

Momentum

$$
\begin{aligned}
& \frac{\partial}{\partial t}\left(\phi_{\beta} Y_{\beta} v_{\beta}\right)+\frac{\partial}{\partial x}\left[\phi_{\beta} y_{\beta} v_{p}^{2}+\phi_{\beta} p_{\beta}\right] \\
& =v_{\beta}\left[\frac{\partial}{\partial t}\left(\phi_{\beta} \gamma_{s}\right)+\frac{\partial}{\partial x}\left(\phi_{\beta} \gamma_{\beta} v_{\beta}\right)\right] \\
& +\phi_{p} \gamma_{\beta}\left[\frac{\partial v_{s}}{\partial t}+v_{p} \frac{\partial v_{s}}{\partial x}\right]+\phi_{\beta} \frac{\partial p_{p}}{\partial x}+p_{\beta} \frac{\partial \phi_{s}}{\partial x}
\end{aligned}
$$


25.

$$
\begin{aligned}
= & v_{p} r_{p} \tilde{r} \Phi-\phi_{p} \frac{\partial p_{p}}{\partial x}+\left(p_{g}-p_{p}\right) \frac{\partial \phi_{p}}{\partial x} \\
& -\left(\tilde{\delta}+\frac{1}{2} \tilde{r} v_{p} \Phi\right)\left(v_{p}-v_{g}\right)+\phi_{p} \frac{\partial \phi_{x}}{\partial x}+p_{p} \frac{\partial \phi_{p}}{\partial x} \\
= & p_{g} \frac{\partial \phi_{p}}{\partial x}+v_{p} \tilde{r} \gamma_{p} \Phi-\left(\tilde{\delta}+\frac{1}{2} \tilde{r} r_{p} \Phi\right)\left(v_{p}-v_{g}\right) .
\end{aligned}
$$

similurly,

$$
\begin{aligned}
& \frac{\partial}{\partial t}\left(\phi_{g} r_{g} v_{g}\right)+\frac{\partial}{\partial x}\left[\phi_{g} \gamma_{g} v_{g}^{2}+\phi_{g} p_{g}\right] \\
& =v_{g}\left[\frac{\partial}{\partial t}\left(\phi_{g} \gamma_{g}\right)+\frac{\partial}{\partial x}\left(\phi_{g} \gamma_{g} v_{g}\right)\right] \\
& +\phi_{g} V_{g}\left[\frac{\partial v_{g}}{\partial t}+v_{g} \frac{\partial v_{g}}{\partial x}\right]+\phi_{g} \frac{\partial p_{g}}{\partial x}+p_{g} \frac{\partial \phi_{g}}{\partial x} \\
& =-v_{g} \tilde{r} r_{\beta} \Phi-\phi_{g} \frac{\partial p_{g}}{\partial x}+\left(\tilde{\delta}-\frac{1}{2} \tilde{i} \gamma_{p} \Phi\right)\left(v_{p}-v_{g}\right) \\
& +\phi_{g} \frac{\partial p_{g}}{\partial x}+\lg \frac{\partial \phi_{g}}{\partial x} \\
& =-v_{s} \tilde{r} \gamma_{s} \Phi+\left(\tilde{\delta}+\frac{1}{2} \tilde{\kappa} \gamma_{p} \Phi\right)\left(v_{p}-v_{g}\right)+p_{g} \frac{\partial \Phi_{g}}{\partial x} .
\end{aligned}
$$

Enagy

$$
E_{a}=e_{a}+\frac{1}{2} v_{a}^{2} \quad \text { (dimensional) }
$$

In dimersionless ferm, $F_{a}=\tilde{c}_{v_{a}} T_{a}+\frac{1}{2} v_{a}^{2}$. Now, $\frac{\partial}{\partial t}\left(\phi_{p} \gamma_{p} F_{p}\right)+\frac{\partial}{\partial x}\left[\phi_{p} \gamma_{p} E_{p} v_{p}+\phi_{p} p_{p} v_{p}\right]$ 


$$
\begin{aligned}
& =\frac{\partial}{\partial t}\left(\phi_{\beta} \gamma_{\rho} e_{\beta}+\phi_{\beta} y_{\rho} \frac{1}{\alpha} v_{p}^{2}\right)
\end{aligned}
$$

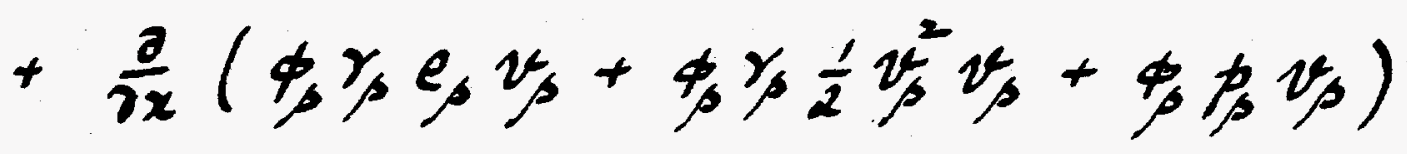

$$
\begin{aligned}
& =\left(e_{p}+\frac{1}{2} v_{p}^{2}\right)\left[\frac{\partial}{\partial t}\left(\phi_{p} v_{p}\right)+\frac{\partial}{\partial x}\left(\phi_{p} v_{p} v_{p}\right)\right] \\
& +\phi_{s} v_{p}\left[\frac{\partial e_{s}}{\partial t}+v_{s} \frac{\partial e_{s}}{\partial x}\right]+\phi_{s} v_{s} v_{s}\left[\frac{\partial v_{s}}{\partial t}+v_{s} \frac{\partial v_{s}}{\partial x}\right] \\
& +\frac{\partial}{\partial x}\left(\phi_{p} p_{s} v_{p}\right)
\end{aligned}
$$

On using the apposperiate expressions from pages 10 and

$$
\begin{aligned}
& =\left(e_{p}+\frac{1}{2} v_{p}^{2}\right) v_{p} \tilde{r} \Phi-\phi_{p} p_{p} \frac{\partial v_{p}}{\partial x}+\frac{\partial}{\partial x}\left(\tilde{k}_{p} \frac{\partial T_{p}}{\partial x}\right) \\
& -\tilde{k}\left(T_{p}-T_{g}\right)-\left(p_{p}-\beta_{p}\right) F+v_{p}\left[-\phi_{p} \frac{\partial p_{p}}{\partial x}+\left(p_{p}-p_{p}\right) \frac{\partial \phi_{p}}{\partial x}\right. \\
& \left.-\left(\tilde{\delta}+\frac{1}{2} \tilde{r} x_{\bar{q}}\right)\left(v_{p}-v_{g}\right)\right]+\frac{\partial}{\partial x}\left(\phi_{p} p_{p} v_{p}\right) .
\end{aligned}
$$

Finally, as above,

$$
\begin{aligned}
& \frac{\partial}{\partial t}\left(\phi_{g} V_{g} F_{g}\right)+\frac{\partial}{\partial x}\left[g_{g} r_{g} F_{g} v_{g}+\phi_{g} p_{g} v_{g}\right] \\
& =\frac{\partial}{\partial t}\left(\phi_{g} y_{g} e_{g}+\phi_{g} y_{g} \frac{1}{2} v_{g}^{2}\right)+\frac{\partial}{\partial x}\left[\phi_{g} r_{g} e_{g} v_{g}+\phi_{g} y_{g} \frac{1}{2} v_{g}^{2} ?\right. \\
& \left.+\phi_{g} v_{g}\right] \\
& =\left(g_{g}+\frac{1}{2} v_{g}^{2}\right)\left[-v_{p} \tilde{r} \Phi\right]-\phi_{g} p_{g} \frac{\partial v_{g}}{\partial x}+\frac{\partial}{\partial x}\left(\tilde{k_{g}} \frac{\partial T}{\partial x}\right)
\end{aligned}
$$


25

Summany: near-conservation form of equations

$$
\begin{aligned}
& \frac{\partial}{\partial t}\left(\phi_{p} y_{p}\right)+\frac{\partial}{\partial x}\left(\phi_{p} y_{p} v_{p}\right)=C_{s}^{*} \\
& \frac{\partial}{\partial t}\left(\phi_{g} Y_{y}\right)+\frac{\partial}{\partial x}\left(\phi_{g} r_{g} v_{g}\right)=-C_{g}^{*} \\
& \frac{\partial}{\partial t}\left(\phi_{p} \gamma_{s} v_{p}\right)+\frac{\partial}{\partial x}\left[\phi_{p} \gamma_{s} v_{p}^{2}+\phi_{p} p_{p}\right]-p_{\rho} \frac{\partial \phi_{p}}{\partial x}=m_{p}^{*} \\
& \frac{\partial}{\partial t}\left(\phi_{g} v_{g} v_{g}\right)+\frac{\partial}{\partial x}\left[\phi_{g} v_{g}^{2} y_{g}+\phi_{g} \phi_{g}\right]-t_{g} \frac{\partial \phi_{g}}{\partial x}=-m_{A}^{*} \\
& -\phi_{s} \frac{\partial \phi_{s}}{\partial x} v_{s}
\end{aligned}
$$

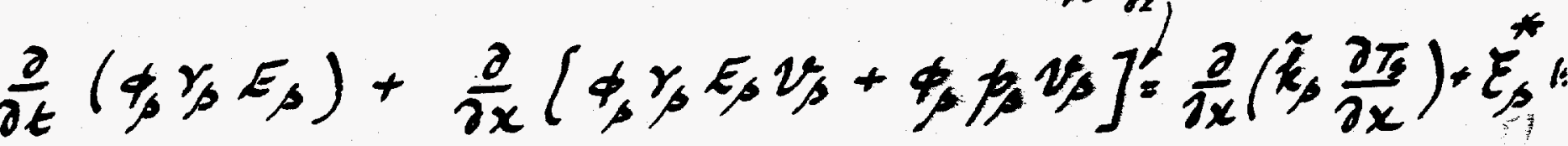

$$
\begin{aligned}
& \frac{\partial}{\partial t}\left(\phi_{g} r_{g} F_{g}\right)+\frac{\partial}{\partial x}\left[\phi_{g} r_{g} F_{g} v_{g}+\phi_{g} F_{g} v_{g}\right]_{g}=\frac{\partial}{\partial x}\left(\tilde{k}_{g} \frac{\partial T_{g}}{\partial x}\right)-\xi_{s} \\
& -\operatorname{pg} \frac{\partial \phi}{\partial x} v_{s}
\end{aligned}
$$

Here,

$$
\begin{aligned}
& C_{\beta}^{*}=\tilde{r} \gamma_{\rho} \Phi \\
& m_{\beta}^{*}=v_{\beta} \mathcal{C}_{\beta}^{*}-\left(\tilde{s}+\frac{1}{2} \mathcal{C}_{\beta}^{*}\right)\left(v_{\beta}-v_{g}\right) \\
& \xi_{s}^{*}=E_{\beta} C_{s}^{*}-\tilde{k}\left(T_{p}-T_{g}\right)-\left(F_{p}-\beta_{p}\right) F=\left(\tilde{\delta}+\frac{1}{2} C_{\beta}^{*}\right)\left(v_{p}-v_{g},\right. \\
& E_{a}=\tilde{C}_{v a} T_{a}+\frac{1}{2} v_{a}^{2} ; e_{a}=\tilde{C}_{v a} T_{a} .
\end{aligned}
$$

Hise, $\quad \frac{\partial Y_{s}}{\partial t}+\frac{\partial}{\partial x}\left(Y_{s} V_{s}\right)=-\frac{Y_{s}}{F_{s}} F_{0}$

(II) 


$$
\begin{aligned}
& +\tilde{h}\left(T_{p}-T_{g}\right)+\left(p_{p}-p_{p}\right) F-\left(v_{p}-v_{g}\right) p_{g} \frac{\partial \phi_{p}}{\partial x} \\
& +\tilde{s}\left(v_{p}-v_{g}\right)^{2}-\left(e_{p}-\varepsilon_{q}\right) \tilde{r} \gamma_{p} \Phi+v_{g}\left[-\phi_{p} \frac{\partial p_{g}}{\partial x}\right. \\
& \left.+\left(\tilde{\delta}-\frac{1}{2} \tilde{r} y_{p} \Phi\right)\left(v_{p}-v_{g}\right)\right]+\frac{\partial}{\partial x}\left(\phi_{g} p_{g} v_{g}\right) \\
& =\frac{\partial}{\partial x}\left(\tilde{k}_{g} \frac{\partial T_{g}}{\partial x}\right)+\tilde{k}\left(T_{p}-T_{g}\right)+\left(p_{s}-\beta_{p}\right) F+\nu_{p} p_{g} \frac{\partial}{\partial} \\
& +\left\{\tilde{\delta}\left(v_{p}-v_{g}\right)^{2}+v_{g}\left(\tilde{s}-\frac{1}{2} \tilde{z} \gamma_{s} \Phi\right)\left(v_{s}-v_{g}\right)\right. \\
& \left.-\frac{1}{2} v_{g}^{2} r_{p} \tilde{r} \Phi\right\}-e_{p} \tilde{r} r_{p} \Phi
\end{aligned}
$$

The three terms, can be combined to yield $-\frac{1}{2} v_{\beta}^{2} r_{\beta} \tilde{r} \Phi+v_{p}\left(\tilde{\delta}+\frac{1}{2} \tilde{r} r_{p} \Phi\right)\left(v_{p}-v_{g}\right)$. 
ITEM 2 
1.

On the Solid Equation of State.

B-N (1956, p.871, eq. (61)) start with the Helmhalte fue-encyyy function $\psi$, in the following dimensional forme:

$$
\begin{aligned}
& \psi_{p}\left(Y_{p}, T_{p}\right)=c_{\nu}^{b}\left(T_{p}-T_{0}\right)+c_{\mu}^{b}\left(T_{p}-T_{0}\right) \Gamma_{p}\left(\frac{Y_{p}}{Y_{p 0}}-1\right) \\
& -c_{\nu}^{\infty} T_{p} \ln \frac{T_{p}}{T_{p}^{0}}+\frac{1}{r_{p}^{0}} \frac{K_{T}}{N(N-1)}\left[\left(\frac{r_{0}}{r_{p 0}}\right)^{N-1}-(N-1)\left(1-\frac{r_{s 0}}{r_{p}}\right)-1\right] .
\end{aligned}
$$

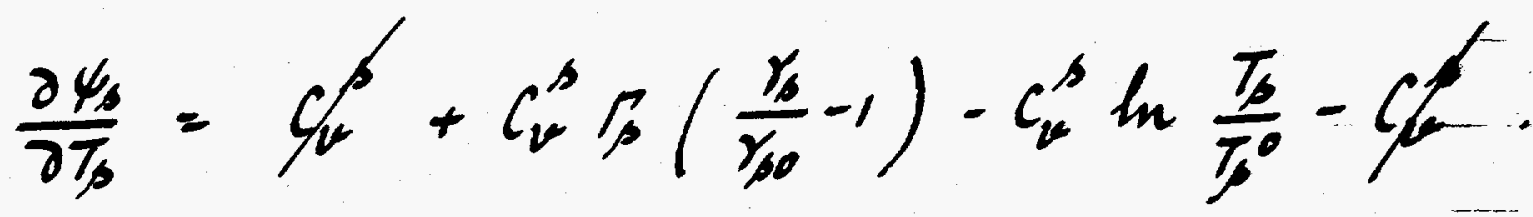

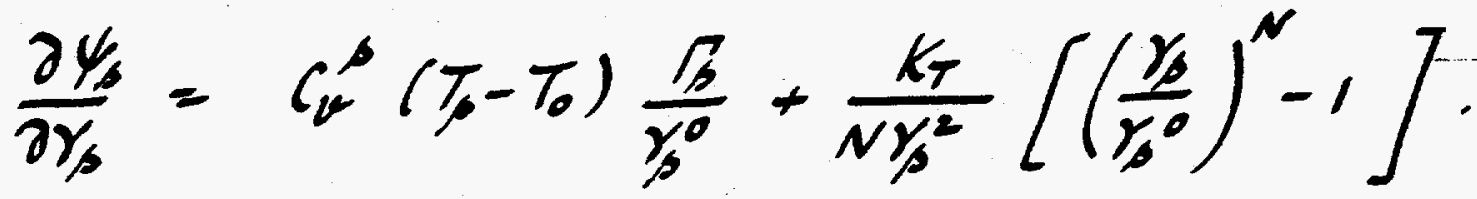

Now,

$$
\begin{aligned}
& p_{p}=r_{p}^{2} \frac{\partial \psi_{s}}{\partial r_{p}}=r_{p}^{2} c_{\nu}^{s}\left(T_{p}-T_{0}\right) \frac{\Gamma_{s}}{r_{p}^{0}}+\frac{K_{T}}{N}\left[\left(\frac{r_{p}}{r_{p}^{0}}\right)^{N}-1\right] . \\
& e_{p}=\psi_{s}-T_{p} \frac{\partial \psi_{s}}{\partial T_{p}}=c_{\mu}^{b}\left(T_{p}-T_{0}\right)-c_{\nu}^{s} T_{0} \Gamma_{p}\left(\frac{r_{s}}{r_{s 0}}-1\right) \\
& +\frac{1}{r_{s}^{0}} \frac{K_{T}}{N(N-1)}\left[\left(\frac{Y_{s}}{r_{s 0}}\right)^{N-1}-(N-1)\left(1-\frac{Y_{s 0}}{Y_{p}}\right)-1\right] \text {. }
\end{aligned}
$$

Eliminativg To from (4) and (5),

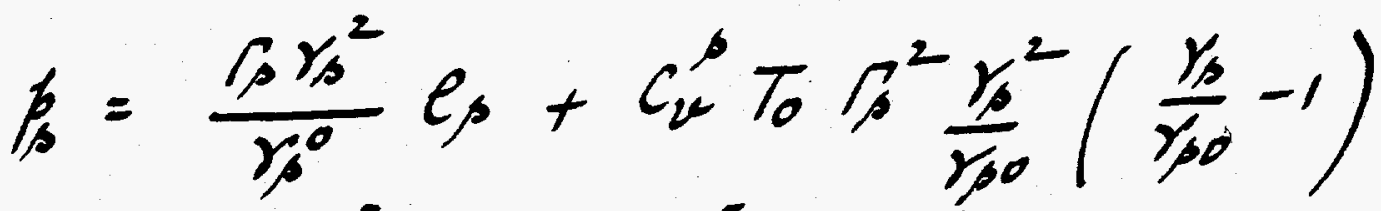

$$
\begin{aligned}
& -\frac{\Gamma_{p} Y_{s}^{2}}{Y_{p 0}^{2}} \frac{K_{T}}{N(N-1)}\left[\left(\frac{Y_{s}}{Y_{s}^{0}}\right)^{N-1}-(N-1)\left(1-\frac{Y_{s 0}}{Y_{p}}\right)-1\right]+\frac{K_{T}}{N}\left[\left(\frac{Y_{s}}{Y_{p 0}}\right)^{N}-1\right] .
\end{aligned}
$$

(6) 
The lust result can be rewritten as

$$
\begin{aligned}
p_{s} & =r_{p} r_{s}\left(\frac{r_{s}}{r_{s}^{0}}\right)\left[e_{p}+\Gamma_{p} c_{\nu}^{s} T_{s}^{0}\left(\frac{r_{s}}{r_{s 0}}-1\right)\right. \\
& \left.-\frac{1}{r_{s 0}} \frac{K_{T}}{N(N-1)}\left\{\left(\frac{r_{s}}{r_{s 0}}\right)^{N-1}-(N-1)\left(1-\frac{r_{s 0}}{r_{s}}\right)-1\right\}\right] \\
& +\frac{K_{T}}{N}\left[\left(\frac{r_{s}}{r_{s 0}}\right)^{N}-1\right] .
\end{aligned}
$$

This expression is precisely the same as in Back (1985) p. 35, eq. (7)), if the factor $\left(\frac{Y_{s}}{Y_{0}}\right)$ outside the. square brackets is replaced by $1 .\left(\frac{y_{0}}{Y_{0}}\right)$ arts ide In fact, unless the above modification is made, the aba. equation fails to yield

$$
r_{s}=\frac{1}{r_{p}}\left(\frac{\partial p_{s}}{\partial e_{p}}\right)_{r_{p}}
$$

with the modification, consistency requires that (4) be changed

$$
p_{p}=T_{p} r_{p} c_{\nu}^{p}\left(T_{p}-T_{0 p}\right)+\frac{K_{T}}{N}\left[\left(\frac{T_{p}}{T_{p_{0}}}\right)^{N}-1\right] .
$$

Eq. (5) remains unaltered, while (I) $\Rightarrow$

$$
\begin{aligned}
& p_{p}=r_{p} r_{p}\left[e_{p}+C_{p} c_{\alpha}^{s} T_{p}^{0}\left(\frac{r_{p}}{r_{p}^{0}}-1\right)-\frac{1}{r_{p o}} \frac{K_{T}}{N(N-1)}\left\{\left(\frac{r_{p}}{r_{p}^{0}}\right)^{N-1}-\right.\right. \\
& \left.\left.-(N-1)\left(1-\frac{Y_{s 0}}{Y_{s}}\right)-1\right\}\right]+\frac{K_{T}}{N}\left[\left(\frac{Y_{s}}{Y_{s 0}}\right)^{N}-1\right] \text {. }
\end{aligned}
$$


3.

Let us define

$$
\begin{gathered}
\frac{K_{T}}{N(N-1)}\left\{\sigma^{N-1}-(N-1)\left(1-\frac{1}{\sigma}\right)-1\right\}=g(\sigma) \\
\quad \frac{K_{T}}{N}\left[\sigma^{N}-1\right]=L(\sigma) .
\end{gathered}
$$

Note that $g^{\prime}(\sigma)=\frac{h(\sigma)}{\sigma^{2}}, h^{\prime}(\sigma)=k_{z} \sigma^{N-1}$.

Then,

and

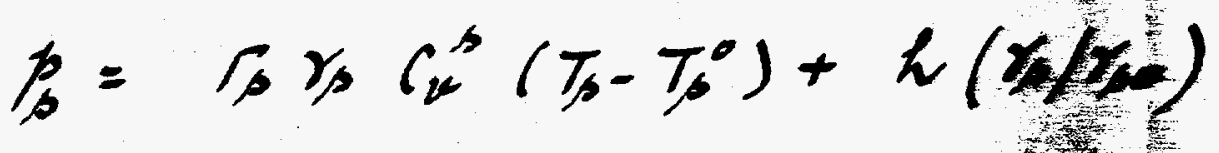

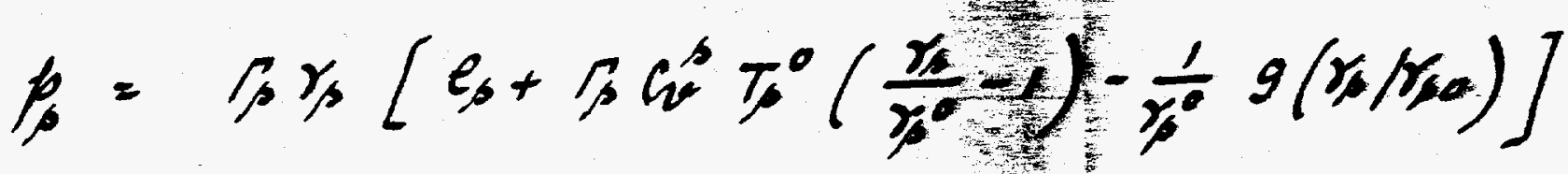

$$
\begin{aligned}
& +h\left(r_{p} / \gamma_{p o}\right) \text {. }
\end{aligned}
$$

From (13),

$$
\begin{aligned}
& \left.\frac{\partial p_{s}}{\partial r_{s}}\right)_{e_{p}}=r_{p}\left[\frac{p_{p}-h}{r_{p} r_{p}}\right]+\frac{1}{r_{p o}} h^{\prime}\left(\frac{r_{0}}{r_{p o}}\right)
\end{aligned}
$$

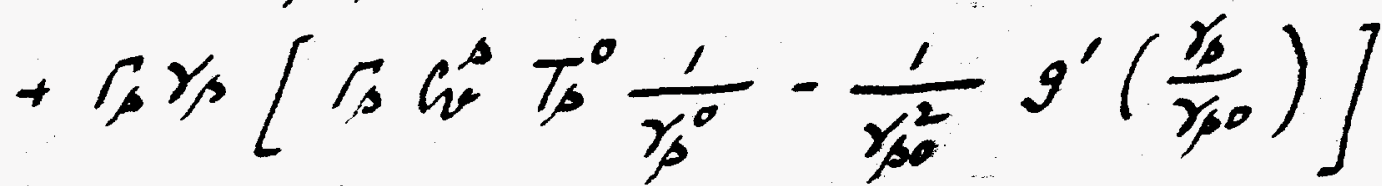

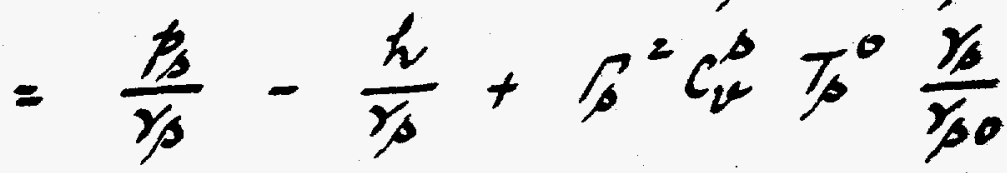

$$
\begin{aligned}
& +1 k^{\prime}\left(\frac{r_{0}}{0}\right)-r_{0} \frac{r_{0}}{1} g^{\prime}\left(\frac{r_{0}}{r_{0}}\right)
\end{aligned}
$$


On using (14),

$$
\begin{aligned}
& a_{s}^{2}=\frac{\left(\Gamma_{s}+1\right) p_{s}}{r_{p}}+{\beta_{p}^{2}}^{2} c_{\nu}^{\infty} T_{p}^{0} \frac{\gamma_{p}}{\gamma_{p}^{0}} \\
& +\frac{1}{r_{p o}}\left[h^{\prime}\left(\frac{r_{p}}{r_{p o}}\right)-r_{p} \frac{r_{p}}{r_{p o}} g^{\prime}\left(\frac{r_{p}}{r_{p o}}\right)\right]
\end{aligned}
$$

a

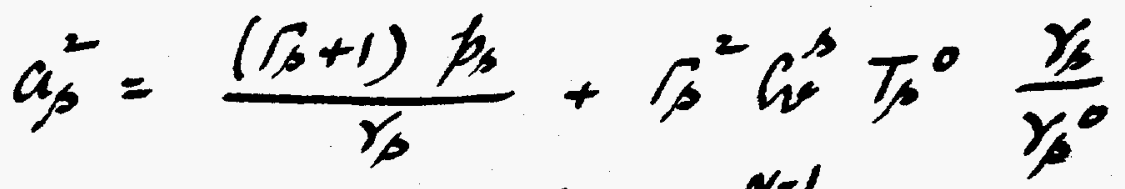

$$
\begin{aligned}
& +\frac{K_{T}}{Y_{s o}}\left[\left(\frac{y_{s}}{y_{p o}}\right)^{N-1}-r_{\beta} \frac{Y_{s o}}{y_{p}} \frac{1}{N}\left\{\left(\frac{y_{p}}{y_{p o}}\right)^{N}-1\right\}\right]
\end{aligned}
$$

$\alpha$

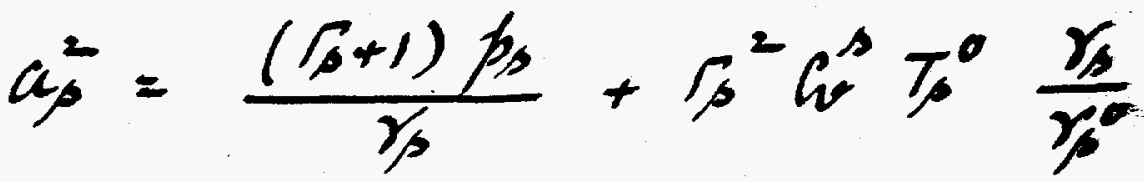

$$
\begin{aligned}
& +\frac{K_{T}}{r_{\beta}}\left[\left(\frac{Y_{b}}{Y_{00}}\right)^{N}\left(1-\frac{\Gamma_{\beta}}{N}\right)+\frac{\Gamma_{\beta}}{N}\right] .
\end{aligned}
$$


3.

Let us define

$$
\begin{gathered}
\frac{K_{T}}{N(N-1)}\left\{\sigma^{N-1}-(N-1)\left(1-\frac{1}{\sigma}\right)-1\right\}=g(\sigma) \\
\frac{K_{T}}{N}\left[\sigma^{N}-1\right]=h(\sigma) .
\end{gathered}
$$

Note that $g^{\prime}(\sigma)=\frac{h(\sigma)}{\sigma^{2}}, h^{\prime}(\sigma)=k_{z} \sigma^{N-1}$.

Then,

and

$$
\begin{aligned}
& p_{p}=r_{p} r_{p} c_{\nu}^{s}\left(T_{p}-T_{p}^{0}\right)+h\left(x / p_{p}\right)
\end{aligned}
$$

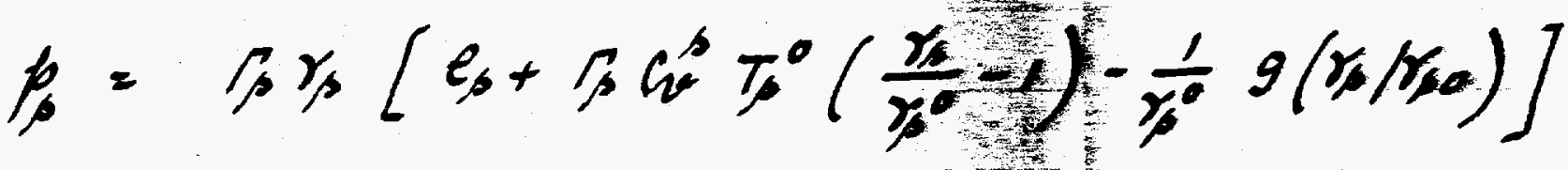

$$
\begin{aligned}
& +h\left(r_{p} / r_{p o}\right) \text {. }
\end{aligned}
$$

From (13),

$$
\begin{aligned}
& \left.\frac{\partial p_{p}}{\partial r_{s}}\right)_{e_{\beta}}=r_{\beta}\left[\frac{p_{p}-h}{r_{\beta} r_{p}}\right]+\frac{1}{r_{p o}} h^{\prime}\left(\frac{r_{p}}{r_{p o}}\right)
\end{aligned}
$$

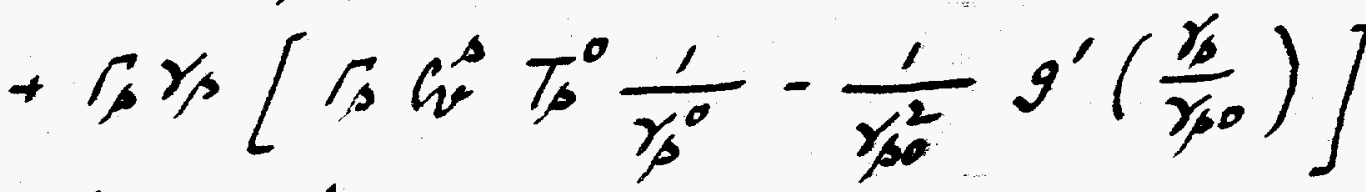

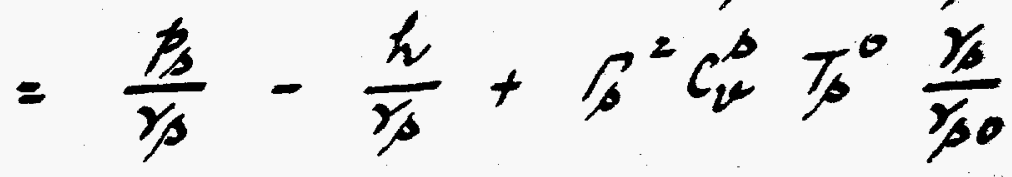

$$
\begin{aligned}
& +\frac{1}{r_{p o}} k^{\prime}\left(\frac{r_{0}}{r_{p 0}}\right)-r_{\beta} \frac{r_{s}}{r_{p o}} \frac{1}{r_{p o}} g^{\prime}\left(\frac{r_{0}}{r_{p o}}\right)
\end{aligned}
$$

Now, sound speed is given by

$$
\left.a_{\beta}^{2}=\frac{\partial p_{s}}{\partial r_{\beta}}\right)_{e_{s}}+\frac{\Gamma_{\beta} p_{s}}{r_{\beta}} .
$$




$$
\begin{aligned}
& {\left[\frac{N}{V}+\left(\frac{N}{y}-1\right)_{N}\left(\frac{\Delta \mathscr{X}}{\mathscr{X}}\right)\right] \frac{\mathscr{U}}{2 y}+}
\end{aligned}
$$

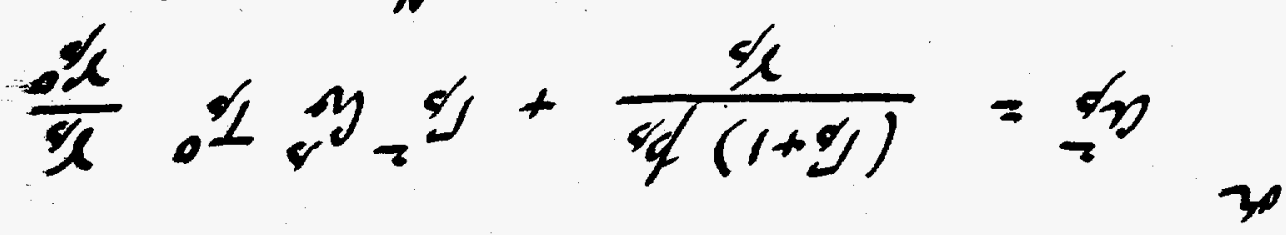

$$
\begin{aligned}
& {\left[\left\{1-\left(\frac{d x}{x}\right)\right] \div \frac{x}{d x} \dot{y}-\left(\frac{d x}{x}\right)\right] \frac{\Delta x}{-y}+} \\
& \frac{d x}{x} 0 y_{y} y_{2} y+\frac{y}{y(1+y)}=4 p
\end{aligned}
$$

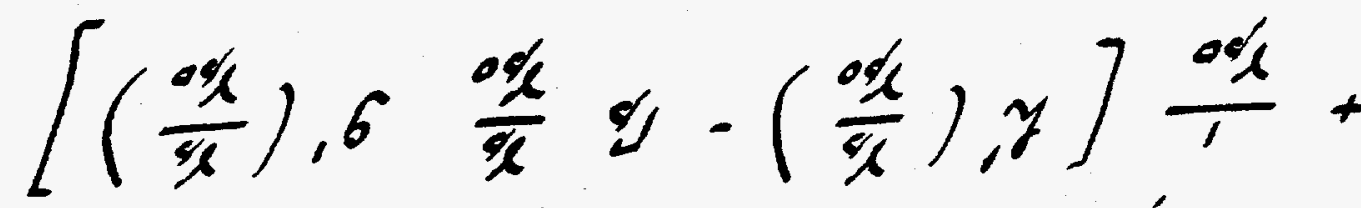

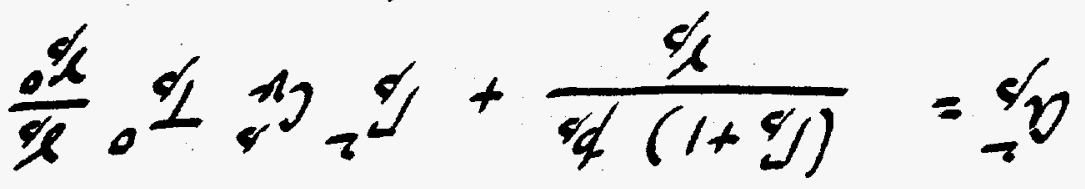

(b) finsn no 
5.

A simplified nonideal equation of state.

Let us assume that

$$
\begin{aligned}
& p_{p}=\Gamma_{p} r_{p} c_{\nu}^{s} T_{p}+h\left(\frac{r_{s}}{r_{p o}}\right) \\
& e_{p}=c_{\nu}^{s} T_{p}+\frac{1}{r_{p o}} g\left(\frac{r_{p}}{r_{p o}}\right)
\end{aligned}
$$

let us see if these equations are consistent wilt e a Helmbalte free-eneyy function.

Define

$$
\psi_{0}=c_{v}^{s} T_{p}\left[T_{p} \ln \gamma_{p}-\ln T_{\beta}\right]+\frac{1}{r_{p o}} g\left(\frac{Y_{p}}{r_{p o}}\right)
$$

(19)

Thess,

$$
\frac{\partial \psi_{s}}{\partial r_{\beta}}=c_{\nu}^{s} T_{\beta} \Gamma_{\beta} \frac{1}{r_{p}}+\frac{1}{r_{p o}^{2}} g^{\prime}\left(\frac{r_{p}}{r_{p o}}\right)
$$

and

$$
\begin{aligned}
p_{p}=r_{p}^{2} \frac{\partial \psi_{s}}{\partial r_{p}} & =c_{\nu}^{b} T_{p} \Gamma_{p} r_{p}+\frac{r_{p}^{2}}{r_{p o}^{2}} g^{\prime}\left(\frac{r_{p}}{r_{p 0}}\right) \\
& =c_{\nu}^{b} T_{p} \Gamma_{p} r_{p}+\hbar\left(\frac{r_{s}}{r_{p 0}}\right) \quad \text { oh }
\end{aligned}
$$

assuming $\quad h(\sigma)=\sigma^{2} g^{\prime}(\sigma)$.

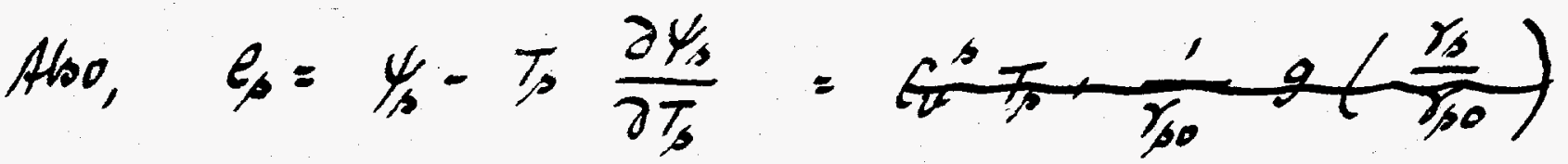

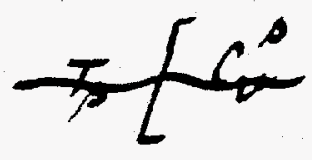

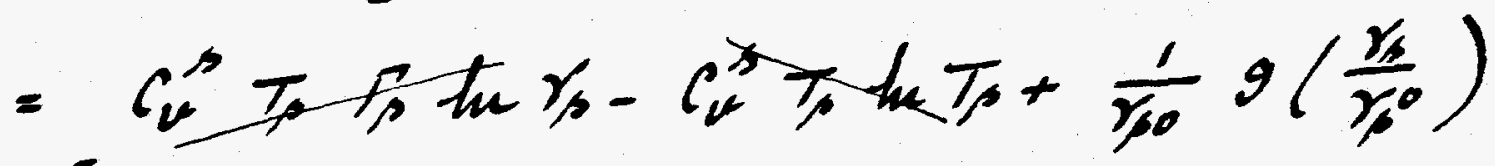

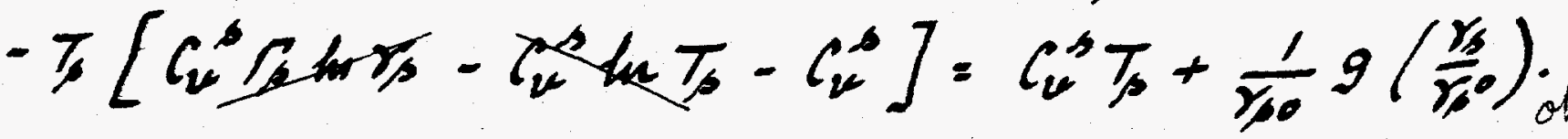


7.

Characteristic analysis revisited in view of more general equations of state.

Consider the dimensional equs. of state

$$
\begin{aligned}
& p_{a}=\Gamma_{a} r_{a} c_{v a} T_{a}+h_{a}\left(\frac{r_{a}}{r_{a}^{0}}\right) \\
& e_{a}=c_{v a} T_{a}+\frac{1}{r_{a}^{0}} g_{a}\left(\frac{r_{a}}{r_{a}^{0}}\right)
\end{aligned}
$$

Noudimensionalize:

units are $\bar{p}$ for $p_{a}, \bar{T}$ far $T_{a}, \bar{\gamma}$ for $r_{a}$. $\bar{p} / \bar{r}$ for $e_{a}, \bar{p} / \bar{r} \bar{\gamma}$ for c ra. Then,

$$
\begin{aligned}
& p_{a}=\Gamma_{a} r_{a} \tilde{C}_{v a} T_{a}+H_{a}\left(r_{a} / \gamma_{a}^{0}\right) \\
& e_{a}=\tilde{C_{v a}} T_{a}+\frac{1}{r_{a}^{0}} G_{a}\left(r_{a} / r_{a}^{0}\right)
\end{aligned}
$$

where $H_{a}=\hbar / \bar{p}, \quad G_{a}=\mathcal{g} / \bar{p} \cdot{ }_{(b)}\left[A\right.$ so, $a_{s}^{2}=\frac{\Gamma_{s}+1}{\gamma_{s}}\left(p_{s}-H_{s}\right)$ we also have

$$
H_{a}(\sigma)=\sigma^{2} G_{a}^{\prime}(\sigma) \text {. }
$$

Then,

$$
\begin{aligned}
\dot{p_{a}} & =\Gamma_{a} r_{a} \tilde{c}_{v a} \dot{T}_{a}+\Gamma_{a} \tilde{c_{v a}} T_{a} \dot{r}_{a}+\frac{1}{r_{a}^{0}} H_{a}^{\prime} \dot{r_{a}} \\
& =\Gamma_{a} r_{a} \tilde{c_{v a}} \dot{T_{a}}+\left[\Gamma_{a} \tilde{c_{v a}} T_{a}+\frac{1}{r_{a}^{0}} H_{a}^{\prime}\right] \dot{r}_{a}
\end{aligned}
$$

Ales, one may wite

$$
p_{a}=\Gamma_{a} r_{a}\left[e_{a}-\frac{1}{r_{a}^{0}} G_{a}\right]+H_{a}
$$




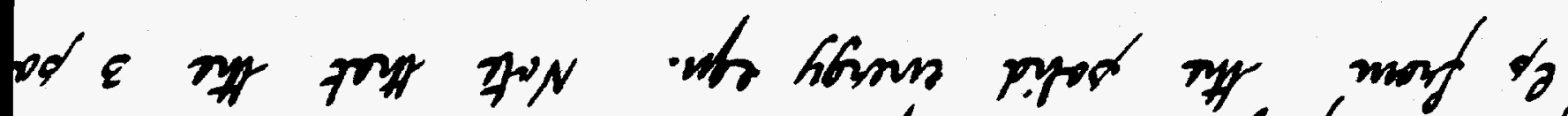

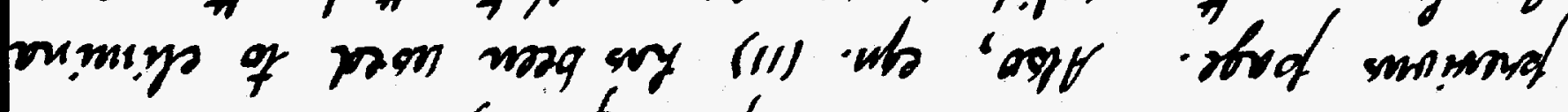

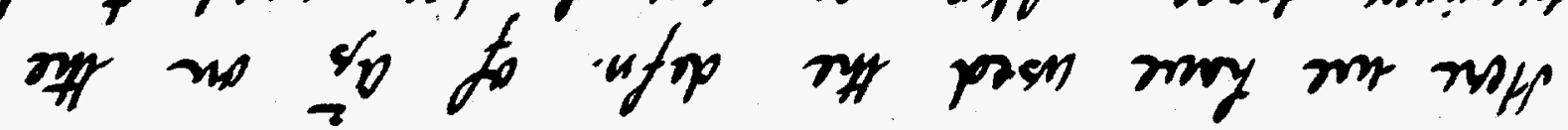

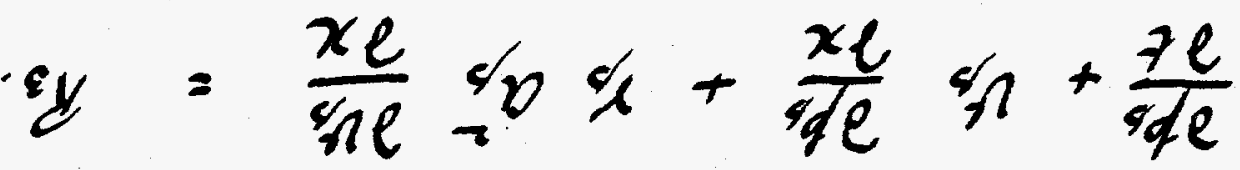

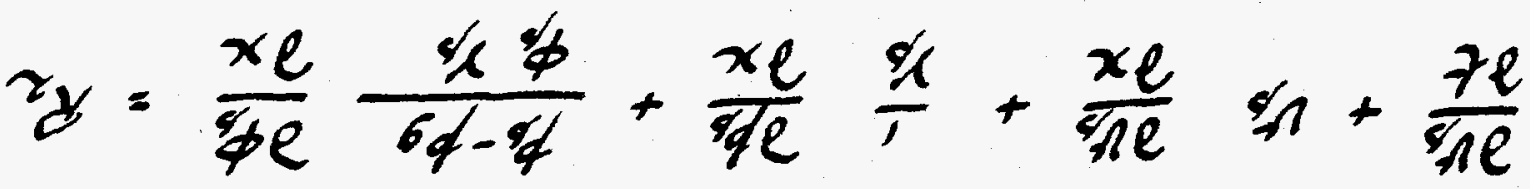

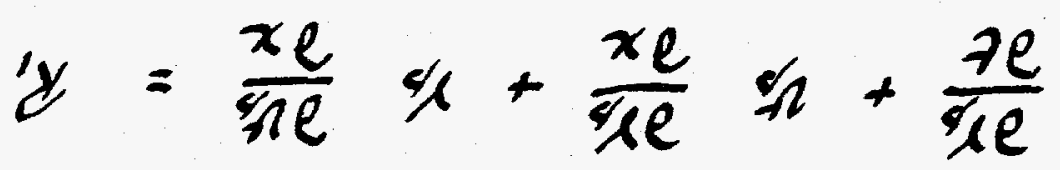

por of smm

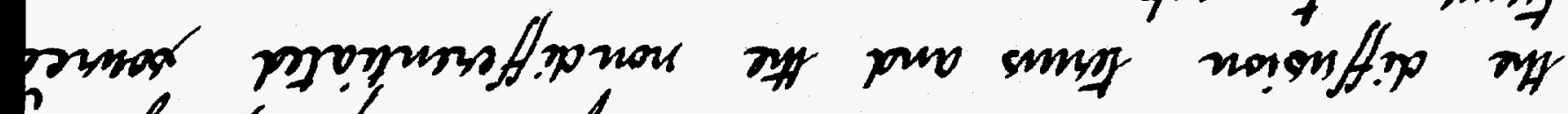

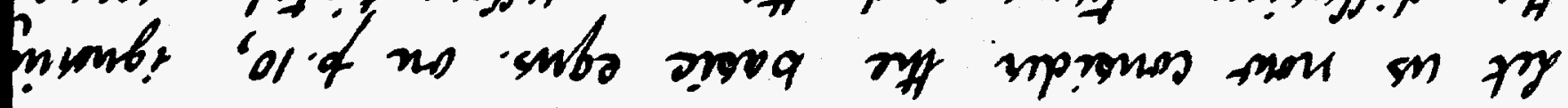

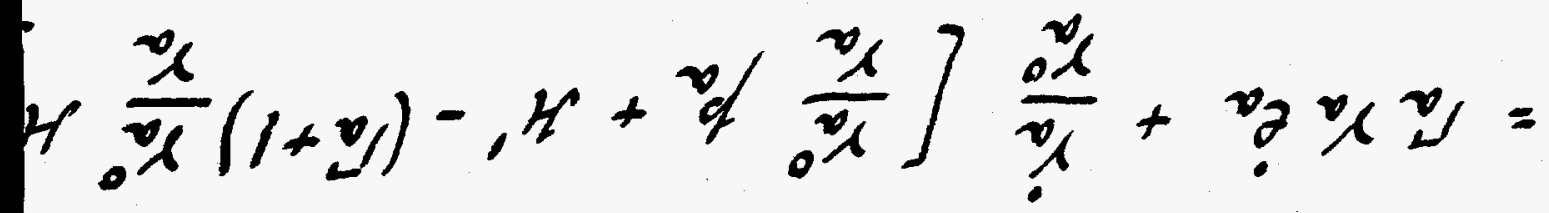

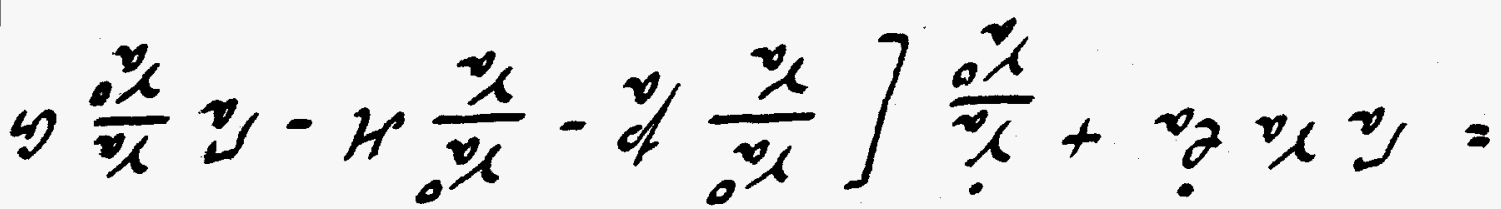

$$
\begin{aligned}
& 4 \frac{0 \%}{y}+
\end{aligned}
$$

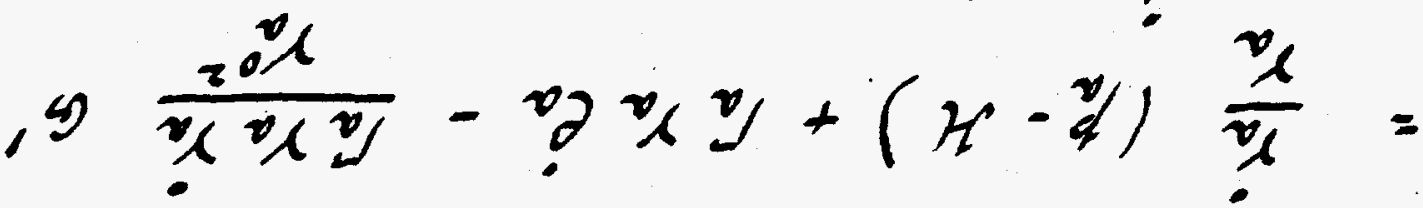

$$
\begin{aligned}
& \because, H \frac{0^{x}}{1}+
\end{aligned}
$$

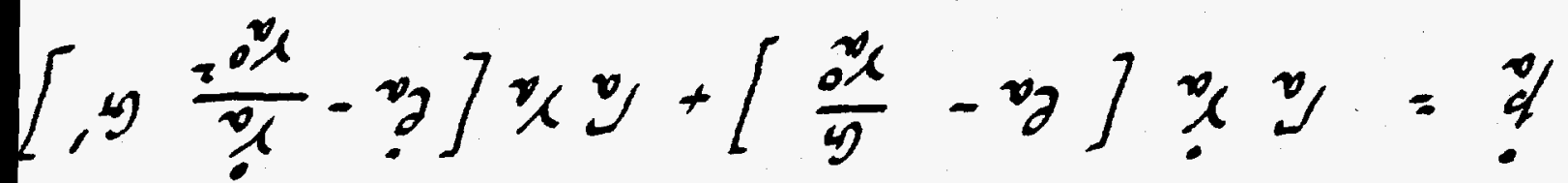


9.

equs. above correspoond precipaly to thir comberparts on 13 , provided the gencralized Lefn. of $a_{s}$ is used. Nows, the cheracteristic spech are the sane as befor. The definitions of pounce terus $R_{i}$, will, if courke, reflect the changes in the equ. of state. 


\section{ITEM 3}

sos 


\title{
Asymptotic Reduction of Two-Phase DDT Models
}

\author{
A. Kapila \\ Rensselaer Polytechnic Institute
}

Acknowledgements

M. Baer

J. Bdzil

D. Drew 


\section{The Baer-Nunziato Model}

\section{Masts}

$$
\begin{aligned}
\frac{\partial}{\partial t}\left(\phi_{s} \rho_{s}\right)+\frac{\partial}{\partial x}\left(\phi_{s} \rho_{s} v_{s}\right) & =C \\
\frac{\partial}{\partial t}\left(\phi_{g} \rho_{g}\right)+\frac{\partial}{\partial x}\left(\phi_{g} \rho_{g} v_{g}\right) & =-\sigma^{2}
\end{aligned}
$$

\section{Monientum}

$$
\begin{aligned}
& \text { b) }=
\end{aligned}
$$

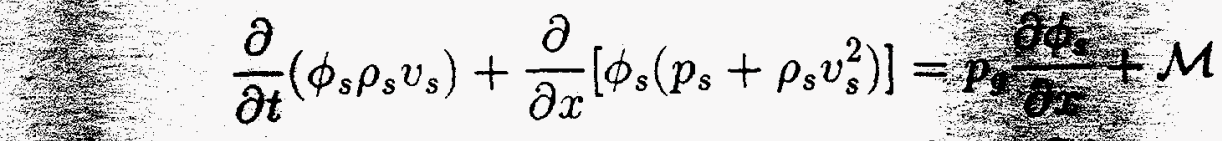

$$
\begin{aligned}
& \text { 2.t. } \\
& \frac{\partial}{\partial t}\left(\phi_{g} \rho_{g} v_{g}\right)+\frac{\partial}{\partial x}\left[\phi_{g}\left(p_{g}+\rho_{g} v_{g}^{2}\right)\right]=\frac{v_{0} \phi_{1}}{\rho_{1}} \mathcal{M}
\end{aligned}
$$

\section{Enerity}

$$
\begin{aligned}
& \text { 기난 } \\
& \frac{\frac{\partial}{\partial t}}{\partial t}\left[\phi_{s} \rho_{s}\left\{e_{s}+(1 / 2) v_{s}^{2}\right\}\right]+\frac{\partial}{\partial x}\left[\phi_{s} \rho_{s} v_{s}\left\{e_{s}+(j / \rho) \rho_{t}+(1 / 2) v_{s}^{2}\right\}\right] \\
& =\frac{\partial}{\partial x}\left(\kappa_{s} \frac{\partial T_{s}}{\partial x}\right)-\left(p_{s}-\beta_{s}\right) \mathcal{F}+p_{g} v_{\partial-\rho_{j}}+\mathcal{E}
\end{aligned}
$$

$$
\begin{aligned}
& \frac{\partial}{\sigma_{t}}\left\{\phi_{g} \rho_{g}\left\{e_{g}+(1 / 2) v_{g}^{2}\right\}\right]+\frac{\partial}{\partial x}\left[\phi_{g} \rho_{g} v_{g}\left\{e_{g}+\left(1 / f_{\rho}\right)+(1 / 2) v_{g}^{2}\right\}\right] \\
& =\frac{\partial}{\partial x}\left(\kappa_{g} \frac{\partial T_{g}}{\partial x}\right)+\left(p_{s}-\beta_{s}\right) \mathcal{F}-p_{g} v_{s} \frac{e_{x}}{\sigma_{x}}=\mathcal{E}
\end{aligned}
$$

\section{Compaction}

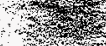

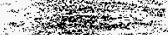

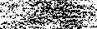

Saturtion

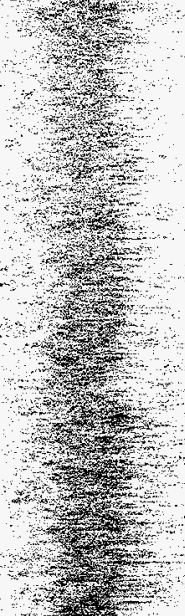$$
\frac{\partial \phi_{s}}{\partial t}+v_{s} \frac{\partial \phi_{s}}{\partial x}=\mathcal{F}+\frac{\mathcal{C}}{\rho_{s}}
$$

$$
\phi_{s}+\phi_{g}=1
$$


Interaction terms

$$
\begin{gathered}
C=\rho_{s} \mathcal{R} \\
\mathcal{M}=\mathcal{C} \frac{v_{s}+v_{g}}{2}+\delta\left(v_{g}-v_{s}\right) \\
\mathcal{E}=\left(e_{s}+\frac{1}{2} v_{s}^{2}\right) \mathcal{C}-H\left(T_{s}-T_{g}\right)-\left(\delta+\frac{1}{2} \mathcal{C}\right)\left(v_{s}-v_{g}\right) v_{s}
\end{gathered}
$$

Compaction term

$$
\mathcal{F}=\frac{\phi_{s} \phi_{g}}{\mu_{c}}\left(p_{s}-\beta_{s}-p_{g}\right)
$$

Configuration stress

$$
\beta_{s}=\beta\left(\phi_{s}\right), \beta_{s}^{\prime}>0
$$


Equations of state

Gas

$$
e_{g}=\frac{p_{g}}{\Gamma_{g} \rho_{g}}, \quad p_{g}=\Gamma_{g} \rho_{g} C_{v g} T_{g}
$$

Solid (general)

$$
\begin{gathered}
e_{s}=C_{v s} T_{s}+\frac{\Lambda}{\rho_{s 0}} g\left(\rho_{s} / \rho_{s 0}\right), p_{s}=\Gamma_{s} \rho_{s} C_{v s} T_{s}-\Lambda h\left(\rho_{s} / \rho_{s 0}\right) \\
e_{s}=\frac{p_{s}+\Lambda G\left(\rho_{s} / \rho_{s 0}\right)}{\Gamma_{s} \rho_{s}}
\end{gathered}
$$

provided

$$
h(\sigma)=-\sigma^{2} g^{\prime}(\sigma), \quad G(\sigma)=\Gamma_{s} \sigma g(\sigma)-\sigma^{2} g^{\prime}(\sigma)
$$

Solid for illustration

$$
g(\sigma)=\frac{1}{\sigma}
$$

whence

$$
e_{s}=\frac{p_{s}+\Lambda\left(\Gamma_{s}+1\right)}{\Gamma_{s} \rho_{s}}, p_{s}=\Gamma_{s} \rho_{s} C_{v s} T_{s}-\Lambda
$$

$\Lambda$ measures the degree of deviation from nonideal behavior. 


\section{Remarks}

- Model founded on mixture-theory arguments

- Seventh-order system

$$
\rho_{s}, v_{s}, p_{s}, \phi_{s}, \rho_{g}, v_{g}, p_{g}
$$

- Input needed for $\mathcal{R}, \delta, H$ and $\beta_{s}$

- nonconservative ( $\mathrm{R}-\mathrm{H}$ conditions not avallablo, 4 , trivert, steady compaction wave is a third-order system)

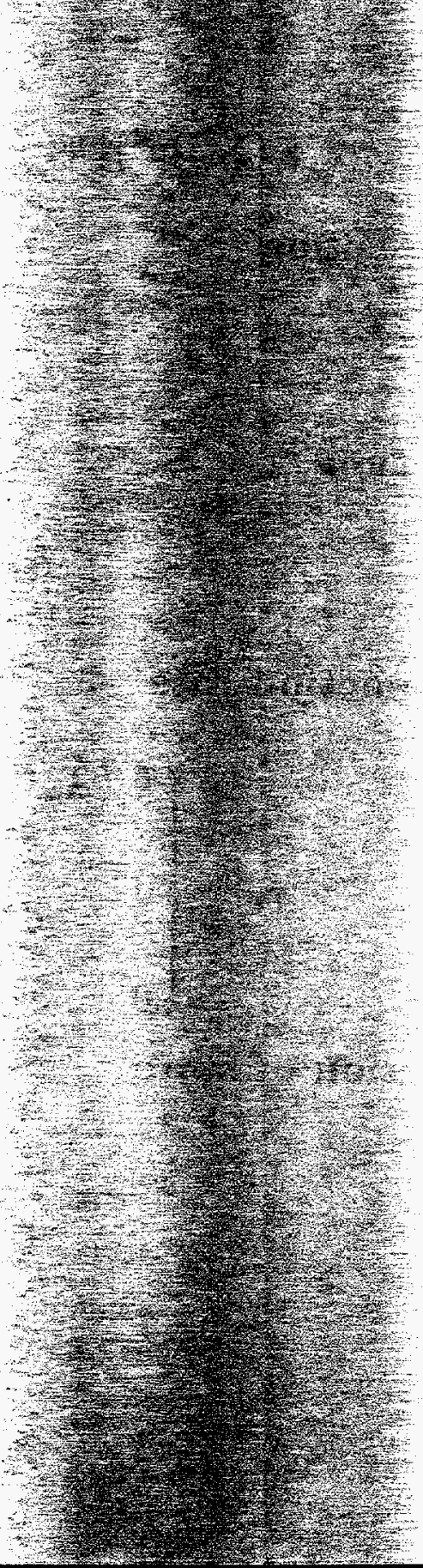




\section{The Stewart-Prasad-Asay Model}

Mass

$$
\frac{\partial \rho}{\partial t}+\frac{\partial}{\partial x}(\rho v)=0
$$

Momentum

$$
\frac{\partial}{\partial t}(\rho v)+\frac{\partial}{\partial x}\left(p+\rho v^{2}\right)=\mu \frac{\partial^{2} v}{\partial x^{2}}
$$

Energy

$$
\frac{\partial}{\partial t}\left[\rho\left\{e+(1 / 2) v^{2}\right\}\right]+\frac{\partial}{\partial x}\left[\rho v\left\{e+(1 / 2) v^{2}\right\}+p v\right]=\mu \frac{\partial^{2}}{\partial x^{2}}\left[(1 / 2) v^{2}\right]
$$

Compaction

$$
\frac{\partial \phi}{\partial t}+v \frac{\partial \phi}{\partial x}=r_{\phi}
$$

Reaction

$$
\frac{\partial \lambda}{\partial t}+v \frac{\partial \lambda}{\partial x}=r_{\lambda}(\lambda, p, \rho)
$$

Compaction rate

$$
r_{\phi}= \begin{cases}k_{\phi}(A p-\phi) & \text { for } A p-\phi>0, \text { and } \phi<1 \\ 0 & \text { for } A p-\phi<0, \text { and } \phi<1 \\ 0 & \text { for } \phi=1\end{cases}
$$

Equation of state

$$
e=\frac{1}{\gamma-1} \frac{p}{\rho}-Q_{\phi}(1-\phi)-Q_{\lambda} \lambda
$$




\section{Remarks}

- A postulated model

- Fifth-order system

$$
\rho, v, p, \phi, \lambda
$$

- Input needed for $r_{\lambda}, Q_{\lambda}, Q_{\phi}, k_{\phi}, A$

- Nonconservative (Inert, steady compaction first-order) 


\section{Questions}

- Connections between the two models?

- Any parametric limits under which B-N reduces to an effectively single-phase description? Are these limits reasonable?

- Properties of the reduced system. 

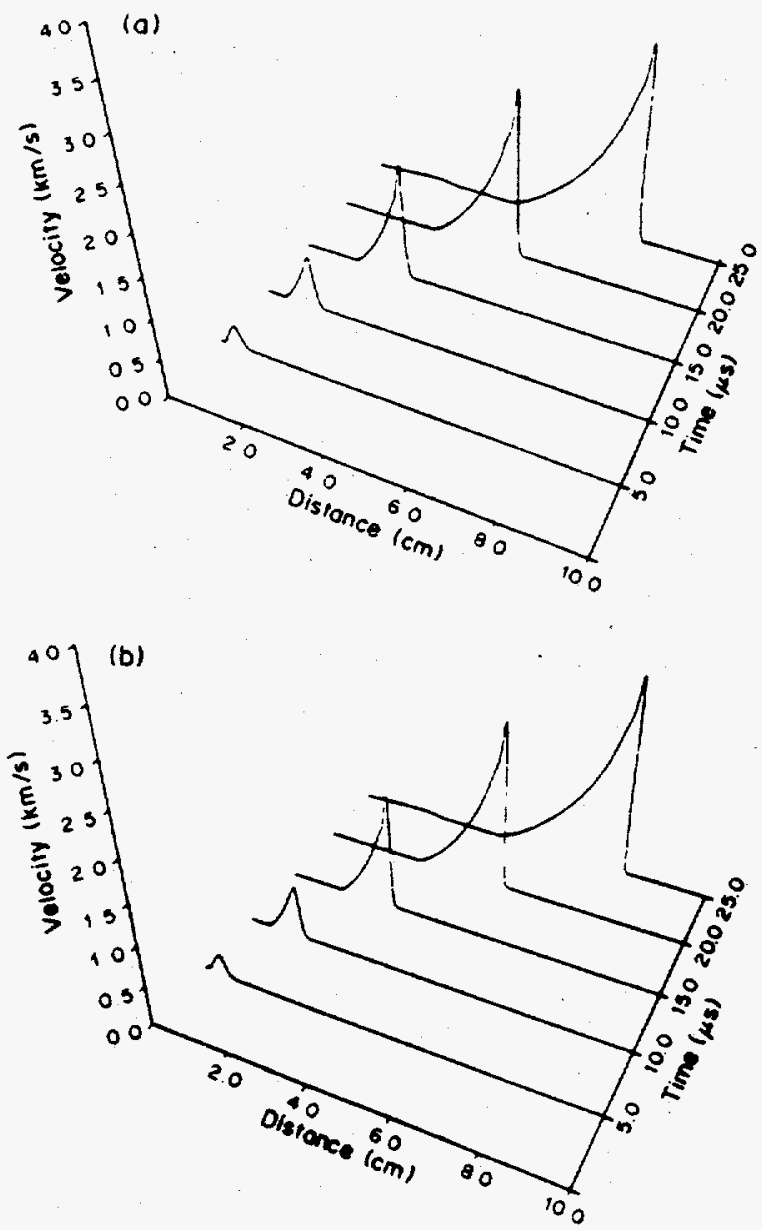

Figure 6. Temporal and spativl variations of (a) gas and (b) solid velocity durins combution of $70 \%$ dense HMX. 


\section{Asymptotic Reduction of B-N}

Numerical calculations with B-N suggest that to an excellent approximation, the two phases move together. In the model, this behavior is ensured by the limit of large drag coefficient,

$$
\delta>>1 .
$$

Then,

$$
v_{g}=v+O(1 / \delta), \quad v_{s}=v+O(1 / \delta) .
$$

Useful mixture properties

$$
\begin{gathered}
\rho=\phi_{s} \rho_{s}+\phi_{g} \rho_{g} \\
p=\phi_{s} p_{s}+\phi_{g} p_{g} \\
\rho e=\phi_{s} \rho_{s} e_{s}+\phi_{g} \rho_{g} e_{g}
\end{gathered}
$$




\section{Reformulation of B-N}

Mixture Equations

Mass

$$
\frac{\partial}{\partial t}\left(\phi_{s} \rho_{s}+\phi_{g} \rho_{g}\right)+\frac{\partial}{\partial x}\left[\left(\phi_{s} \rho_{s}+\phi_{g} \rho_{g}\right) v\right]=0
$$

Momentum

$$
\frac{\partial}{\partial t}\left[\left(\phi_{s} \rho_{s}+\phi_{g} \rho_{g}\right) v\right]+\frac{\partial}{\partial x}\left[\left\{\phi_{s} p_{s}+\phi_{g} p_{g}\right\}+\left\{\phi_{s} \rho_{s}+\phi_{g} \rho_{g}\right\} v^{2}\right]=0
$$

Energy

$$
\begin{gathered}
\frac{\partial}{\partial t}\left[\left(\phi_{s} \rho_{s} e_{s}+\phi_{g} \rho_{g} e_{g}\right)+(1 / 2)\left(\phi_{s} \rho_{s}+\phi_{g} \rho_{g}\right) v^{2}\right]+\frac{\partial}{\partial x}\left[\left\{\left(\phi_{s} \rho_{s} e_{s}+\phi_{g} \rho_{g} e_{g}\right)\right.\right. \\
\left.\left.+(1 / 2)\left(\phi_{s} \rho_{s}+\phi_{g} \rho_{g}\right) v^{2}+\left(\phi_{s} p_{s}+\phi_{g} p_{g}\right)\right\} v\right]=\frac{\partial}{\partial x}\left(\kappa_{s} \frac{\partial T_{s}}{\partial x}+\kappa_{g} \frac{\partial T_{g}}{\partial x}\right)
\end{gathered}
$$

In terms of the mixture variables,

Mass

$$
\frac{\partial \rho}{\partial t}+\frac{\partial}{\partial x}(\rho v)=0
$$

Momentum

$$
\frac{\partial}{\partial t}(\rho v)+\frac{\partial}{\partial x}\left(p+\rho v^{2}\right)=0
$$

Energy

$$
\frac{\partial}{\partial t}\left[\rho\left\{e+(1 / 2) v^{2}\right\}\right]+\frac{\partial}{\partial x}\left[\left(\rho\left\{e+(1 / 2) v^{2}\right\}+p v\right]=\frac{\partial}{\partial x}\left(\kappa_{s} \frac{\partial T_{s}}{\partial x}+\kappa_{g} \frac{\partial T_{g}}{\partial x}\right)\right.
$$




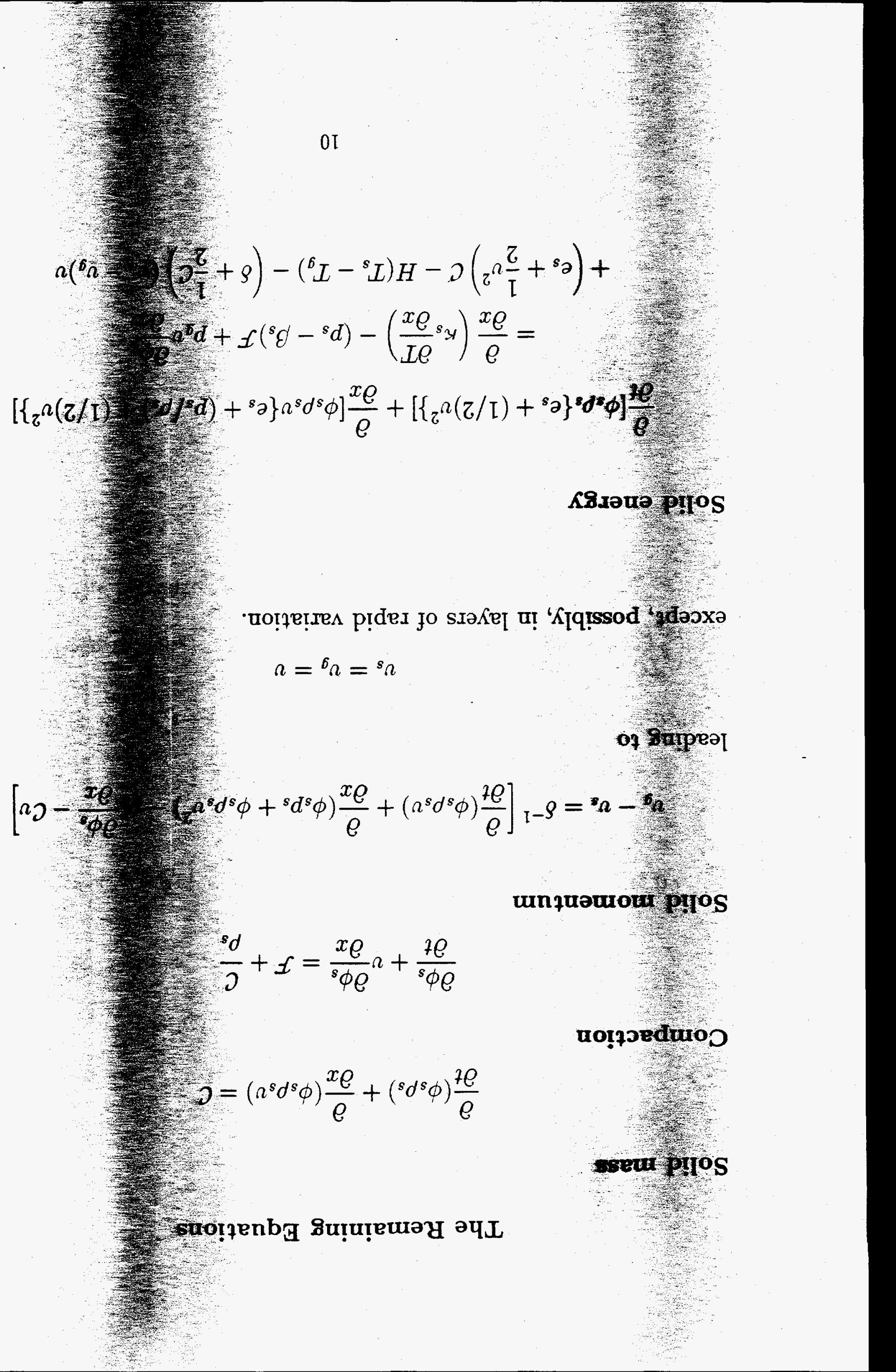


Substantial simplification under the additional limit

$$
H>>1,
$$

leading to

$$
T_{s}=T_{g}=T
$$

except, possibly, in regions of rapid variation. 


\section{Summary of reduced model}

Mass

$$
\frac{\partial \rho}{\partial t}+\frac{\partial}{\partial x}(\rho v)=0
$$

Momentum

$$
\frac{\partial}{\partial t}(\rho v)+\frac{\partial}{\partial x}\left(p+\rho v^{2}\right)=0
$$

Energy

$$
\frac{\partial}{\partial t}\left[\rho\left\{e+(1 / 2) v^{2}\right\}\right]+\frac{\partial}{\partial x}\left[\left(\rho v\left\{e+(1 / 2) v^{2}\right\}+p v\right]=\frac{\partial}{\partial x}\left(\kappa \frac{\partial T}{\partial x}\right)\right.
$$

Solid mass

$$
\frac{\partial}{\partial t}\left(\phi_{s} \rho_{s}\right)+\frac{\partial}{\partial x}\left(\phi_{s} \rho_{s} v\right)=\mathcal{C}
$$

Compaction

$$
\frac{\partial \phi_{s}}{\partial t}+v \frac{\partial \phi_{s}}{\partial x}=\mathcal{F}+\frac{\mathcal{C}}{\rho_{s}}
$$

Equality of temperatures

$$
T_{s}=T_{g}=T
$$

implies

$$
p_{s}=p_{s}\left(p, \rho, \phi_{s}, \rho_{s}\right)
$$

For the demonstration solid,

$$
\frac{p_{s}+\Lambda}{\Gamma_{s} C_{v s} \rho_{s}}=\frac{p-\phi_{s} p_{s}}{\Gamma_{g} C_{v g}\left(\rho-\phi_{s} \rho_{s}\right)}
$$

Equation of state

$$
\rho e=\frac{p}{\Gamma_{g}}+\left(\frac{1}{\Gamma_{s}}-\frac{1}{\Gamma_{g}}\right) p_{s} \phi_{s}+\frac{\Lambda\left(\Gamma_{s}+1\right)}{\Gamma_{s}} \phi_{s}
$$




\section{Remarks}

- Asymptotic limits $\delta>>1, H>>1$

- Fifth-order system

$$
p, \rho, v, \phi_{s}, \rho_{s}
$$

- Formally, SPA-like; $p_{s}$ playing the role of Xt

- Frozen sound speed is

$$
c^{2}=\left(\frac{1}{\rho \partial e / \partial p}\right)\left(\frac{p}{\rho}-\rho \frac{\partial e}{\partial \rho}\right.
$$

- Hyperbolic system, characteristic speeds

$$
v, v, v, v \pm c
$$




\section{Inert, Steady Compaction}

\section{Upstream data}

$\phi_{s 0}, T_{0}$, and $p_{s 0}$ are prescribed.

Solid equation of state determines $\rho_{s 0}$.

Compaction equilibrium determines $p_{g 0}$.

Gas equation of state determines $\rho_{g 0}$.

Definitions determine $p_{0}$ and $\rho_{0}$.

Wave speed $V$ is given. Piston speed $v$ is to be found.

Using a wave-fixed frame, $u=v-V$.

\section{Jump conditions}

Mass

$$
\rho u=-\rho_{0} V
$$

Momentum

$$
p-\rho_{0} V u=p_{0}+\rho_{0} V^{2}
$$

Solid mass

$$
\phi_{s} \rho_{s} u=-\phi_{s 0} \rho_{s 0} V
$$

Compaction equilibrium

$$
p=p_{s}-\left(1-\phi_{s}\right) \beta_{s}
$$

Energy

$$
e+\frac{1}{2} u^{2}+\frac{p}{\rho}=e_{0}+\frac{1}{2} V^{2}+\frac{p_{0}}{\rho_{0}}
$$


Equal temperatures

$$
p=\phi_{s} p_{s}+\epsilon\left(p_{s}+\Lambda\right) \phi_{s}
$$

where

$$
\epsilon=\frac{\Gamma_{g} C_{v g}\left(\rho_{0}-\phi_{s 0} \rho_{s 0}\right)}{\Gamma_{s} C_{v s} \phi_{s 0} \rho_{s 0}}
$$

Equation of state

$$
e=\alpha \frac{p}{\rho}+\Lambda(1+\alpha) \frac{\phi_{s}}{\rho}
$$

where

$$
\alpha=\frac{1}{1+\epsilon}\left(\frac{1}{\Gamma_{s}}+\frac{\epsilon}{\Gamma_{g}}\right)
$$

Energy equation

$$
\begin{gathered}
\frac{u^{2}}{V^{2}}+\frac{\alpha+1}{\alpha+(1 / 2)} \frac{u}{V}\left(1+\frac{p_{0}}{\rho_{0} V^{2}}+\frac{\Lambda \phi_{s}}{\rho_{0} V^{2}}\right) \\
+\frac{\alpha+1}{\alpha+(1 / 2)}\left(\frac{1}{2}+\frac{p_{0}}{\rho_{0} V^{2}}+\frac{\Lambda \phi_{s 0}}{\rho_{0} V^{2}}\right)=0
\end{gathered}
$$

Sound speed

$$
c^{2}=\frac{\alpha+1}{\alpha} \frac{p+\Lambda \phi_{s}}{\rho}
$$


ITEM 4 
(1)

Characteristic analysis of the equal -temp. model In nonconservative form the equations are

$$
\begin{aligned}
& \rho_{t}+v \rho_{x}+\rho v_{x}=0 \\
& v_{t}+v v_{x}+\frac{1}{\rho} p_{x}=0 \\
& e_{t}+v e_{x}+\frac{p}{\rho} v_{x}=0 \\
& \left(\phi_{s}\right)_{t}+v\left(\phi_{s}\right)_{x}=\mathcal{F}+\frac{C}{\rho_{p}} \\
& \left(\rho_{s}\right)_{t}+v\left(\rho_{s}\right)_{x}+\rho_{s} v_{x}=-\frac{\rho_{s}}{\phi_{s}} \mathcal{F}
\end{aligned}
$$

Also, $e=e\left(p, \rho, \rho, \phi_{s}\right)$

and the equal-temp. condition gives

$$
p_{s}=p_{p}\left(p_{,} \rho, \rho_{s}, \phi_{s}\right) \text {. }
$$

Now, $\quad d e=e_{p} d p+e_{\rho} d \rho+e_{p} d p_{p}+e_{\phi_{p}} d \phi_{s}$

$$
\Rightarrow \quad \frac{d e}{d t}=e_{p} \frac{d p}{d t}+e_{\rho} \frac{d p}{d t}+e_{p} \frac{d P_{t}}{d t}+e_{\phi_{s}} \frac{d \phi_{s}}{d t}
$$

If $\frac{d e}{d t} \equiv \frac{\partial e}{\partial t}+v \frac{\partial e}{\partial x}$, then.

$$
\begin{aligned}
\frac{d e}{d t} & =e_{\rho} \frac{d p}{d t}-e_{\rho} \rho v_{x}-e_{\rho}\left[\rho_{\beta} v_{x}+\frac{\rho_{\rho}}{\phi_{p}} \mathcal{F}\right] \\
& +e_{\phi_{s}}\left[\mathcal{F}+\frac{c}{\rho_{\rho}}\right]
\end{aligned}
$$

One may now replace (3) by

$$
\begin{aligned}
p_{t}+v_{x}= & \frac{1}{e_{p}}\left[-\frac{p}{\rho}+\rho e_{\rho}+\rho_{s} e_{\rho}\right] v_{x} \\
& +\frac{1}{e_{p}}\left[e_{\rho_{s}} \frac{\rho_{s}}{\phi_{s}} \mathcal{F}-e_{\phi_{s}}\left(\mathcal{F}+\frac{c}{p_{s}}\right)\right]
\end{aligned}
$$

Define $\rho C^{2} \equiv-\frac{1}{e_{p}}\left[-\frac{p}{\rho}+\rho e_{\rho}+\rho_{p} e_{p}\right]$ 
(2)

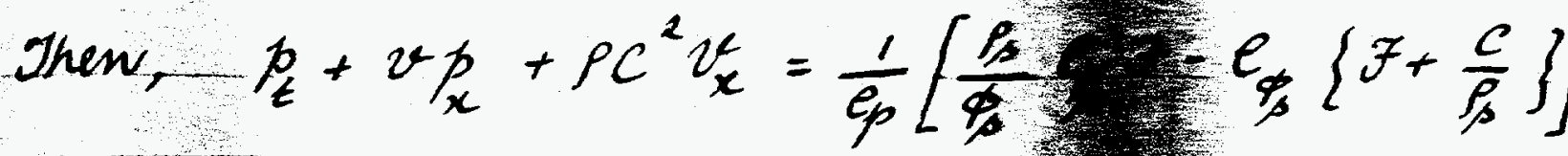

Now the equs may be written as

$$
\underline{v}_{t}+\underline{A} \underline{U}_{x}=\underline{S}
$$

where

$$
\begin{aligned}
& \underline{U}=\left\langle\rho, v, \rho_{\beta}, p, \phi_{s}\right\rangle \\
& A=\left(\begin{array}{cccc}
v & \rho & 0 & \frac{\partial}{\partial} \\
0 & v & 0 & \frac{\partial}{\partial} \\
0 & \rho_{s} & v & \frac{\sigma}{\partial} \\
0 & \rho C^{2} & 0 & \frac{v}{\sigma} \\
0 & 0 & 0 & \frac{\sigma}{z}
\end{array}\right.
\end{aligned}
$$

For eigenvalues, $\operatorname{det}(\underline{\underline{A}}-\lambda \underline{I})=$

$$
\begin{aligned}
& \Rightarrow(v-\lambda)^{3}\left[(v-\lambda)^{2}-c^{2}\right]=0 \\
& \Rightarrow \lambda=v, v, v, v \pm c .
\end{aligned}
$$


(3)

3.

characteristic Analysis of the Se ln. model $\left(v_{s}=v_{g}=v\right)$

Ignoring the pounce terms, the rations are

$$
\begin{aligned}
& \rho_{t}+v \rho_{x}+\rho v_{x}=0 \\
& v_{t}+u v_{x}+\frac{1}{\rho} p_{x}=0 \\
& e_{t}+v e_{x}+\frac{p p}{p} v_{x}=0 \\
& \left(\phi_{p}\right)_{t}+v\left(\phi_{s}\right)_{x}=0 \\
& \left(\rho_{s}\right)_{t}+v\left(P_{s}\right)_{x}+P_{s} v_{x}= \\
& \left(e_{s}\right)_{t}+v\left(e_{s}\right)_{x}+\frac{p_{s}}{p_{s}} v_{x} \\
& \text { Let }\left(\dot{)}=\frac{\partial()}{\partial t}+v \frac{\partial()}{\partial x}\right. \\
& \text { Now, }, \quad e_{\beta}=e_{\beta}\left(p_{p}, \rho_{\beta}\right) \\
& \therefore \frac{e_{s}}{\partial p_{s}} \dot{p}_{s}+\frac{\partial e_{s}}{\partial \rho_{s}} \dot{\rho}_{s} \\
& =\frac{\partial e_{s}}{\partial \rho_{s}} \dot{p}_{s}-\frac{\partial e_{s}}{\partial \rho_{\beta}} \nu_{x} \\
& \therefore \frac{\tilde{p}_{\rho}}{\partial e_{p} / \partial p_{\beta}}\left[\dot{e}_{\beta}+\frac{\partial e_{s}}{\partial \rho_{\beta}}\right. \\
& =\frac{1}{\partial e_{s} / \partial p_{s}}\left[-\frac{p_{s}}{p_{\beta}}+\frac{\partial}{\partial p}\right] v_{x}
\end{aligned}
$$

Define

$$
\rho_{s} c_{s}^{2}=\frac{1}{\partial e_{\beta} / \partial p_{s}}\left[\frac{\beta}{\rho_{\beta}}\left[\frac{\partial e_{\beta}}{\rho_{\beta}} \rho_{\beta}\right]\right.
$$


(4)

Then, solid energy eon. may be replaced by

$$
(F)_{t}+v\left(p_{b}\right)_{x}+\rho_{\beta} c_{\beta}^{2} v_{x}=0 \text {. }
$$

Similarly,

$$
\begin{aligned}
& e=e\left(p, \rho, p_{p}, \rho_{s}, \phi_{s}\right) \\
& \dot{e}=-\frac{\partial e}{\partial p} \dot{p}+\frac{\partial e}{\partial \rho} \dot{\rho}+\frac{\partial e}{\partial p_{p}} \dot{p}_{s}+\frac{\partial e}{\partial \rho_{s}} \dot{\rho}_{s}
\end{aligned}
$$

(In the absence of source terms, $\dot{\phi}_{s}=0$ )

$$
\begin{aligned}
& =\frac{\partial e}{\partial p} \dot{\rho}+V_{x}\left[-\frac{\partial e}{\partial \rho} \rho-\frac{\partial e}{\partial \rho_{\beta}} \rho_{\beta}^{2}-\frac{\partial e}{\partial \rho_{\beta}} \rho_{\beta}\right. \\
& \dot{p}=\frac{-1}{e_{\beta}}\left[+\frac{p}{\rho}-\rho \frac{\partial e}{\partial \rho}-\rho_{\beta} c_{\beta}^{2} \frac{\partial e}{\partial p_{\beta}}-\rho_{\beta} \frac{\partial e}{\partial \rho_{\beta}}\right]
\end{aligned}
$$

Define

$$
\rho c^{2}=\frac{1}{e_{\rho}}\left[\frac{p}{\rho}-\rho \frac{\partial e}{\partial \rho}-\rho_{\beta} \frac{\partial e}{\partial \rho_{\beta}}-\rho_{\beta} c_{\rho}^{2} \frac{\partial e}{\partial p_{\beta}}\right.
$$

Then the bulk energy en. may be replaced by

$$
p_{t}+v p_{x}+\rho c^{2} v_{x}=0
$$


(5)

5.

Now define $\underline{U}=\left\langle\rho, v, p_{,}, \phi_{p}, \rho_{s}, p_{p}\right\rangle$. Thew the equs. become

$\underline{u}_{t}+\stackrel{A}{=} \underline{U}_{x}=0$

whore now,

$$
A=\left(\begin{array}{llllll}
v & \rho & 0 & 0 & 0 & 0 \\
0 & v & \frac{1}{\rho} & 0 & 0 & 0 \\
0 & \rho c^{2} & v & 0 & 0 & 0 \\
0 & 0 & 0 & v & 0 & 0 \\
0 & \rho & 0 & 0 & v & 0 \\
0 & \rho_{s} c_{s}^{2} & 0 & 0 & 0 & v
\end{array}\right)
$$

Let $A-\lambda \stackrel{I}{\equiv}=0 \quad \Rightarrow$

$$
\begin{aligned}
& (v-\lambda)^{4}\left[(v-\lambda)^{2}-c^{2}\right]=0 \\
& \Rightarrow \quad \lambda=v, v, v, v, v \pm c .
\end{aligned}
$$

Note that the definition (4.3) for C, valid for the 6-eqn. model, reduces to the clefs. (1.8) for the seen. model because for the latter case, $e$ does not depend explicitly on $p$ 

(b)

$$
\frac{5}{\operatorname{sg}} \frac{10 y}{1}=\frac{s d e}{s e} \cdots
$$

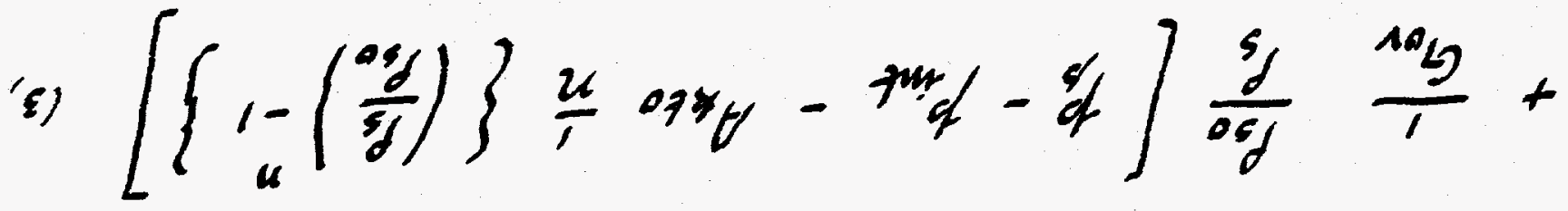

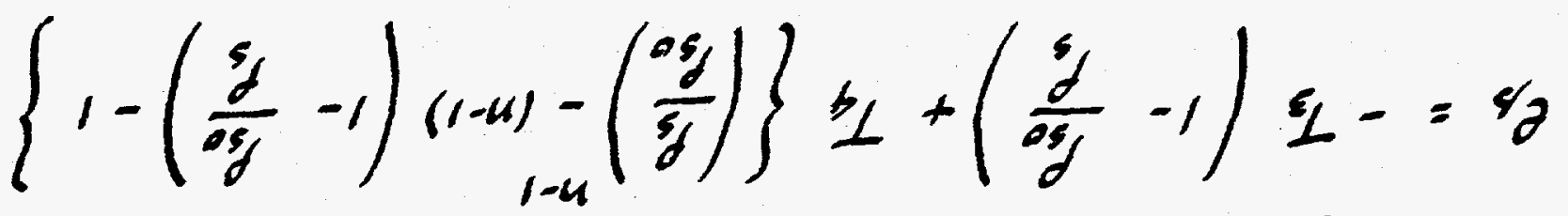

is nof himps

(v)

$$
\begin{aligned}
& \left\{1-\left(\frac{a y}{y}\right)\right\} \frac{u}{1} \text { oxy } t+ \\
& {\left[\left\{1-\left(\frac{y}{\operatorname{cog}}-1\right)(1-u)-\left(\frac{d y}{s}\right)\right\} y_{1-u}-\right.}
\end{aligned}
$$

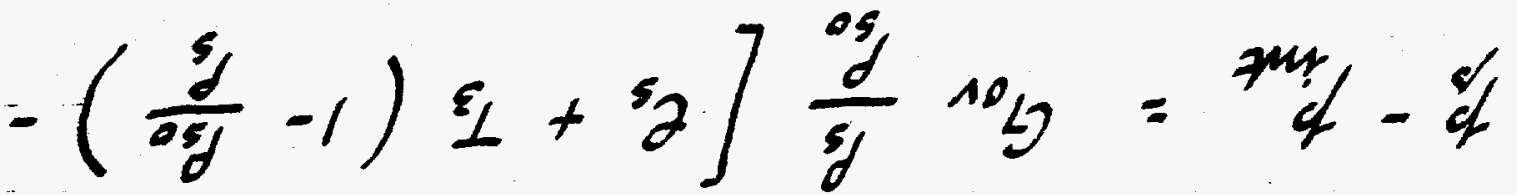

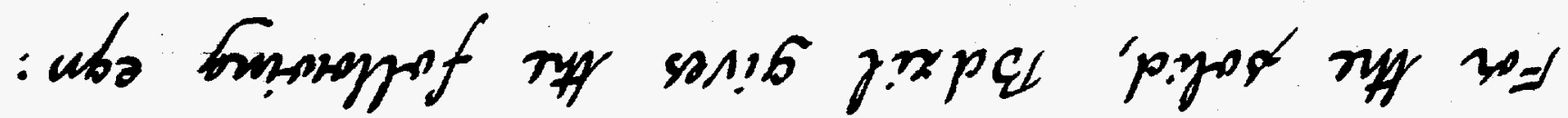

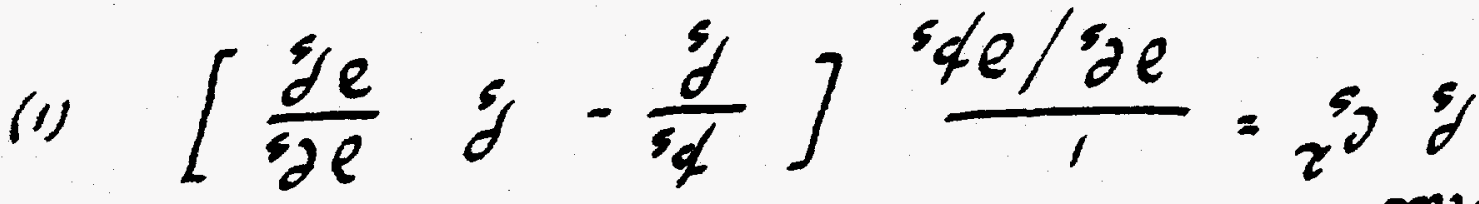

-poy noony

sy 'poods punos porot me I sposds punos fo nopromotuos 
(7)

$$
\begin{aligned}
\frac{\partial e_{s}}{\partial \rho_{s}} & =\frac{1}{\rho_{s 0}}\left(\frac{\rho_{s 0}}{\rho_{s}}\right)^{2}\left[-T_{3}+(n-1) T_{4}\left\{\left(\frac{\rho_{s}}{\rho_{s 0}}\right)^{n}-1\right\} .\right. \\
& -\frac{1}{G_{o v}}\left\{p_{s}-p_{\text {int }}-A_{k t 0} \frac{1}{n}\left[\left(\frac{\rho_{s}}{\rho_{s 0}}\right)^{n}-1\right]\right\} \\
& \left.-\frac{A_{k t 0}}{G_{\text {av }}}\left(\frac{\rho_{s}}{\rho_{s 0}}\right)^{n}\right]
\end{aligned}
$$

Thus, we can compute $\rho_{s} C_{s}^{2}$ by substituting $f$ $p_{s}$ frame (1.2), $\partial e_{s} / \partial p_{s}$ from $(1.4)$, and $\partial e_{s} / \partial P_{s}$ (2.1), into the definition (1.1).

II The mixture sound speed, $C$

Recall the definition

$$
\rho c^{2}=\frac{1}{\partial e / \partial p}\left[\frac{p}{\rho}-\rho \frac{\partial e}{\partial \rho}-\rho_{p} \frac{\partial e}{\partial \rho_{p}}-\rho_{p} c_{p}^{2} \frac{\partial e}{\partial p}\right]
$$

Here, it is assured that

$$
e=e\left(p, \rho_{1} p_{s}, \rho_{s}, \phi_{s}\right)
$$

and

$$
e_{\beta}=e_{\beta}\left(p_{\beta}, \rho_{\beta}\right) .
$$


(L)

$\frac{\phi-1}{\phi \phi-\phi}=\xi \quad, \frac{\xi-1}{\xi \phi-\delta}=\sigma$

'mapo (E) pans (v) manf rogt

(9)

$$
\frac{\sigma}{\cos \cos }=\sigma_{1}
$$

mp

(s)

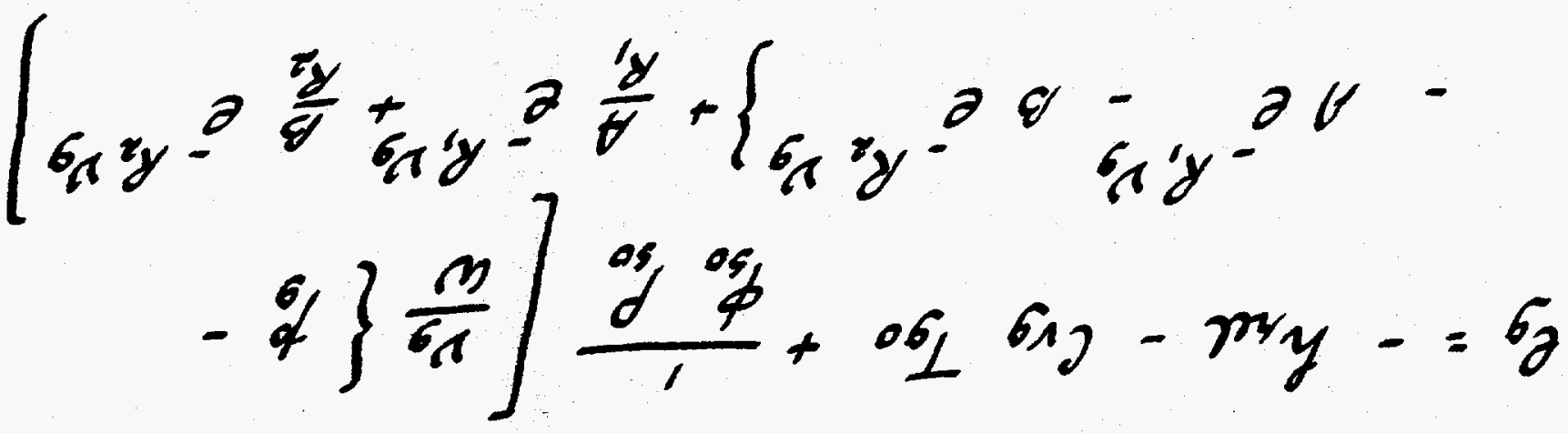

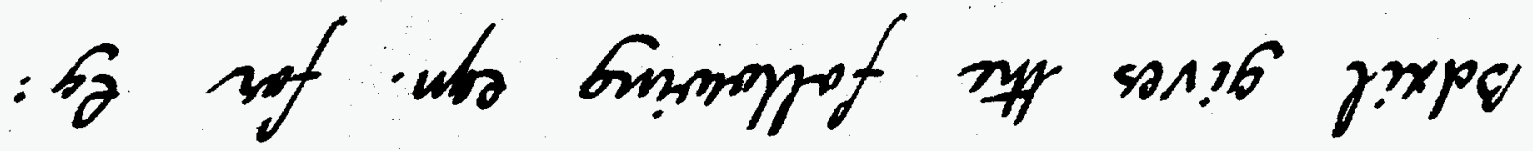

(t)

$$
\left(\sigma^{\prime} \sigma_{\phi}\right) \sigma_{z}=\sigma_{z}
$$

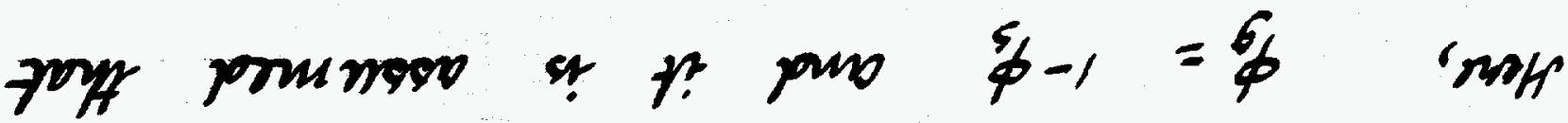

(ह)

$\sigma_{\phi} \sigma_{f}+\phi \&=q$

(2)

$\sigma_{\phi} \sigma+\phi y=\delta$

(i)

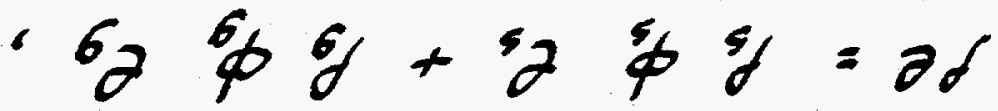

so pou. 
(9)

The last equations give

$$
\begin{aligned}
& \frac{\partial \rho_{g}}{\partial \rho}=\frac{1}{1-\phi_{s}}, \frac{\partial \rho_{g}}{\partial \rho_{s}}=\frac{-\phi_{s}}{1-\phi_{s}}, \\
& \frac{\partial p_{g}}{\partial p}=\frac{1}{1-\phi_{s}}, \frac{\partial p_{g}}{\partial p_{s}}=\frac{-\phi_{s}}{1-\phi_{s}} .
\end{aligned}
$$
Now, the formula (2.2) for $c$ opines the

$$
\frac{\partial e}{\partial p}, \frac{\partial e}{\partial \rho}, \frac{\partial e}{\partial \rho_{s}}, \frac{\partial e}{\partial p_{s}},
$$

where, from (3.1),

$$
e=\frac{\rho_{s} \phi_{s}}{\rho} e_{s}\left(p_{s}, \rho_{s}\right)+\frac{1}{\rho}\left(\rho-\rho_{s}\right) e_{g}\left(p_{g}, \rho_{g}\right)
$$

Now,

$$
\frac{\partial e}{\partial p}=\frac{1}{\rho}\left(\rho-\rho_{s} \phi_{s}\right) \frac{\partial e_{g}}{\partial p_{g}} \frac{\partial p_{g}}{\partial p}
$$

On using the deft of $\partial p_{g} / \partial p$ frame (2) above,

$$
\frac{\partial e}{\partial p}=\frac{\rho-\rho_{s} \phi_{s}}{\rho} \frac{\partial e_{g}}{\partial p_{g}} \frac{1}{1-\phi_{s}} \text {. }
$$

Similuly,

$$
\frac{\partial e}{\partial \rho}=-\frac{\rho_{s} \phi_{s}}{\rho^{2}} e_{s}+\frac{\rho_{s} \phi_{s}}{\rho^{2}} e_{g}+\left(\epsilon_{\frac{\rho}{\rho}} \frac{\rho_{s} \phi_{s}}{\rho}\right) \frac{\partial e_{g}}{\partial \rho} \frac{1}{1-g}
$$


5.

$$
\begin{aligned}
\frac{\partial e}{\partial \rho}= & \frac{\phi_{s}}{\rho} e_{s}+\frac{\rho_{s} \phi_{s}}{\rho} \frac{\partial e_{s}}{\partial \rho}-\frac{\phi_{s}}{\rho} e_{g} \\
& +\left(1-\frac{\rho_{s} \phi_{s}}{\rho}\right) \frac{\partial e_{g}}{\partial \rho_{g}}\left(\frac{-\phi_{s}}{1-\phi_{s}}\right) \\
\frac{\partial e}{\partial p_{s}}= & \frac{\rho}{\rho} \frac{\rho_{s}}{\rho} \frac{\partial e_{s}}{\partial p_{s}}+\left(1-\frac{\rho_{s} \phi_{s}}{\rho}\right) \frac{\partial e_{g}}{\partial p_{g}}\left(\frac{-\phi_{s}}{1-\phi_{s}}\right)
\end{aligned}
$$

The derivatives $\partial e_{s} / \partial p_{s}$ and $\partial e_{s} / \partial f_{s}$ have already been compacted in (1.4) and (2.1). It remains to compute $\partial e_{g} / \partial \rho_{g}$ and $\partial g / \partial \rho$ from (3.5). We get

$$
\begin{aligned}
\frac{\partial e_{g}}{\partial P_{g}} & =\frac{1}{\phi_{s o} \rho_{s o}} \frac{\nu_{g}}{\omega}=\frac{1}{\omega P_{g}} \\
\frac{\partial e_{g}}{\partial \rho_{g}} & =-\frac{1}{\rho_{g}^{2}}\left[\frac{1}{\omega}\left\{f_{g}-A e^{-R_{1} \nu_{g}}-\beta e^{-R_{2} \nu_{g}}\right\}+\right. \\
& +\frac{\nu_{g}}{\omega}\left(A R_{1} e^{-R_{1} \nu_{g}}+B R_{2} e^{-R_{2} \nu_{g}}\right) \\
& \left.-A e^{-R_{1} \nu_{g}}-B e^{-R_{2} \nu_{g}}\right]
\end{aligned}
$$

We now have all the info. needed to compute $c^{2}$. 


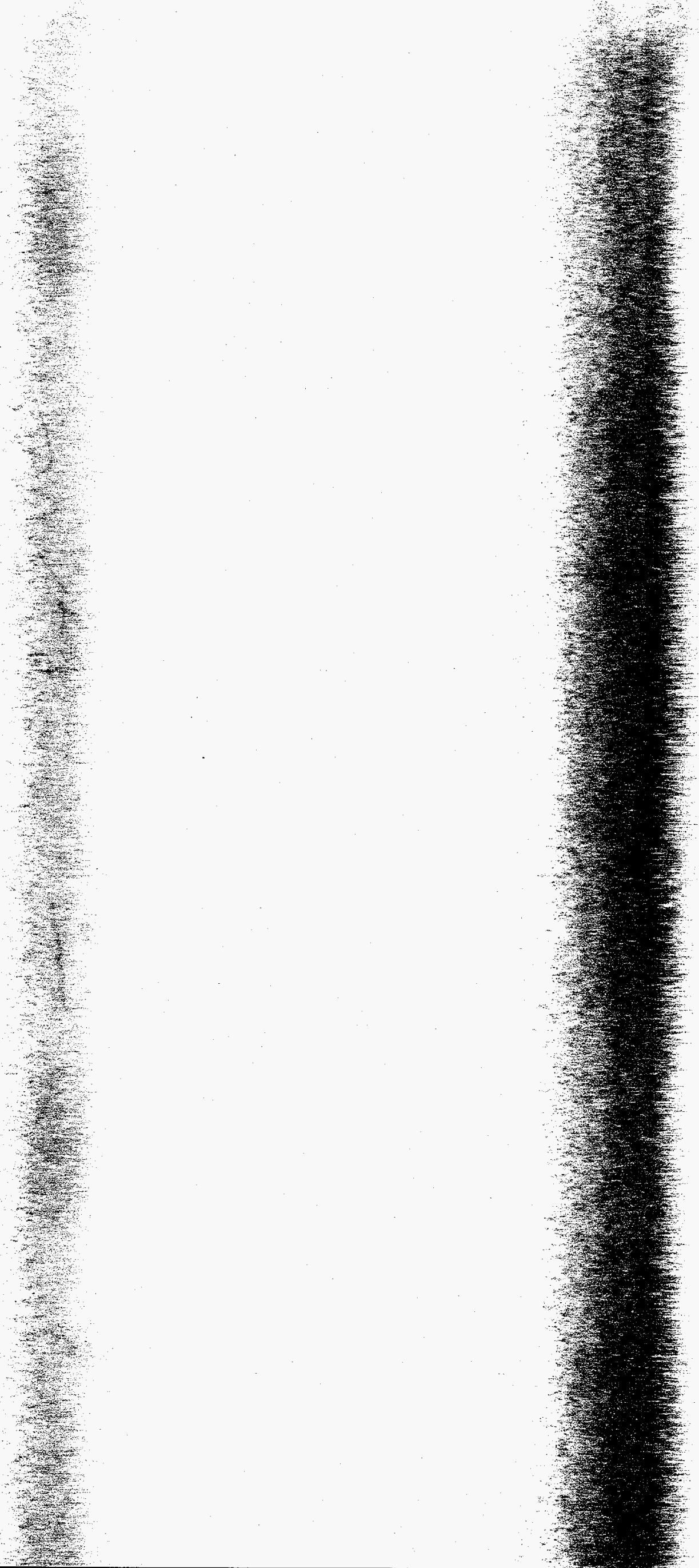


Characteristic analysis of the 6-egn. model in the solid gas form rall er. then the solectmixture form

The equations are:

mex

$$
\begin{aligned}
& \left.\frac{\partial}{\partial t}\left(\phi_{s} \rho_{s}\right)+\frac{\partial}{\partial x}\left(\phi_{p}, v\right)\right)^{c} \\
& \frac{\partial}{\partial t}\left(\phi_{g} \rho_{g}\right)+\frac{\partial}{\partial x}\left(\phi_{g}, p\right)-c
\end{aligned}
$$

momentum

$$
\begin{aligned}
& \frac{\partial}{\partial t}\left[\left(\phi_{s} \rho_{s}+\phi g\right) v\right]+\frac{\partial}{\partial x}\left[(q)\left(q g g_{g}\right) v^{2}\right. \\
& \left.+\left(\phi_{p} p_{g}+\phi_{g} p_{g}\right)\right]=0
\end{aligned}
$$

energy

$$
\begin{aligned}
& \frac{\partial}{\partial t}\left(\phi_{s} \rho_{s} e_{s}\right)+\frac{\partial}{\partial x}\left(\phi_{b} l_{s} e_{s} t\right) p_{s} \phi_{s} p_{s} \frac{\partial u}{\partial x} \\
& =C e_{s}-H\left(T_{s}-T_{g}\right)\left(j\left(p_{s}-\beta\right) \mathcal{F}\right. \\
& \frac{\partial}{\partial t}\left(g \rho_{g} e_{g}\right)+\frac{\partial}{\partial x}\left(\phi g, e_{g}\right)+\phi g \frac{\partial v}{\partial x} \\
& =-C e_{s}+H\left(T_{s}-T_{g}\right)\left(f-p_{s}\right) \mathcal{F} \\
& \frac{\partial \phi_{s}}{\partial t}+v \frac{\partial \phi_{s}}{\partial x}=\xi \frac{c}{b} .
\end{aligned}
$$


(12)

An altonate forme is

mass

$$
\begin{aligned}
& \frac{\partial}{\partial t}\left(P_{s}\right)+v \frac{\partial}{\partial x}\left(P_{s}\right)+f_{s} \frac{\partial v}{\partial x}=-\frac{P_{s}}{\phi_{s}} \mathcal{F} \\
& \frac{\partial}{\partial t}\left(P_{g}\right)+v \frac{\partial}{\partial x}\left(P_{g}\right)+P_{g} \frac{\partial u}{\partial x}=\frac{P_{g}^{F}}{\phi_{g}}-\left(1-\frac{1}{f_{3}}\right.
\end{aligned}
$$

momeactum

$$
\frac{\partial v}{\partial t}+v \frac{\partial u}{\partial x}+\frac{1}{\rho} \frac{\partial p}{\partial x}=0
$$

whene

$\rho=p_{s}+\phi_{g} \rho_{g} ; p \equiv \phi_{s} p_{0}+\phi_{g}$.

enugy

$$
\begin{aligned}
\frac{\partial e_{g}}{\partial t}+v \frac{\partial e_{s}}{\partial x}= & -\frac{1}{\phi_{s} f_{s}}\left[H\left(T_{s}-T_{g}\right)+\left(t_{s}-\beta_{s}\right) s\right. \\
& -\left(p_{s} / \rho_{s}\right) v_{x} \\
\frac{\partial e_{g}}{\partial t}+v \frac{\partial e_{g}}{\partial x}= & \frac{1}{\phi_{g} \rho_{g}}\left[\left(e_{g}-e_{s}\right) C+H\left(T_{s}-T_{g}\right)\right. \\
& -\left(p_{g} / \rho_{g}\right) v_{x}
\end{aligned}
$$

compaction

$$
\frac{\partial \phi_{s}}{\partial t}+v \frac{\partial \phi_{s}}{\partial x}=\mathcal{F}+\frac{c}{p_{s}} .
$$

Atow, egue of state:

$$
e_{g}=g_{g}\left(p_{g}, f_{g}\right), \quad e_{c}=e_{s}\left(p_{s}, f_{s}\right)
$$


3.

with a dat denoting consectuie derivativic, c.e, with

$$
\begin{aligned}
& \left(j \equiv \frac{\partial}{\partial t}()+v \frac{\partial}{\partial x}(),\right. \\
& \dot{e}_{s}=\frac{\partial e_{s}}{\partial / s} \dot{p}_{s}+\frac{\partial e_{s}}{\partial / s} \dot{l}_{s} \\
& \Rightarrow \quad \dot{p}_{s}=\frac{1}{\partial e_{s} / \partial p_{s}}\left[\dot{e}_{s}-\frac{\partial e_{s}}{\partial / s} \dot{l}_{s}\right] \\
& =\frac{1}{\partial e_{s} / \partial p_{s}}\left[-\frac{p_{s}}{\rho_{s}} \frac{\partial u}{\partial x}+\rho_{s} \frac{\partial P_{s}}{\partial \rho_{s}}\right]+\text { porerce termos }
\end{aligned}
$$

a, with

$$
\begin{gathered}
\rho_{s} c_{s}^{2} \equiv \frac{1}{\partial e_{s} / \partial p_{s}}\left[\frac{p_{s}}{p_{s}}-p_{s} \frac{\partial e_{s}}{\partial p_{s}}\right], \\
\frac{\partial p_{s}}{\partial t}+v \frac{\partial p_{s}}{\partial x}+\rho_{s} c_{s}^{2} \frac{\partial u}{\partial x}=\text { sonice terms. }
\end{gathered}
$$

similuly, for the gas,

$\frac{\partial p_{g}}{\partial t}+v \frac{\partial p_{g}}{\partial x}+p_{g} C_{g}^{2} \frac{\partial v}{\partial x}=$ sance termos, wille $G$ defined by an expression analegous to $c_{s}$.

Dhaffing the sonce thems altogetter, the systeme takes the frim

$$
\frac{\partial}{\partial t} \underline{U}+\underline{A} \frac{\partial}{\partial x} \underline{U}=0
$$


(4)

where

$(\underline{U})^{\top}=\left(\rho_{s}, \rho_{g}, v, p_{s}, p_{g}, \phi_{s}\right)$

and

$$
A=\left[\begin{array}{cccccc}
v & 0 & \rho_{s} & 0 & 0 & 0 \\
0 & v & \rho_{g} & 0 & 0 & 0 \\
0 & 0 & v & \frac{\phi}{\rho} & \frac{\phi_{g}}{\rho} & \frac{f}{\rho}\left(p_{s}-p_{g}\right) \\
0 & 0 & \rho_{s} c_{s}^{2} & v & 0 & 0 \\
0 & 0 & b g_{g}^{2} & 0 & v & 0 \\
0 & 0 & 0 & 0 & 0 & v
\end{array}\right]
$$

The eigenvalues are given by

$$
\begin{aligned}
& (v-\lambda)^{4}\left[(u-\lambda)^{2}-\frac{\phi_{s} f_{s} C_{s}^{2}+\phi_{g} P_{0} S^{2}}{\rho}\right]=0 \\
& \Rightarrow \lambda=v, v, v, u, v \pm c
\end{aligned}
$$

where $\quad \rho c^{2}=\phi_{s} P_{s} c_{s}^{2}+\phi_{g} P_{g} G^{2}$. 
sound

In the mixture formulation, the mixture, speed is given by

$$
\rho \mathcal{C}^{2}=\frac{1}{\partial e / \partial p}\left[\frac{p}{\rho}-\rho \frac{\partial e}{\partial \rho}-\rho_{s} \frac{\partial e}{\partial \rho_{s}}-\rho_{s} C_{s}^{2} \frac{\partial e}{\partial p_{s}}\right]
$$

Hers,

$$
\rho_{s} c_{s}^{2}=\frac{1}{\partial e_{s} / \partial p_{s}}\left[\frac{p_{s}}{\rho_{s}}-\rho_{s} \frac{\partial e_{s}}{\partial \rho_{s}}\right]
$$

Also,

$$
\begin{aligned}
& e=e\left(p_{,}, \rho_{s}, \rho_{s}, \phi_{s}\right) \\
& e_{s}=e_{s}\left(p_{s}, \rho_{s}\right) .
\end{aligned}
$$

Let us attempt to change the above relation for $C$ into one involving a gas/salid rather then a mixture portion formulation. Recall that

$$
\begin{aligned}
\rho e & =p_{s} \phi_{s} e_{s}+p_{g} \phi_{g} e_{g} \\
\rho & =p_{s} \phi_{g}+p_{g} \phi_{g} \\
p & =p_{s} \phi_{s}+p_{g} \phi_{g} \\
e_{g} & =e_{g}\left(p_{g}, p_{g}\right)
\end{aligned}
$$

We now compute the following derivatives of $e$ by using eqn. (5) alone. Note the arguments of $e$ in eau. (3).

$$
\rho \frac{\partial e}{\partial p}=\rho_{g} \phi_{g} \frac{\partial e_{g}}{\partial p_{g}} \frac{\partial p_{g}}{\partial p}=\rho_{g} \phi_{g} \frac{\partial e_{g}}{\partial p_{g}} \frac{1}{g}
$$

a $\frac{\partial e}{\partial p}=\frac{\rho_{g}}{\rho} \frac{\partial e_{g}}{\partial \rho}$ 
(16)

In computing $\partial e / \partial \rho_{s}$ at fixed $p_{1} \rho_{1} p_{s}$ and $\phi_{s}$, note that in equ. (1.5), $\rho_{g}$ varies. Thus,

$$
\rho \frac{\partial e}{\partial \rho_{s}}=\phi_{s} e_{s}+\rho_{s} \phi_{s} \frac{\partial e_{s}}{\partial \rho_{s}}+\phi\left[e_{g}+\rho \frac{\partial e_{g}}{\partial \rho_{g}}\right] \frac{\partial \rho_{g}}{\partial \rho_{s}}
$$

where, from $(1.6)$,

$$
\begin{gathered}
0=\phi_{s}+\phi_{g} \frac{\partial P_{g}}{\partial \rho_{s}} \Rightarrow \frac{\partial P_{g}}{\partial \rho_{s}}=-\frac{\phi_{s}}{\phi_{g}} \\
\therefore \quad \frac{\partial e}{\partial \rho_{s}}=\phi_{s} e_{s}+\rho_{s} \phi_{s} \frac{\partial e_{s}}{\partial P_{s}}-\phi_{s} e_{g}-\phi_{s} \rho_{g} \frac{\partial e_{g}}{\partial \rho_{g}} .
\end{gathered}
$$

Now we compute $\frac{\partial e}{\partial p_{s}}$ at fixed $p_{,} p_{s}, \phi_{s}$ and $p_{\text {, }}$, but with $p_{g}$ varying, such that

$$
\frac{\partial p_{g}}{\partial p_{s}}=-\frac{\phi_{s}}{\phi_{g}} \text {. }
$$

Therefore,

$$
\rho \frac{\partial e}{\partial p_{s}}=\rho_{s} \phi_{s} \frac{\partial e_{s}}{\partial p_{s}}+\rho_{g} \phi_{g} \frac{\partial e_{g}}{\partial p_{g}}\left(-\frac{\phi_{s}}{\phi_{g}}\right)
$$

$n, \rho \frac{\partial e}{\partial p_{s}}=\phi_{s} \rho_{s} \frac{\partial e_{s}}{\partial p_{s}}-\phi_{s} \rho_{g} \frac{\partial e_{g}}{\partial p_{g}}$.

Finally, we compute $\frac{\partial e}{\partial \rho}$ at fixed $p, p_{s}, p_{s}, \phi_{s}$, but with varying $P_{g}$, with $\partial P_{g} / \partial \rho=1 / \phi_{g}$. Therefore,

or,

$$
e+\rho \frac{\partial e}{\partial \rho}=\left[\phi_{g} e_{g}+\rho \phi_{g} \frac{\partial e_{g}}{\partial \rho_{g}}\right] \frac{1}{g g}
$$

$$
\rho \frac{\partial e}{\partial \rho}=-e+e_{g}+\rho_{g} \frac{\partial e_{g}}{\partial \rho_{g}}
$$


3.

We shall now substitute these derivatives in (10)) to peciturhat the simplified expression for $c$ is. Now, $(f i) \Rightarrow$

$$
\begin{aligned}
\rho c^{2} \frac{\partial e}{\partial \rho} & =\frac{p}{\rho}-\rho \frac{\partial e}{\partial \rho}-\rho_{s} \frac{\partial e}{\partial \rho_{s}}-\rho_{s} c_{s}^{2} \frac{\partial e}{\partial \beta_{s}} \\
& =\frac{p}{\rho}+\left[e \cdot e_{g}-\rho_{g} \frac{\partial e_{g}}{\partial \rho_{g}}\right] \\
& -\frac{\rho_{s}}{\rho}\left[\phi_{s} e_{s}+\rho \phi_{s} \frac{\partial e_{s}}{\partial \rho_{s}}-\phi_{s} e_{g}-\phi_{s} \rho \frac{\partial e_{g}}{\partial \rho}\right] \\
& -\rho_{s} c_{s}^{2} \frac{\partial e}{\partial \rho_{s}}
\end{aligned}
$$

Now,

$$
\begin{aligned}
& e-e_{g}-\frac{\rho_{s}}{\rho} \phi_{s}\left[e_{s}-e_{g}\right]=\frac{1}{\rho}\left[\rho e-\rho_{s} \phi_{s} e_{s}-\left[\rho-\rho_{s} \phi_{s} l e_{g}\right]\right. \\
& =\frac{\prime}{\rho}\left[\rho e-e_{c} \phi_{s} e_{s}-\rho_{g} \phi_{g} e_{g}\right] \equiv 0 \text {. } \\
& P C^{2} \frac{\partial e}{\partial p}=\frac{p}{\rho}-\rho_{g} \frac{\partial e_{g}}{\partial \rho_{g}}\left\{1-\frac{P_{s} \phi_{s}}{\rho}\right\}-\frac{\rho_{s}^{2}}{P} \phi_{s} \frac{\partial e_{s}}{\partial P_{s}} \\
& -\rho_{s} c_{s}^{2} \frac{1}{\rho}\left[\phi_{s} \rho_{s} \frac{\partial e_{s}}{\partial p_{s}}-\phi_{s} \rho_{\rho} \frac{\partial e_{g}}{\partial p_{g}}\right] \\
& =\frac{f_{f} \phi_{s}}{\rho}-\frac{\rho_{s}^{2} \phi_{s}}{\rho} \frac{\partial e_{s}}{\partial \rho_{s}}+\frac{p_{g} \phi_{g}}{\rho}-\frac{\rho_{g}^{2} \phi_{g}}{\rho} \frac{\partial e_{g}}{\partial \rho_{g}} \\
& -\frac{f \phi_{s} c_{s}^{2}}{\rho}\left[\rho_{s} \frac{\partial e_{s}}{\partial p_{s}}-\rho_{g} \frac{\partial e_{g}}{\partial p_{g}}\right] \\
& =\frac{\rho_{s} f_{s}}{\rho}\left[\frac{p_{s}}{\rho_{s}}-\rho_{s} \frac{\partial e_{s}}{\partial \rho_{s}}\right]+\frac{\rho \phi_{s}}{\rho}\left[\frac{f}{\rho_{s}}-\rho_{g} \frac{\partial e_{g}}{\partial \rho}\right]
\end{aligned}
$$


(14)

$$
-\frac{p_{s} \phi_{s} c_{s}^{2}}{\rho}\left[p_{s} \frac{\partial e_{s}}{\partial p_{s}}-\rho_{g} \frac{\partial e_{g}}{\partial p_{g}}\right]
$$

Now wee the definition

$$
\frac{\partial e_{s}}{\partial p_{s}} \rho_{s} c_{s}^{2}=\frac{p_{s}}{\rho_{s}}-\rho_{s} \frac{\partial e_{s}}{\partial \rho_{s}}
$$

and a similar one for $f_{g}$. Then,

$$
\begin{aligned}
\rho c^{2} \frac{\partial e}{\partial p}=\rho g c^{2} & \frac{\partial e_{g}}{\partial p_{g}}=\frac{\rho_{s}^{2} \phi_{s} c_{s}^{2}}{\rho} \frac{\partial e_{s}}{\partial p_{s}} \\
& +\frac{\rho_{g}^{2} \phi_{g} c_{g}^{2}}{\rho} \frac{\partial e_{g}}{\partial p_{g}} \\
& -\frac{\rho_{s} \phi_{s} c_{s}^{2}}{\rho}\left[\rho_{s} \frac{\partial e_{s}}{\partial p_{s}}-\rho \frac{\partial e_{g}}{\partial p_{g}}\right]
\end{aligned}
$$

or, $\rho_{g} c^{2} \frac{\partial e_{g}}{\partial p_{g}}=\frac{\rho_{g}}{\rho}\left[\rho_{g} \phi_{g} C_{g}^{2} \frac{\partial e_{g}}{\partial p_{g}}+\rho_{g} \phi_{s} C_{s}^{2} \frac{\partial e_{g}}{\partial p_{g}}\right]$

$N_{1} \frac{\rho}{\rho} \rho_{g} \frac{\partial e_{g}}{\partial \rho_{g}}\left[\rho C^{2}-\rho_{0} \phi_{s} c_{g}^{2}-\rho_{g} \phi_{g} C_{g}^{2}\right]=0$

$$
\Rightarrow \quad \rho c^{2}=\rho_{s} \phi_{s} c_{s}^{2}+\rho \phi_{g} c_{g}^{2}
$$

as obtained earlier. 


\section{The Six-Equation Model in Characteristic Form}

We start with the familiar, near-conservative form of the equations:

$$
\begin{array}{r}
\frac{\partial}{\partial t}\left(\phi_{s} \rho_{s}\right)+\frac{\partial}{\partial x}\left(\phi_{s} \rho_{s} v\right)=\mathcal{C}_{s}, \\
\frac{\partial}{\partial t}\left(\phi_{g} \rho_{g}\right)+\frac{\partial}{\partial x}\left(\phi_{g} \rho_{g} v\right)=-\mathcal{C}_{s}, \\
\frac{\partial}{\partial t}(\rho v)+\frac{\partial}{\partial x}\left(p+\rho v^{2}\right)=0, \\
\frac{\partial}{\partial t}\left(\phi_{s} \rho_{s} e_{s}\right)+\frac{\partial}{\partial x}\left(\phi_{s} \rho_{s} e_{s} v\right)+\phi_{s} p_{s} \frac{\partial v}{\partial x}=\mathcal{C}_{s} e_{s}-H\left(T_{s}-T_{g}\right)-\left(p_{s}-\beta_{s}\right) \mathcal{F}, \\
\frac{\partial}{\partial t}\left(\phi_{g} \rho_{g} e_{g}\right)+\frac{\partial}{\partial x}\left(\phi_{g} \rho_{g} e_{g} v\right)+\phi_{g} p_{g} \frac{\partial v}{\partial x}=-\mathcal{C}_{s} e_{s}+H\left(T_{s}-T_{g}\right)+\left(p_{s}-\beta_{s}\right) \mathcal{F}, \\
\frac{\partial}{\partial t}\left(\phi_{s}\right)+v \frac{\partial}{\partial x}\left(\phi_{s}\right)=\mathcal{F}+\frac{\mathcal{C}_{s}}{\rho_{s}} .
\end{array}
$$

The mixture quantities appearing above are defined as

$$
\rho=\phi_{s} \rho_{s}+\phi_{g} \rho_{g}, \quad p=\phi_{s} p_{s}+\phi_{g} p_{g}
$$

The compaction function is

$$
\mathcal{F}=\frac{\phi_{s} \phi_{g}}{\mu_{c}}\left(p_{s}-p_{g}-\beta_{s}\right)
$$

For characteristic calculations, it is more convenient to rewrite the equations in the following alternate form:

Mass conservation

$$
\begin{array}{r}
\frac{\partial}{\partial t}\left(\rho_{s}\right)+v \frac{\partial}{\partial x}\left(\rho_{s}\right)+\rho_{s} \frac{\partial v}{\partial x}=-\frac{\rho_{s}}{\phi_{s}} \mathcal{F} \\
\frac{\partial}{\partial t}\left(\rho_{g}\right)+v \frac{\partial}{\partial x}\left(\rho_{g}\right)+\rho_{g} \frac{\partial v}{\partial x}=\frac{\rho_{g}}{\phi_{g}} \mathcal{F}-\left(1-\frac{\rho_{g}}{\rho_{s}}\right) \frac{\mathcal{C}_{s}}{\phi_{g}}
\end{array}
$$

Momentum balance

$$
\frac{\partial v}{\partial t}+v \frac{\partial v}{\partial x}+\frac{1}{\rho}\left\{\phi_{s} \frac{\partial}{\partial x}\left(p_{s}\right)+\phi_{g} \frac{\partial}{\partial x}\left(p_{g}\right)+\left(p_{s}-p_{g}\right) \frac{\partial}{\partial x}\left(\phi_{s}\right)\right\}=0
$$

Here, the mixture pressure $p$ has been replaced by its definition (7b).

Energy balance

$$
\begin{array}{r}
\frac{\partial}{\partial t}\left(e_{s}\right)+v \frac{\partial}{\partial x}\left(e_{s}\right)+\frac{p_{s}}{\rho_{s}} \frac{\partial v}{\partial x}=-\frac{1}{\phi_{s} \rho_{s}}\left\{H\left(T_{s}-T_{g}\right)+\left(p_{s}-\beta_{s}\right) \mathcal{F}\right\} \\
\frac{\partial}{\partial t}\left(e_{g}\right)+v \frac{\partial}{\partial x}\left(e_{g}\right)+\frac{p_{g}}{\rho_{g}} \frac{\partial v}{\partial x}=\frac{1}{\phi_{g} \rho_{g}}\left\{H\left(T_{s}-T_{g}\right)+\left(p_{s}-\beta_{s}\right) \mathcal{F}+\left(e_{g}-e_{s}\right) \mathcal{C}_{s}\right\}
\end{array}
$$

The equations of state $e_{s}=e_{s}\left(p_{s}, \rho_{s}\right)$ and $e_{g}=e_{g}\left(p_{g}, \rho_{g}\right)$ can be used to recast the energy equations further. Note that

$$
\dot{e}_{s}=\frac{\partial e_{s}}{\partial p_{s}} \dot{p}_{s}+\frac{\partial e_{s}}{\partial \rho_{s}} \dot{\rho}_{s}
$$

whence

$$
\dot{p}_{s}=\frac{1}{\partial e_{s} / \partial p_{s}}\left\{\dot{e}_{s}-\frac{\partial e_{s}}{\partial \rho_{s}} \dot{\rho}_{s}\right\}
$$


The solid energy equation now yields

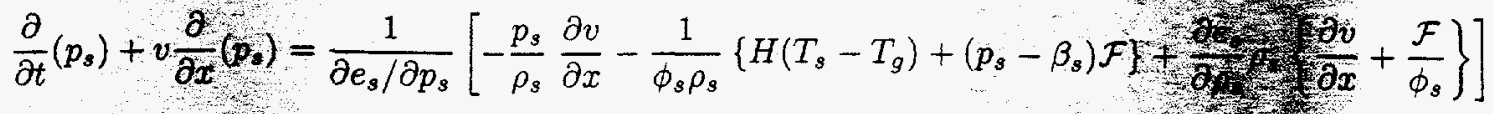

which, in view of the definition

$$
\rho_{s} c_{s}^{2}=\frac{1}{\partial e_{s} / \partial p_{s}}\left\{\frac{p_{s}}{\rho_{s}}-\rho_{s} \frac{\partial e_{s}}{\partial \rho_{s}}\right\}
$$

becomes

$$
\frac{\partial}{\partial t}\left(p_{s}\right)+v \frac{\partial}{\partial x}\left(p_{s}\right)+\rho_{s} c_{s}^{2} \frac{\partial v}{\partial x}=E_{s}
$$

where

$$
E_{s}=\frac{1}{\partial e_{s} / \partial p_{s}}\left[-\frac{1}{\phi_{s} \rho_{s}}\left\{H\left(T_{s}-T_{g}\right)+\left(p_{s}-\beta_{s}\right) \mathcal{F}\right\}+\frac{\partial e_{s}}{\partial \rho_{s}} \frac{\rho g}{\phi_{g}}\right]
$$

Equation (16) will now be treated as an alternate form of the solid energy eques? gas energy equation turns out to be

$$
\frac{\partial}{\partial t}\left(p_{g}\right)+v \frac{\partial}{\partial x}\left(p_{g}\right)+\rho_{g} c_{g}^{2} \frac{\partial v}{\partial x}=E_{g}
$$

with

$$
\left.E_{g}=\frac{1}{\partial e_{g} / \partial p_{g}}\left\{\frac{1}{\phi_{g} \rho_{g}}\left\{H\left(T_{s}-T_{g}\right)+\left(p_{s}-\beta_{s}\right) \mathcal{F}+\left(e_{g}-e_{s}\right) \mathcal{C}_{s}\right\}+\frac{\partial e_{g}}{\partial \rho_{g}}\left\{-\frac{\rho_{g}}{\phi_{g}}-\frac{\gamma}{g_{g}}\right) \frac{\mathcal{C}_{s}}{\phi_{g}}\right\}\right]
$$

and a definition similar to (15) for $c_{g}$.

To summarize, the 6 -equation model now consists of

$$
\begin{array}{r}
\frac{\partial}{\partial t}\left(\rho_{s}\right)+v \frac{\partial}{\partial x}\left(\rho_{s}\right)+\rho_{s} \frac{\partial v}{\partial x}=-\frac{\rho_{s}}{\phi_{g}} \\
\frac{\partial}{\partial t}\left(\rho_{g}\right)+v \frac{\partial}{\partial x}\left(\rho_{g}\right)+\rho_{g} \frac{\partial v}{\partial x}=\frac{\rho_{g}}{\phi_{g}} \mathcal{F}-\left(1-\frac{\rho_{g}}{\rho_{s}}\right) \\
\frac{\partial v}{\partial t}+v \frac{\partial v}{\partial x}+\frac{1}{\rho}\left\{\phi_{s} \frac{\partial}{\partial x}\left(p_{s}\right)+\phi_{g} \frac{\partial}{\partial x}\left(p_{g}\right)+\left(p_{s}-p_{g}\right) \frac{\partial}{\partial x}\left(\phi_{s}\right)\right\} \\
\frac{\partial}{\partial t}\left(p_{s}\right)+v \frac{\partial}{\partial x}\left(p_{s}\right)+\rho_{s} c_{s}^{2} \frac{\partial v}{\partial x}=\frac{1}{\partial t} \\
\frac{\partial}{\partial t}\left(p_{g}\right)+v \frac{\partial}{\partial x}\left(p_{g}\right)+\rho_{g} c_{g}^{2} \frac{\partial v}{\partial x}=v_{g} \\
\frac{\partial}{\partial t}\left(\phi_{s}\right)+v \frac{\partial}{\partial x}\left(\phi_{s}\right)=\mathcal{F}+\frac{c_{s}}{\rho_{s}}
\end{array}
$$

This hyperbolic sjstem for the vector

$$
\mathrm{U}=\left\langle\rho_{s}, \rho_{g}, v, p_{s}, p_{g}, \phi_{s}>\right.
$$

has eigenvalues $v$ (of multiplicity 4 ) and $v \pm c$. The corresponding left eigenvesto

$$
\begin{array}{r}
L_{1}=\langle 0,0,0,0,0,1> \\
L_{2}=<-\rho_{g}, \rho_{s}, 0,0,0,0> \\
L_{3}=<-c_{s}^{2}, 0,0,1,0,0> \\
L_{4}=<0,-c_{g}^{2}, 0,0,1,0>
\end{array}
$$


for the eigenvalue $v$, and

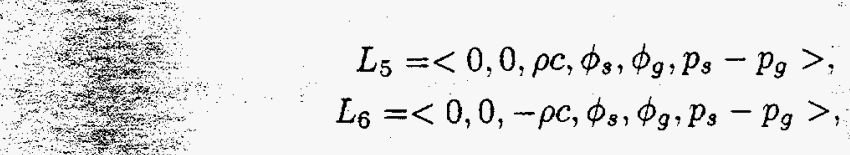

for the acoustic eigenvalues $v \pm c$. The equivalent characteristic system can now be obtained by premultiplying (20 - 25) with the left eigenvectors. After some manipulation, the followith set emerges:

$$
\begin{aligned}
& \frac{d \phi_{s}}{d t}=\mathcal{F}+\frac{\mathcal{C}_{s}}{\rho_{s}} \\
& \frac{3}{3+3} \\
& \rho_{s} \frac{d \rho_{g}}{d t}-\rho_{g} \frac{d \rho_{s}}{d t}=\frac{\rho_{s} \rho_{g}}{\phi_{s} \phi_{g}} \mathcal{F}-\frac{\rho_{s}-\rho_{g}}{\phi_{g}} \mathcal{C}_{s} \\
& \frac{d p_{s}}{d t}-c_{s}^{2} \frac{d \rho_{s}}{d t}=E_{s}+\frac{\rho_{s} c_{s}^{2}}{\phi_{s}} \mathcal{F} \\
& \frac{d p_{g}}{d t}-c_{g}^{2} \frac{d \rho_{g}}{d t}=E_{g}-\frac{\rho_{g} c_{g}^{2}}{\phi_{g}} \mathcal{F}+\left(1-\frac{\rho_{g}}{\rho_{s}}\right) \frac{c_{g}^{2}}{\phi_{g}} \mathcal{C}_{s} \\
& \text { 2.t. } \frac{d^{+} p}{d t}+\rho c \frac{d^{+} v}{d t}=\phi_{s} E_{s}+\phi_{g} E_{g}+\left(p_{s}-p_{g}\right)\left(\mathcal{\mathcal { F }}+\frac{\boldsymbol{C}_{s}}{\rho_{s}}\right) \\
& \frac{d^{-} p}{d t}-\rho c \frac{d^{-} v}{d t}=\phi_{s} E_{s}+\phi_{g} E_{g}+\left(p_{s}-p_{g}\right)\left(\mathcal{F}+\frac{c_{s}}{\rho_{s}}\right)
\end{aligned}
$$

where $d / d t$ depotes fate of change along the particle path and $d^{ \pm} / d t$ the rate $-6 /$ to along the sonic lines.
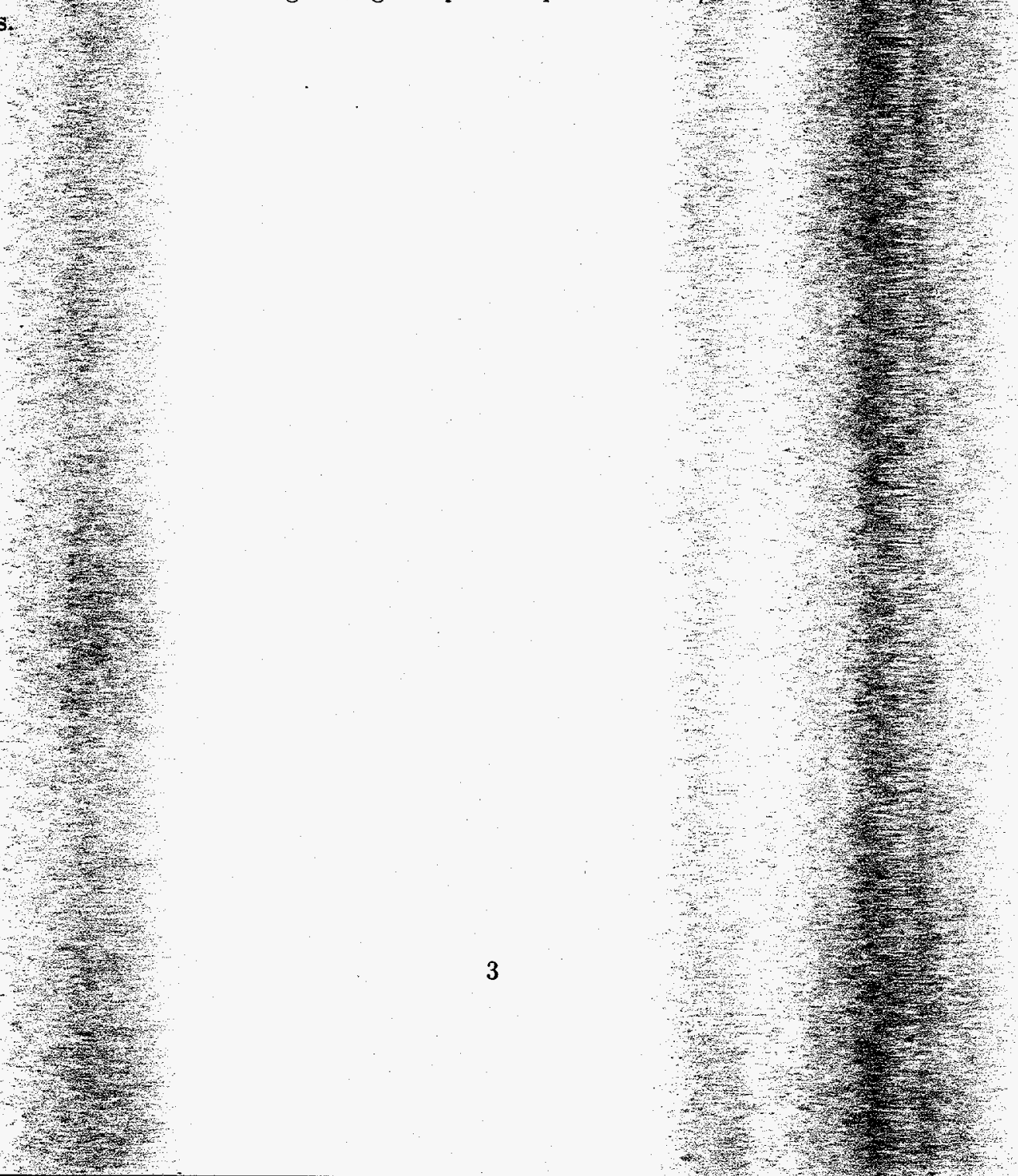

characteristics of $B K S$, including compaction For $\delta \rightarrow \infty$ and $\mu_{c} \rightarrow 0$, BKS has the following form:

$$
\begin{aligned}
& \frac{\partial}{\partial t}\left(\phi_{s} \rho\right)+\frac{\partial}{\partial x}\left(\phi_{s} \rho v\right)=C \\
& \frac{\partial}{\partial t}\left(\phi_{g} \rho\right)+\frac{\partial}{\partial x}\left(\phi_{g} \rho g\right)=-C \\
& \frac{\partial}{\partial t}(\rho v)+\frac{\partial}{\partial x}\left(p+\rho v^{2}\right)=0
\end{aligned}
$$

where $\rho=\phi_{s} \rho_{s}+\phi_{g} \rho_{g} ; p=\phi_{s} p_{s}+\phi_{g} p_{g}-$

$$
\begin{aligned}
& \frac{\partial}{\partial t}\left(\phi_{s} \rho_{s} e_{s}\right)+\frac{\partial}{\partial x}\left(\phi_{s} \rho_{s} e_{s} v\right)+\phi_{s} p_{s} \frac{\partial v}{\partial x} \\
&+p_{g}\left(\frac{\partial \phi_{s}}{\partial t}+v \frac{\partial \phi_{s}}{\partial x}\right)=C\left(e_{s}+\frac{p_{g}}{\rho_{s}}\right)-H\left(T_{s}-T_{g}\right) \\
& \frac{\partial}{\partial t}\left(\phi_{g} \rho_{g} e_{g}\right)+\frac{\partial}{\partial x}\left(\phi_{g} \rho_{g} e_{g} v\right)+\phi_{g} p_{g} \frac{\partial v}{\partial x} \\
&-p_{g}\left(\frac{\partial \phi_{s}}{\partial t}+v \frac{\partial \phi_{s}}{\partial x}\right)=C\left(-e_{s}-\frac{p_{g}}{\rho_{s}}\right)+H\left(T_{s}-T_{g}\right) \\
& p_{s}-p_{g}-\beta\left(\phi_{s}\right)=0 .
\end{aligned}
$$

Before computing the characteristics, these equations need to be recast in the form

$$
U_{t}+\mathcal{A} U_{x}=R
$$

We shall take to be the s-vector

$$
U=\left\langle\phi_{s} \rho_{s}, \phi_{g} \rho_{g}, v, p_{s}, \phi_{s}\right\rangle \text {. }
$$


2.

Note that since (6) can be thought of as the definition of $p_{g}$, the system is effectively $s^{\text {th }}$ order.

Write (1.1) and (1.2) as

$$
\begin{aligned}
& \frac{\partial}{\partial t}\left(\phi_{s} \rho_{s}\right)+v \frac{\partial}{\partial x}\left(\phi_{s} \rho_{s}\right)+\phi_{s} \rho_{s} \frac{\partial v}{\partial x}=C \\
& \frac{\partial}{\partial t}\left(\phi_{g} \rho_{g}\right)+v \frac{\partial}{\partial x}\left(\phi_{g} \rho_{g}\right)+\phi_{g} \rho \frac{\partial v}{\partial x}=-C
\end{aligned}
$$

Mixture mass equation, $\frac{\partial \rho}{\partial t}+\frac{\partial}{\partial x}(f v)=0$, seduces (1,3) to

$$
\rho \frac{\partial v}{\partial t}+\rho v \frac{\partial v}{\partial x}+\frac{\partial p}{\partial x}=0 \text {. }
$$

With $p=\phi_{s} p_{s}+\left(1-\phi_{s}\right)\left(p_{s}-\beta_{s}\right)=-\left(1-\phi_{s}\right) p_{s}$ the above reduces further to

$$
\frac{\partial v}{\partial t}+v \frac{\partial v}{\partial x}+\frac{1}{\rho} \frac{\partial p_{s}}{\partial x}+\left\{\frac{\beta}{\rho}-\frac{\beta^{\prime}\left(\phi_{s}\right)}{\rho}\right\} \frac{\partial \phi_{s}}{\partial x}=0
$$

Consider now the solid energy eau. (1.4). Witt

$$
(i)=\frac{\partial}{\partial t}()+v \frac{\partial}{\partial x}() \text {. }
$$

it can be rearranged as (upon using (1.1))

$$
\dot{e}_{s}+\frac{p_{s}}{P_{s}} v_{c}+\frac{p_{s}}{\phi_{s} P_{s}} \dot{\phi}_{s}=\frac{L}{\phi_{s} P_{s}}\left[\frac{p_{g}}{P_{s}} C-H\left(T_{s}-T_{g}\right)\right]
$$

Now, with $e_{s}=e_{s}\left(p_{s}, \rho_{s}\right)$,

$$
\begin{aligned}
& \dot{p}_{s}=\frac{1}{\partial e_{s} / \partial p_{s}}\left[\dot{e}_{s}-\frac{\partial e_{s}}{\partial \rho_{s}} \dot{\rho}_{s}\right] \\
& =\frac{1}{\partial e_{s} / \partial p_{s}}\left[-\frac{p_{s}}{\rho_{s}} v_{x}-\frac{p_{g}}{\phi_{s} p_{s}}-\frac{\partial e_{s}}{\partial p_{s}}\left\{-\rho_{s} \phi_{s}-\phi_{s} \rho_{s} v_{x}+C\right\}\right. \\
& \left.+\frac{1}{s}\left\{\frac{1}{s}-H\left(T_{s}-T_{g}\right)\right\}\right]
\end{aligned}
$$




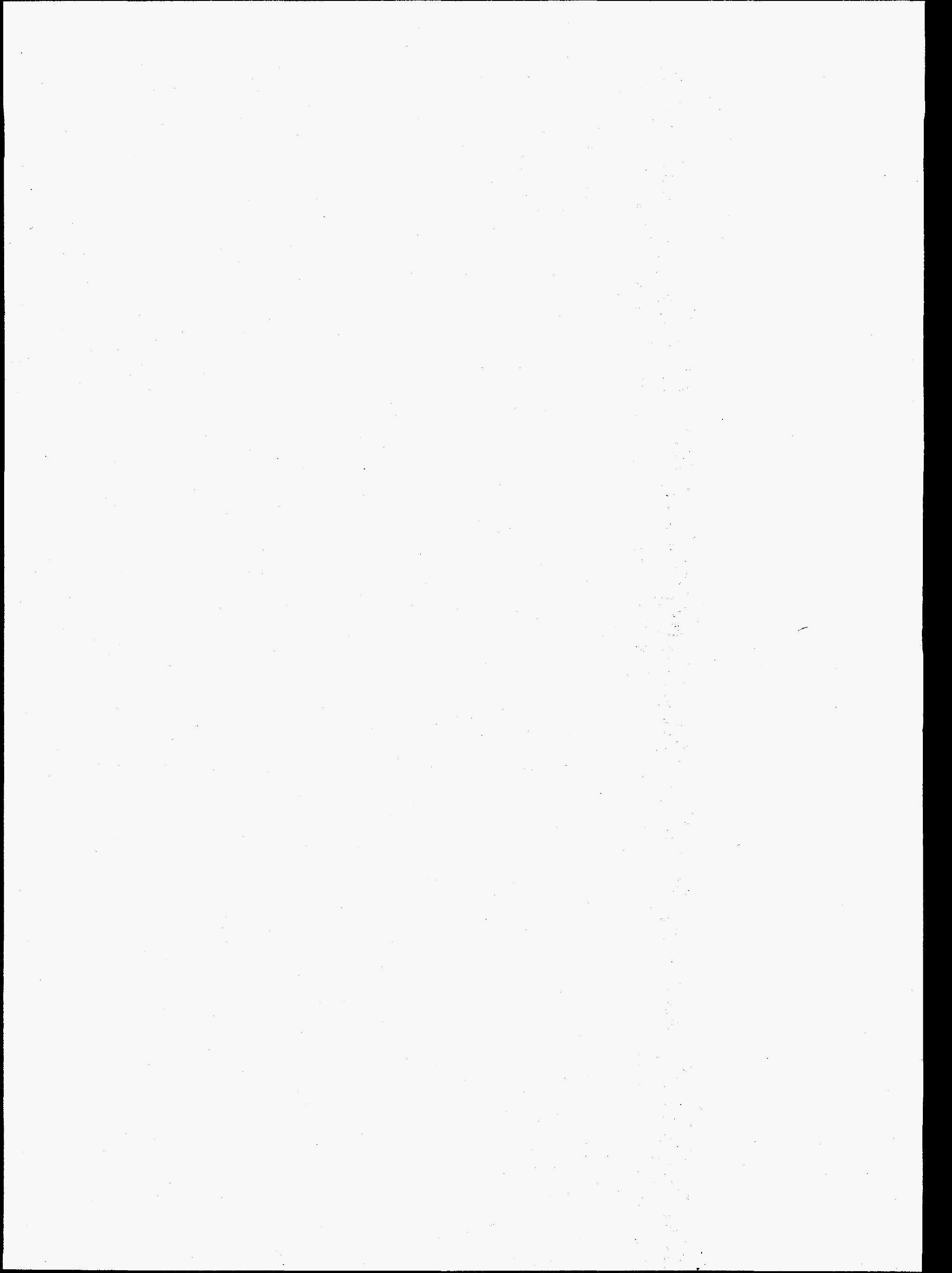


Recall that

$$
\rho_{s} c_{s}^{2}=\frac{1}{\partial e_{s} / \partial p_{s}}\left[\frac{p_{s}}{\rho_{s}}-\rho_{s} \frac{\partial e_{s}}{\partial \rho_{s}}\right]
$$

Thus,

$$
\begin{aligned}
\dot{p}_{s}= & -\rho_{s} c_{s}^{2} \frac{\partial v}{\partial x}+\frac{1}{\phi_{s}} \frac{\dot{\phi}_{s}}{\partial e_{s} / \partial p_{s}}\left[\rho_{s} \frac{\partial e_{s}}{\partial \rho_{s}}-\frac{p_{s}}{\rho_{s}}+\frac{p_{s}-p_{s}}{\rho_{s}}\right] \\
& -\frac{\partial e_{s} / \partial \rho_{s}}{\partial e_{s} / \partial p_{s}} \frac{c}{\phi_{s}}+\frac{1}{\phi_{s} \rho_{s}}\left[\frac{p_{s}}{\rho_{s}} C-H\left(T-T_{s}\right)\right] \frac{1}{\partial e_{s} / \partial p_{s}}
\end{aligned}
$$

or,

$$
\begin{aligned}
& \dot{p}_{s}+p_{s} c_{s}^{2} \frac{\partial v}{\partial x}+\frac{\phi_{s}}{\phi_{s}}\left[\rho_{s} c_{s}^{2}-\frac{1}{\partial e_{s} / \partial p_{s}} \frac{p_{s}-p_{s}}{p_{s}}\right]
\end{aligned}
$$

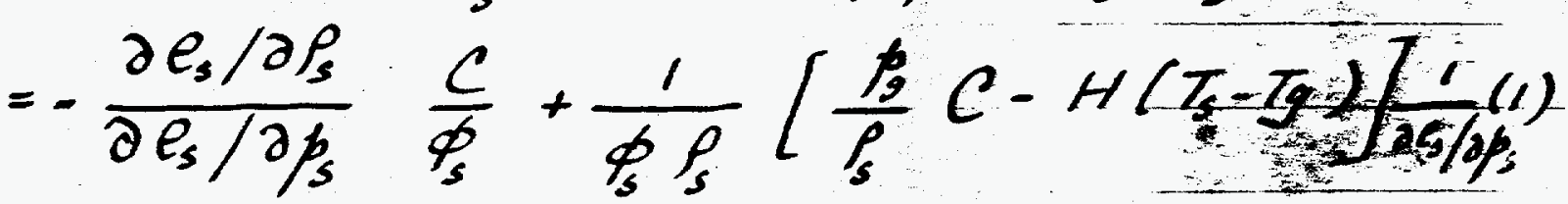

We now turn to the gas energy eau. (1.5). Proceeding as with the solid energy,

$$
\begin{aligned}
& \dot{e}_{g}+\frac{p_{g}}{P_{g}} V_{x}-\frac{P_{g}}{\phi_{g} P_{g}} \dot{\phi}_{s}=\frac{1}{\phi_{g} P_{g}}\left[-\frac{P_{g}}{P_{s}} C+H\left(T_{s}-T_{g}\right)\right](2 \\
& \dot{p}_{g}=\frac{1}{\partial e_{g} / \partial \rho_{g}}\left[\dot{e}_{g}-\frac{\partial e_{g}}{\partial \rho_{g}} \dot{\rho}_{g}\right] \\
& =\frac{1}{\partial e_{g} / \partial p_{g}}\left[-\frac{p_{g}}{P_{g}} V_{x}+\frac{p_{g}}{\phi_{g} \rho_{g}} \dot{\phi}_{s}-\frac{\partial e_{g}}{\partial P_{g}} \frac{1}{\phi_{g}}\left\{+\rho_{g} \dot{\phi}_{s}\right.\right. \\
& \left.\left.-\phi_{g} \rho_{g} v_{x}-C\right\}+\frac{1}{\phi_{g} P_{g}}\left\{-\frac{\phi_{g}}{\rho_{s}} C+H\left(T_{s}-T_{g}\right)\right\}\right] \\
& =-\rho_{g} c_{g}^{2} v_{x}+\frac{\phi_{s}}{\phi_{g}} \frac{1}{\partial e_{g} / \partial p_{g}}\left\{\frac{p_{g}}{\rho_{g}}-\rho_{g} \frac{\partial e_{g}}{\partial \rho_{g}}\right\} \\
& +\frac{1}{\partial e_{g} / \partial p_{g}}\left[+\left(\partial \rho_{g} / \partial P_{g}\right) \frac{C}{\phi_{g}}+\frac{1}{\phi_{g} \rho_{g}}\left\{-\frac{p_{g}}{P_{g}} C+H\left(T_{s}-T_{g}\right)\right\}\right]
\end{aligned}
$$




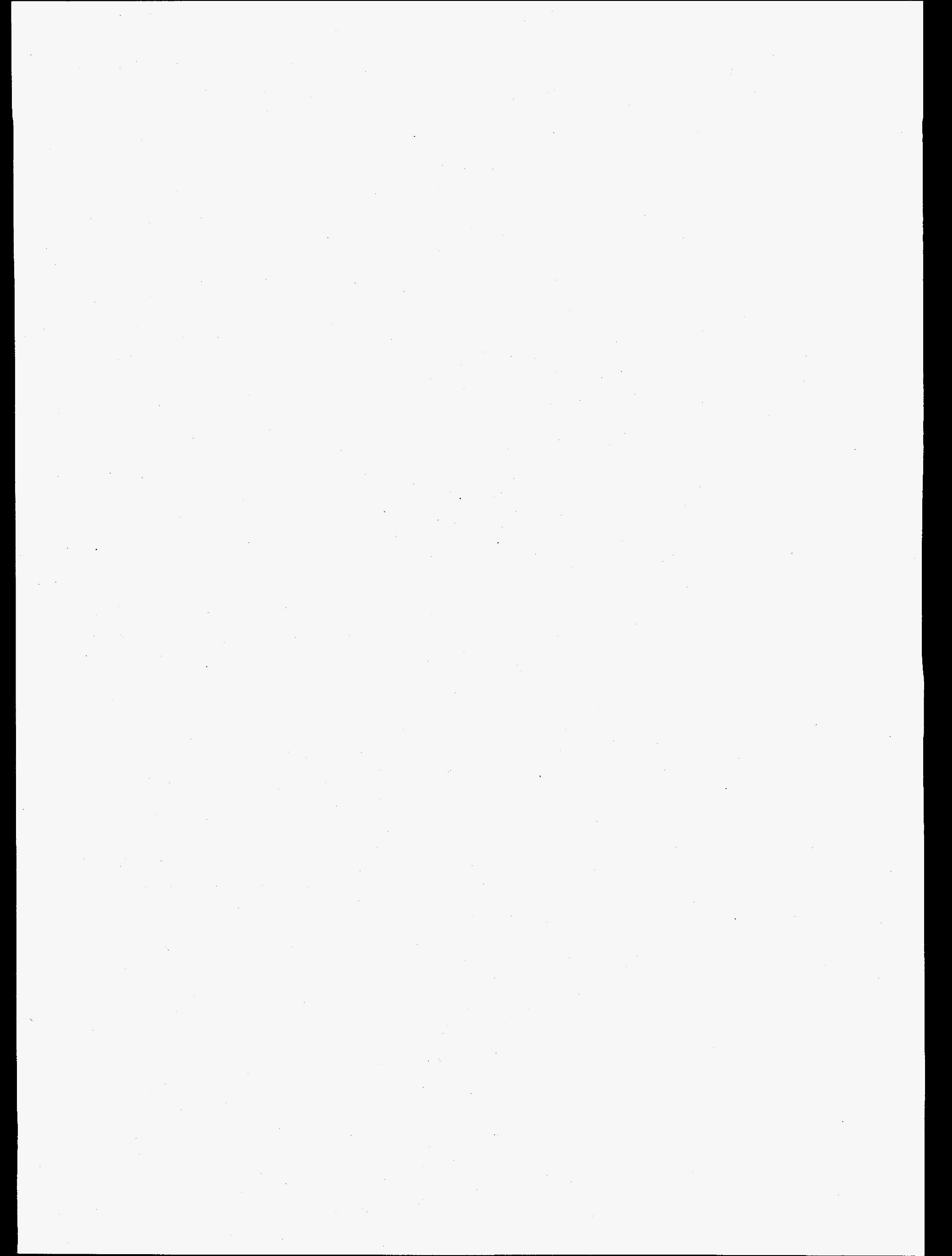


or,

$$
\begin{aligned}
& \dot{p}_{g}+\hat{y}_{y}^{2} v_{x}+\frac{\dot{\phi}}{\phi_{y}}\left\{-\rho_{g} G_{g}^{2}\right\} \\
& =\frac{1}{\partial \mathcal{E}_{g} / \partial f_{g}}\left[\frac{\mathcal{C}}{g} \frac{\partial e_{g}}{\partial P_{g}}+\frac{1}{F_{g} P_{g}}\left\{-\frac{p_{g}}{P_{s}} C+H\left(T-T_{g}\right)\right\}\right]
\end{aligned}
$$

Adding (3.1) and -(4.1),

$$
\begin{aligned}
& \dot{p}_{s}-\dot{p}_{g}+\left(\rho_{s} c_{s}^{2}-\rho_{g} C_{g}^{2}\right) v_{x}+\dot{\phi}_{s}\left[\frac{\rho_{s} C_{s}^{2}}{\phi_{s}}+\frac{\rho_{g} C_{g}^{2}}{\phi_{g}}\right. \\
& \left.-\frac{1}{\partial e_{s} / \partial p_{s}} \frac{p_{s}-p_{g}}{\phi_{s} \rho_{s}}\right]=\frac{1}{\phi_{s} \partial e_{s} / \partial p_{s}}\left[-\frac{\partial e_{s}}{\partial P_{s}} C\right. \\
& \left.+\frac{1}{\rho_{s}}\left\{\frac{p_{s}}{P_{s}} C-H\left(T_{s}-T_{g}\right)\right\}\right]-\frac{1}{\phi_{g} \partial e_{g} / \partial p_{g}}\left[\frac{\partial e_{s}}{\partial P_{g}} C\right. \\
& \left.+\frac{1}{\rho_{g}}\left\{-\frac{p_{g}}{\rho_{s}} C+H\left(T_{s}-T_{g}\right)\right\}\right] \equiv \mathcal{R}_{s g}, \text { say. }
\end{aligned}
$$

with $p_{s}-p_{g}=\beta_{s}\left(\phi_{s}\right)$, the above becomes

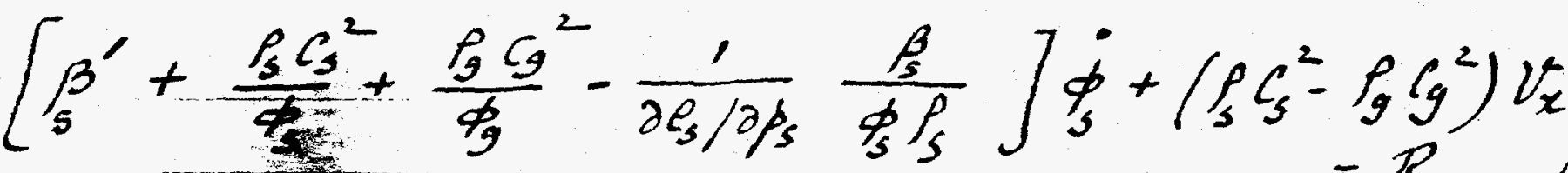

$$
\begin{aligned}
& =\mathcal{R}_{s g}
\end{aligned}
$$

Emus. $(2.1),(2.2),(2.3),(3.1)$ and $(4.3)$ now constitute the recast $5^{*}$-order system e.

with

$$
A \equiv \frac{\rho_{s} C_{s}^{2}}{\phi_{s}}+\frac{\rho_{S} \mathcal{C}_{g}^{2}}{\phi_{g}}+\beta_{s}^{\prime}-\frac{1}{\partial e_{s} / \partial p_{s}} \frac{\beta_{s}}{\phi_{s} \rho_{s}},
$$

and $B \equiv \frac{1}{\phi}\left[P_{s} C_{S}^{2}-\frac{1}{\partial e_{S} / \partial p_{s}} \frac{\beta_{s}}{\rho_{S}}\right]$, 


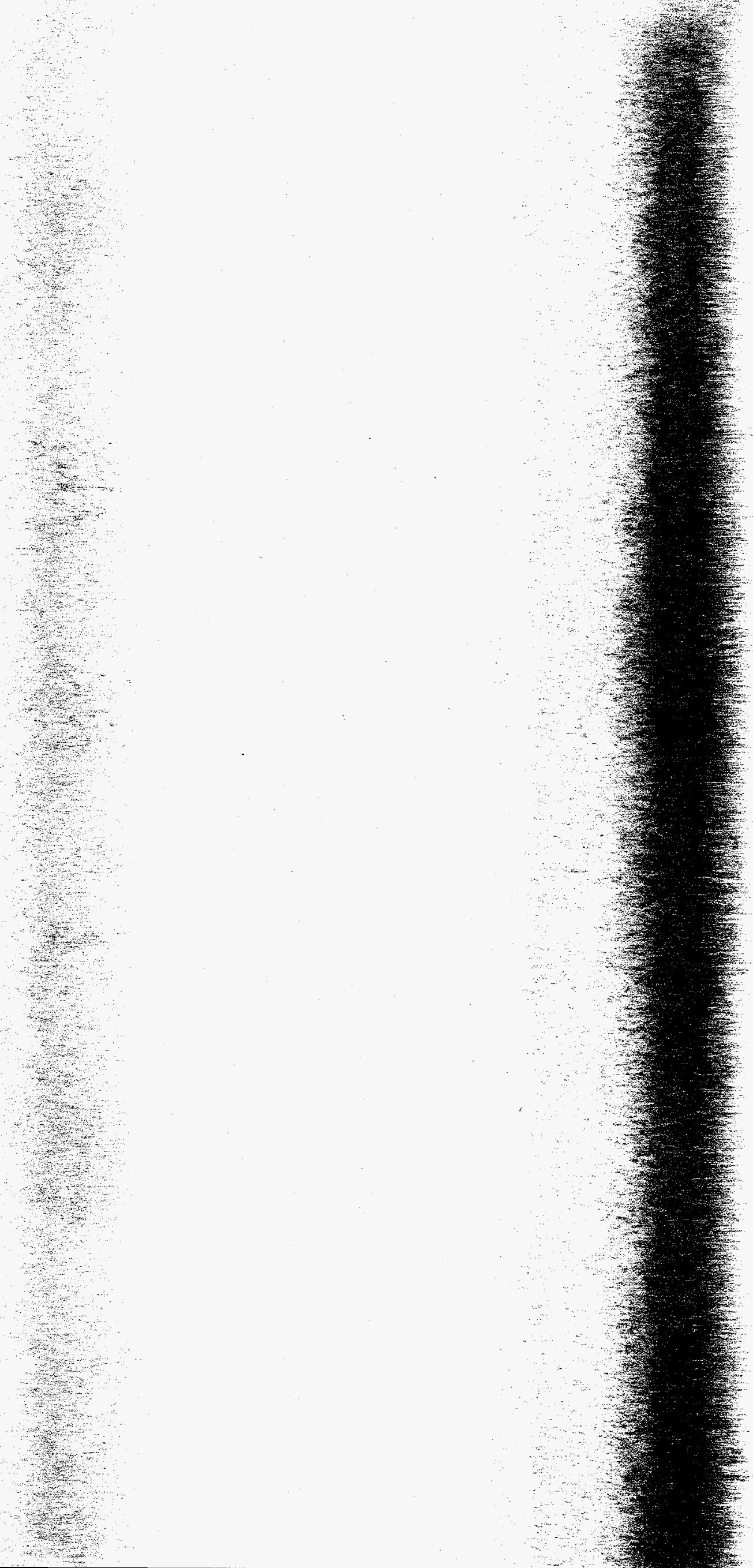


the syst, becomes

$$
\begin{aligned}
& \frac{\partial}{\partial t}\left(\phi_{s} f\right)+v_{\partial} \frac{\partial}{\partial x}\left(\phi_{s} f_{s}\right)+\phi_{s} P_{s} v_{x}=t \\
& \frac{\partial}{\partial t}\left(\phi_{g} f_{g}\right)+v_{\partial x} \frac{\partial}{\partial x}\left(\phi_{g} P_{g}\right)+\phi_{g} f_{g} v_{x}= \\
& \frac{\partial v}{\partial t}+v \frac{\partial v}{\partial x}+\frac{1}{\rho} \frac{\partial p_{s}}{\partial x}+\frac{\beta-\phi \beta^{\prime}}{\rho} \frac{\partial}{\partial x} \\
& \frac{\partial p_{s}}{\partial t}+v \frac{\partial p_{s}}{\partial x}+\rho_{s} C_{s}^{2} v_{x}+\mathcal{B}\left(\frac{\partial \phi_{s}}{\partial t}+y_{t}\right. \\
& \frac{\partial \phi_{s}}{\partial t}+v_{t} \frac{\partial \phi_{s}}{\partial x}+\frac{P_{s} c_{s}^{2}-P_{g} c_{g}^{2}}{A} v_{x}=
\end{aligned}
$$

The 44 gfy above may be rewrittur

$$
\frac{\partial p_{s}}{\partial t}+y_{x} \frac{\partial p_{s}}{\partial x}+v_{x}\left[P_{s} C_{s}^{2}-\frac{B}{A}\left(P_{s} C_{s}^{2}\right.\right.
$$

vote that

$$
\begin{aligned}
& A 3+\frac{P_{g} C_{g}^{2}}{\phi_{g}}+\beta^{\prime}
\end{aligned}
$$

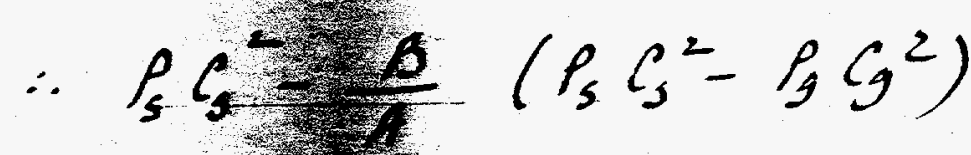

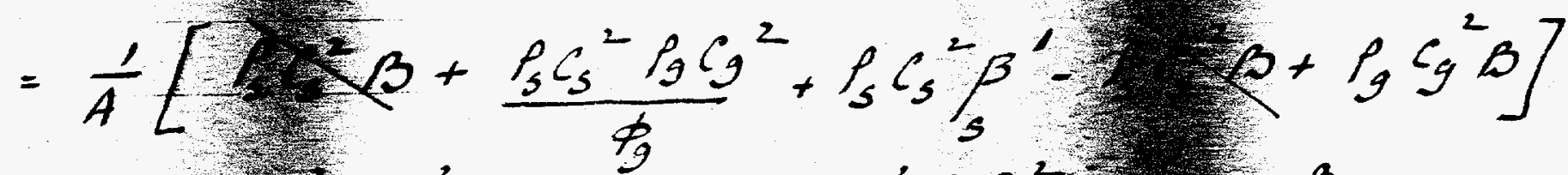

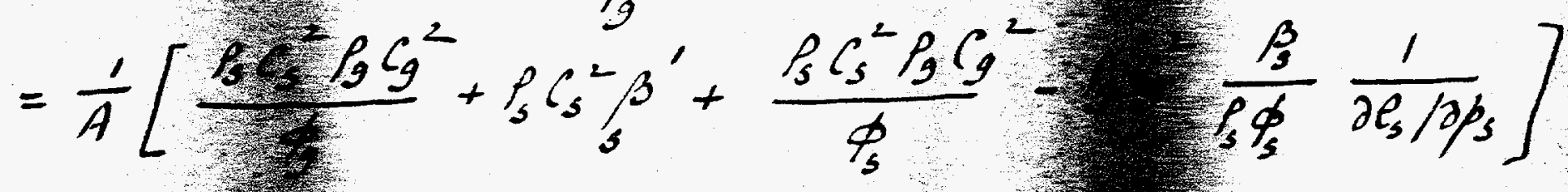

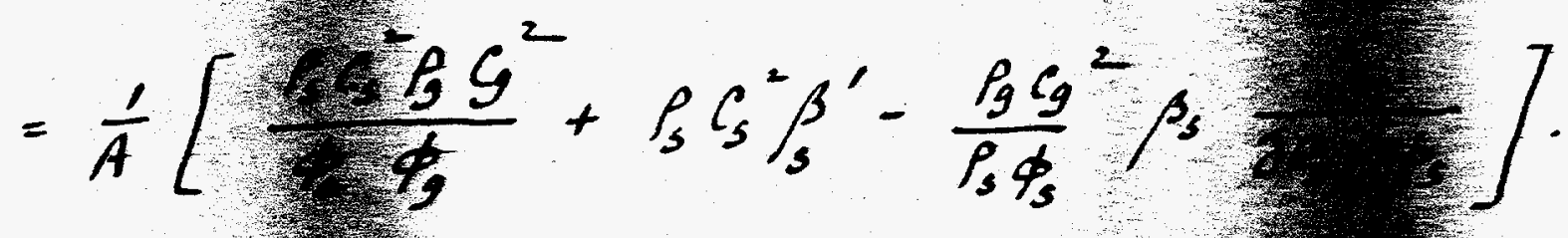

$$
\begin{aligned}
& \equiv \mathcal{C} / A \text {, say. }
\end{aligned}
$$


6.

The system e now takes the forme $\underline{U}_{t}+\underline{A} \underline{U}_{1}=\ldots$ whine

$$
A=\left[\begin{array}{ccccc}
v & 0 & \phi_{s} \rho_{s} & 0 & 0 \\
0 & v & \phi_{g} \rho_{g} & 0 & 0 \\
0 & 0 & v & \frac{1}{\rho} & \frac{\rho_{s}-\phi_{g s}}{\rho} \\
0 & 0 & \frac{C}{A} & v & 0 \\
0 & 0 & \frac{\rho_{s} c_{s}^{2}-\rho_{g} C_{g}^{2}}{A} & 0 & v
\end{array}\right]
$$

The eigenvalues are given by

$$
\begin{aligned}
& (v-\lambda)^{2}\left[-\frac{\rho_{s} C_{s}^{2}-\rho_{g} G^{2}}{A}(v-\lambda) \frac{\beta_{s}-\phi_{g} \beta_{s}^{\prime}}{\rho}+(v-\lambda)\left[(v-\lambda)^{2}-\frac{C}{A \rho}\right\}\right]=0 \\
& (v-\lambda)^{3}\left[(v-\lambda)^{2}-\frac{C}{A \rho}-\frac{\left(\rho_{s} c_{s}^{2}-\rho_{g} \rho_{g}^{2}\right)\left(\beta_{s}-\rho_{g} \beta_{s}^{\prime}\right)}{A \rho}\right]=0 \\
& \text { wider } D^{2} \equiv \\
& {\left[C+\left(\rho_{s} C_{s}^{2}-\rho_{g} C_{g}^{2}\right)\left(\beta_{s}-\phi_{g} \rho_{s}^{\prime}\right)\right]} \\
& =\left[\frac{\rho_{s} C_{s}^{2} \rho_{g} C_{g}^{2}}{\phi_{s} \phi_{g}}+\rho_{s} C_{s}^{2} \beta_{s}^{\prime}-\frac{\rho_{g} C_{g}^{2}}{\rho_{s} \phi_{s}} \beta_{s} \frac{1}{\partial \rho_{s} / \partial \rho_{s}}-\phi_{g} \beta_{s}^{\prime}\left(\rho_{s} C_{s}^{2}-\rho_{g} C_{g}^{2}\right)\right. \\
& \left.+\beta_{s}\left(P_{s} C_{s}^{2}-P_{g} C_{g}^{2}\right)\right] \frac{1}{A P} \\
& \frac{1}{P P}\left\{\frac{\rho_{s} C_{s}^{2}+P_{g} G_{g}^{2}}{\phi_{s} \phi_{g}}+\beta_{s}^{\prime}\left(\rho_{s} \varphi_{s} C_{s}^{2}+\rho_{g} \phi_{g} G^{2}\right)+\beta\left[P_{s} C_{s}^{2}-\rho_{g} G^{2}-\frac{P_{s} g^{2}}{\rho_{s} \rho_{s} \partial P_{s} / \partial p_{s}}\right]\right\}
\end{aligned}
$$




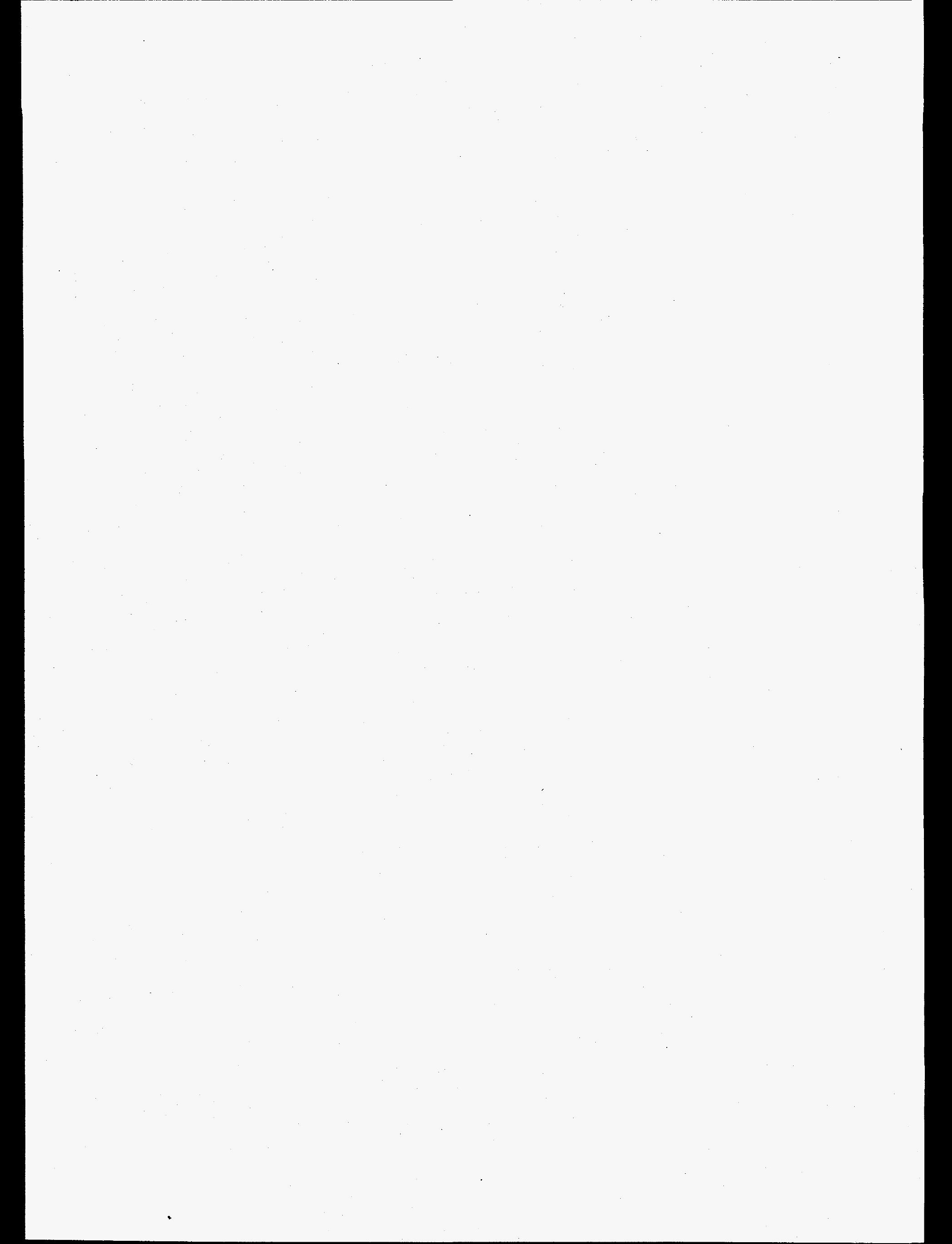




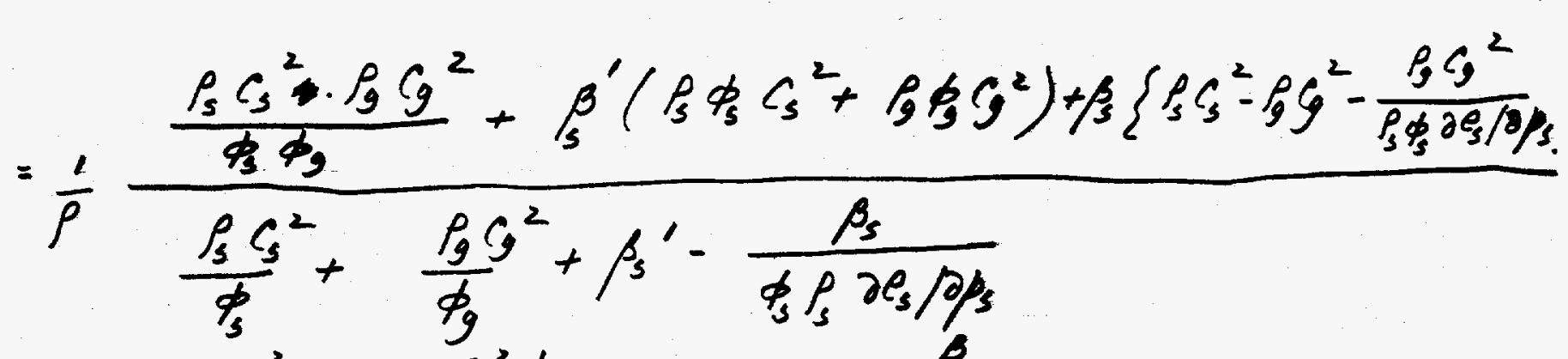

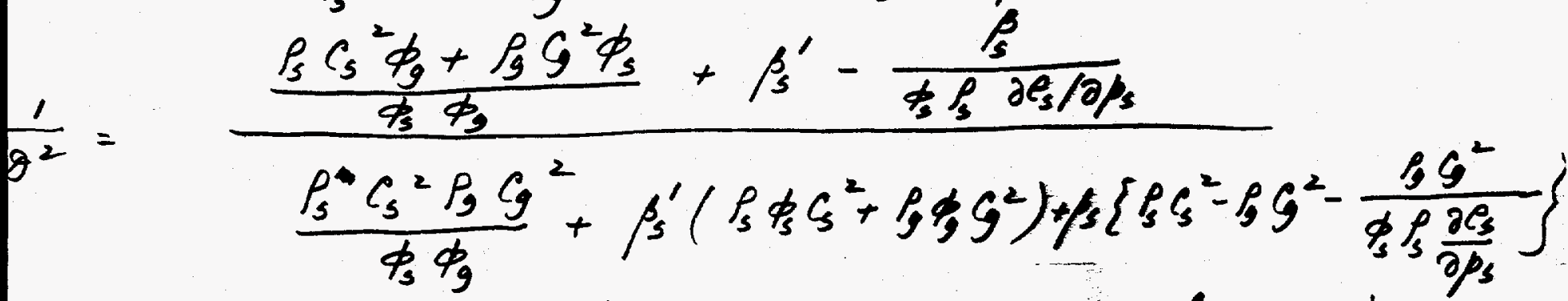

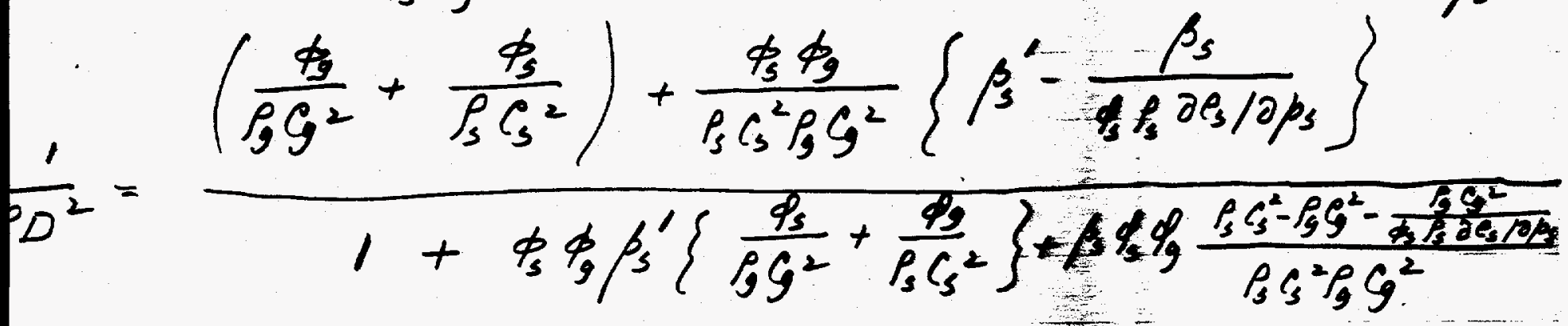

Foe $\beta_{s}=\beta_{s}^{\prime}=0$, get

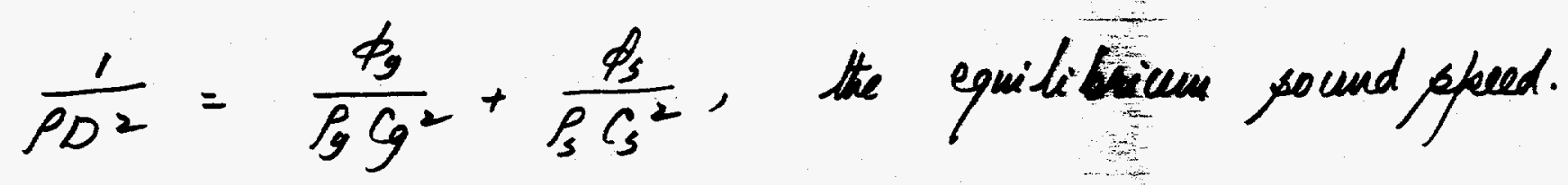

The eigenvalues are, now,

$$
\lambda=v, v, v, \quad v \pm d .
$$



ITEM 5 
$+2$

ㄱ.

3

. 
Eau. of state for solid:

$$
\begin{aligned}
e_{s} & =-T_{3}\left(1-\frac{P_{s 0}}{P_{s}}\right)+T_{4}\left\{\left(\frac{P_{s}}{P_{0}}\right)^{n-1}-(n-1)\left(1-\frac{P_{00}}{P_{s}}\right)-1\right\} \\
& +\frac{1}{G} \frac{P_{s 0}}{P_{s}}\left[P_{s}-P_{s 0}-\frac{A_{3}}{n}\left\{\left(\frac{P_{1}}{l_{30}}\right)^{n}-1\right\}\right]
\end{aligned}
$$

Units for $e$ are ers $/ 9 m$.

$$
\begin{aligned}
& \frac{n=9.8}{T(\text { es } / g m)}=\frac{C_{v s} T_{s 0} G}{f_{s 0}} \\
& T_{4}(\text { enos } / g m)=\frac{A_{s}}{P_{s o} n(n-1)} \\
& G\left(9 \mathrm{~m} / \mathrm{cm}^{3}\right)=2.1 \\
& \left.A_{0} \text { (ayes } / \mathrm{cm}^{2}\right)=1.35 \times 10^{\prime \prime} \\
& T_{s 0}=300^{\circ} \mathrm{K}, \quad R_{s 0}=10^{6} \text { dynes } / \mathrm{cm}^{2} \\
& C_{\text {vs }}\left(\text { ergs } / g^{m}{ }^{\circ} \mathrm{K}\right)=15 \times 10^{6} \\
& C_{v 3}\left(T_{s}-T_{s 0}\right)=e_{s}+T_{3}\left(\frac{P_{s}}{P_{s 0}}-1\right) \frac{\rho_{s 0}}{P_{s}} \\
& -T_{4}\left\{\left(\frac{P_{5}}{P_{10}}\right)^{n-1}-(n-1)\left(1-\frac{P_{30}}{P_{5}}\right)-1\right\}
\end{aligned}
$$


Ign. of state for gas:

$$
\begin{aligned}
e_{g} & =-h_{\text {rd }}-C_{v g} T_{g o}+\frac{1}{\phi_{s_{0}} \rho_{S_{0}}}\left[\frac { \nu _ { g } } { \omega } \left\{P_{g}-A e^{-}\right.\right. \\
& \left.\left.-B e^{-R_{2} \nu_{g}}\right\}+\frac{A}{R_{1}} e^{-R_{1} \nu_{g}}+\frac{B}{R_{2}} e^{-R_{2} \nu_{g}}\right]
\end{aligned}
$$

Here,

$$
\nu_{g}=\frac{\phi_{s 0} P_{s o}}{\rho_{g}}
$$

$$
\begin{aligned}
& c_{v g}=\left[2.4-0.28\left(\phi_{s 0} \rho_{s 0}-1.3\right)\right] \times 10^{4} \text { es } / g \text { mo } \\
& T_{90}=300 \% \\
& R_{1}=4.2, \quad R_{2}=1.0, \omega=0.25 \\
& A\left(\text { dyaus } / \mathrm{cm}^{2}\right)=\left[-4.005+21.39 \phi_{s 0} \rho_{s 0}-16.23\left(\phi_{s 0} S_{s}\right.\right. \\
& \left.+4.595\left(\phi_{50} \mathrm{~S}_{50}\right)^{3}\right] \times 10^{12} \\
& \left.B \text { (dues } / \mathrm{cm}^{2}\right)=\left[-0.014+0.0349 \phi_{50} S_{30}\right. \\
& +0.0156\left(\$_{50} / 50\right)^{2}-0.026\left(\phi_{30} f_{50}\right)
\end{aligned}
$$

$h_{\text {nut }}=$ comet, adjured so that the undisturbed material has $g=0$. 
3.

$$
\begin{aligned}
\operatorname{Cvg}\left(T_{g}-T_{g 0}\right)= & C_{g}+h_{\text {ad }}-\frac{1}{\phi_{s_{0}} P_{s_{0}}}\left\{\frac{A}{R_{1}} e^{-R_{1} \nu_{g}}\right. \\
& \left.+\frac{\beta}{R_{2}} e^{-R_{2} \nu_{g}}\right\} .
\end{aligned}
$$




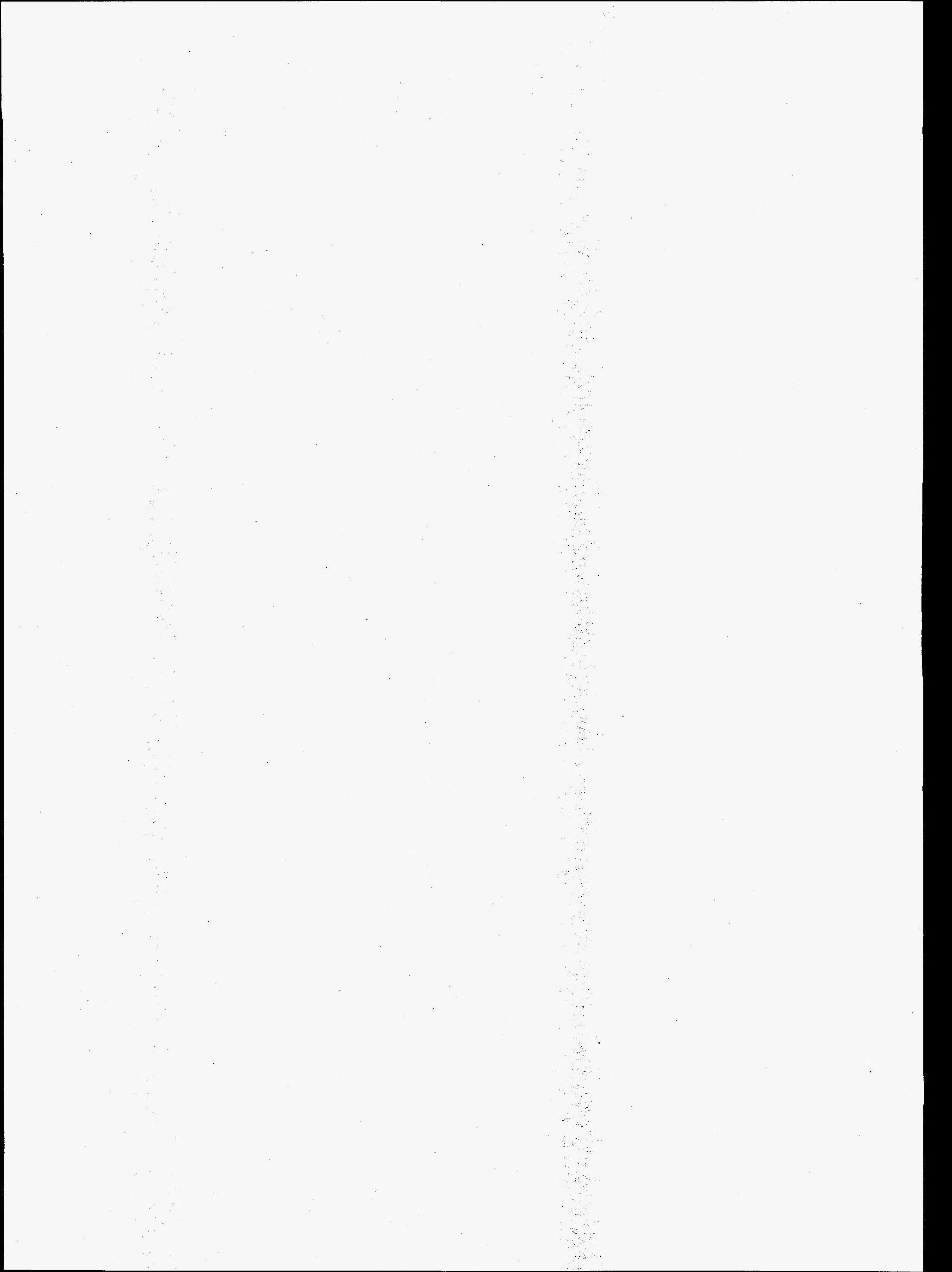




\section{Gas / Solid Velocity}

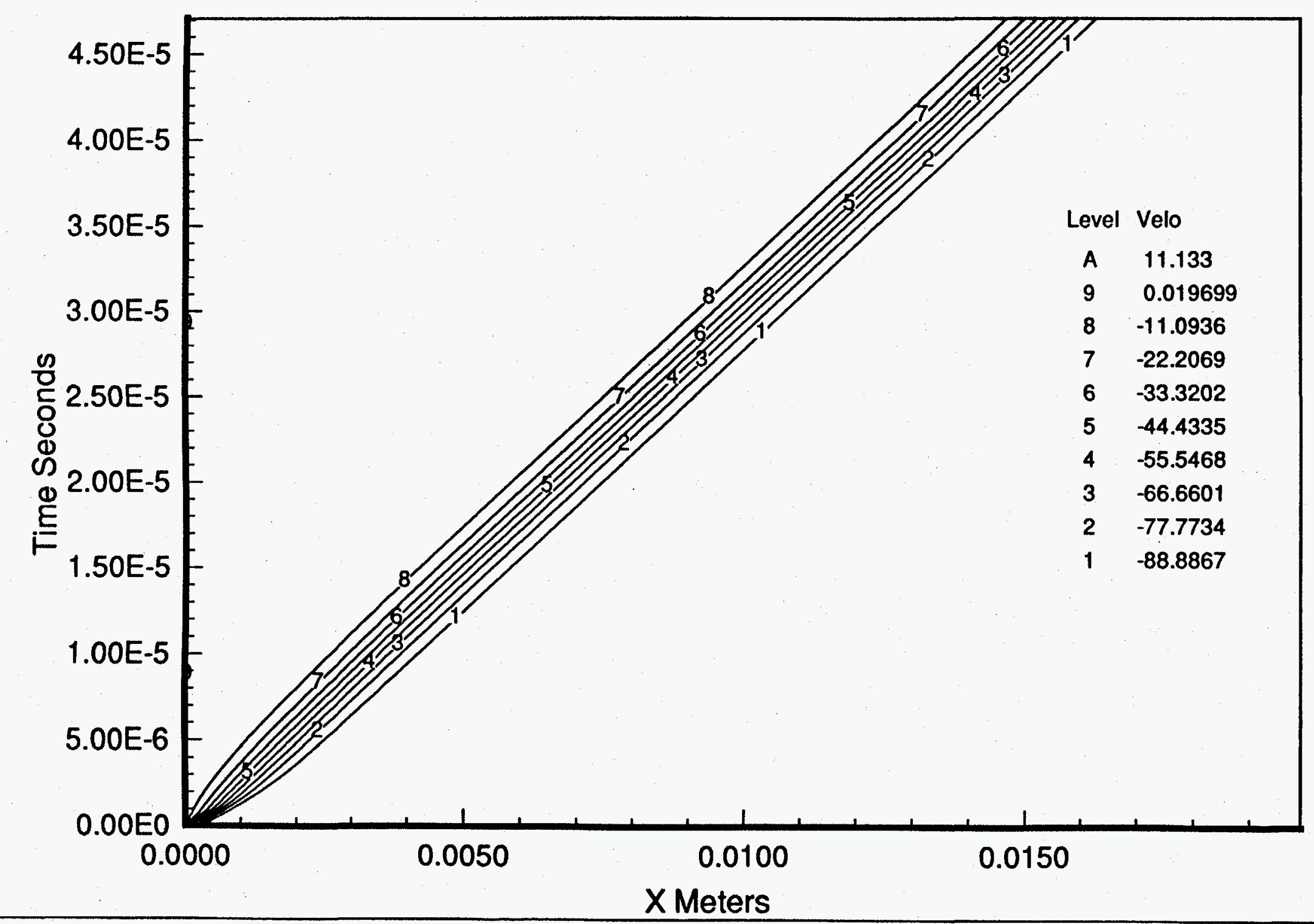


(2D) || Print || fort40.plt || 2-phase, single velocity, equal temp Compaction: PRASAD

\section{Mixture Density}

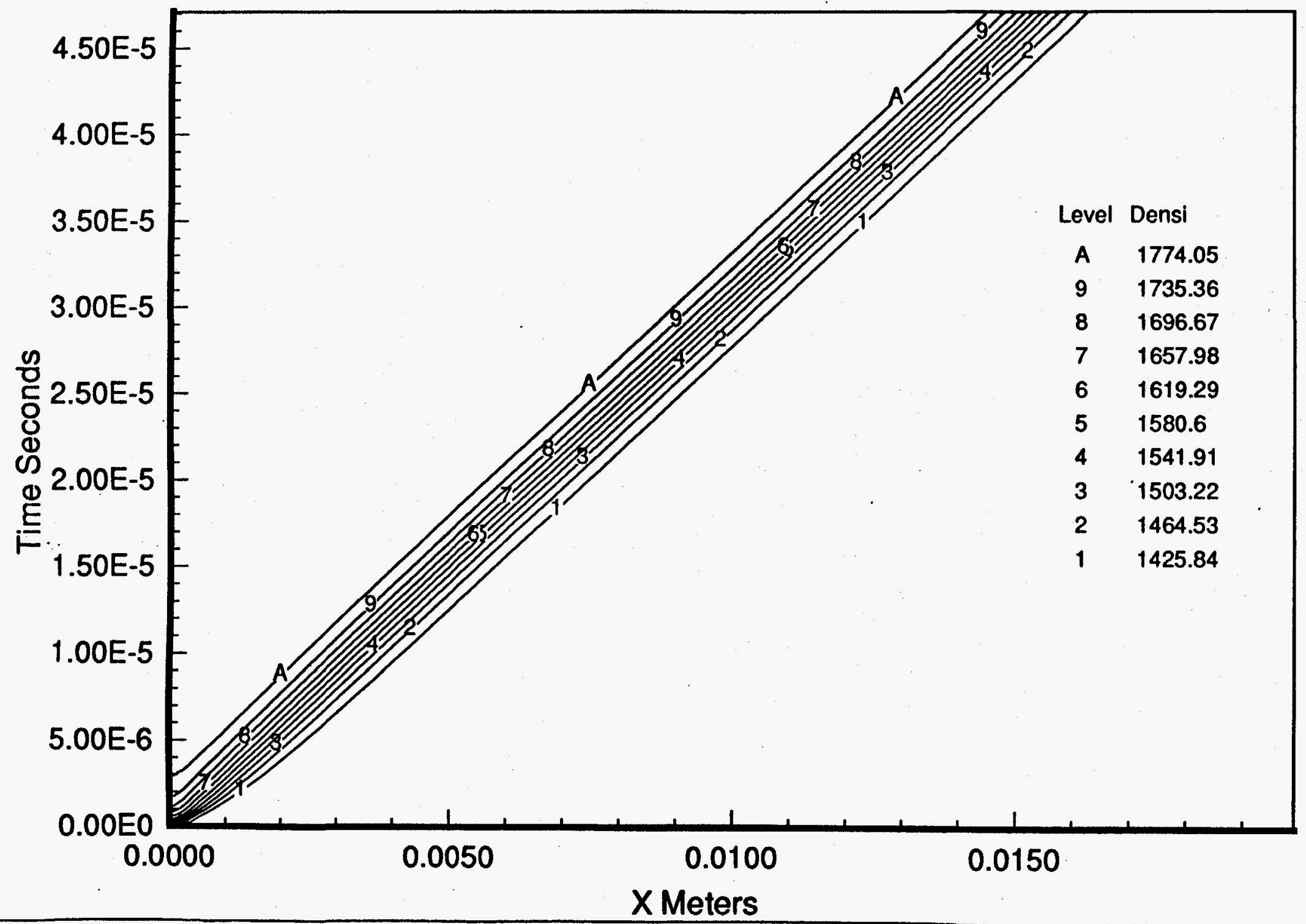




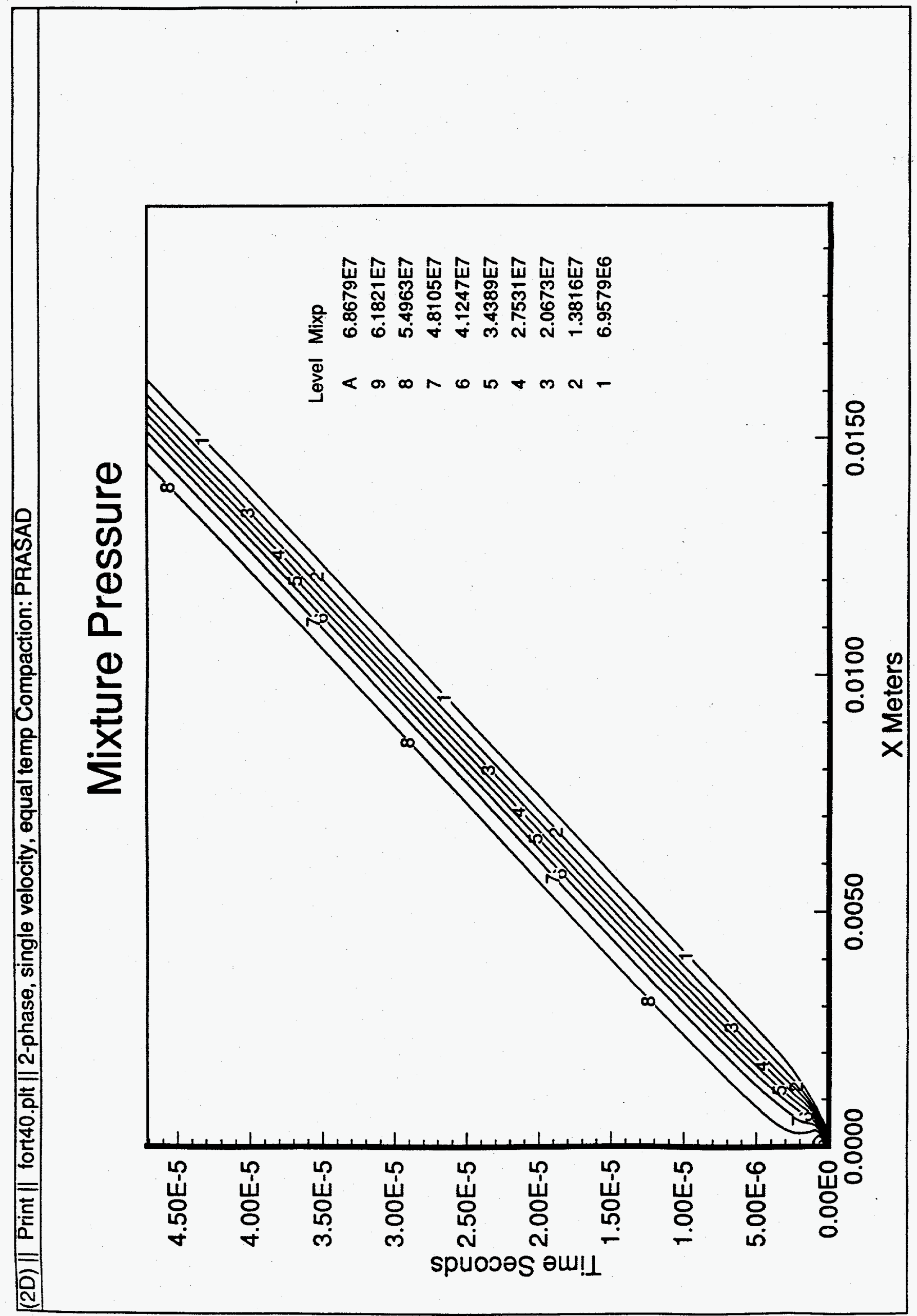




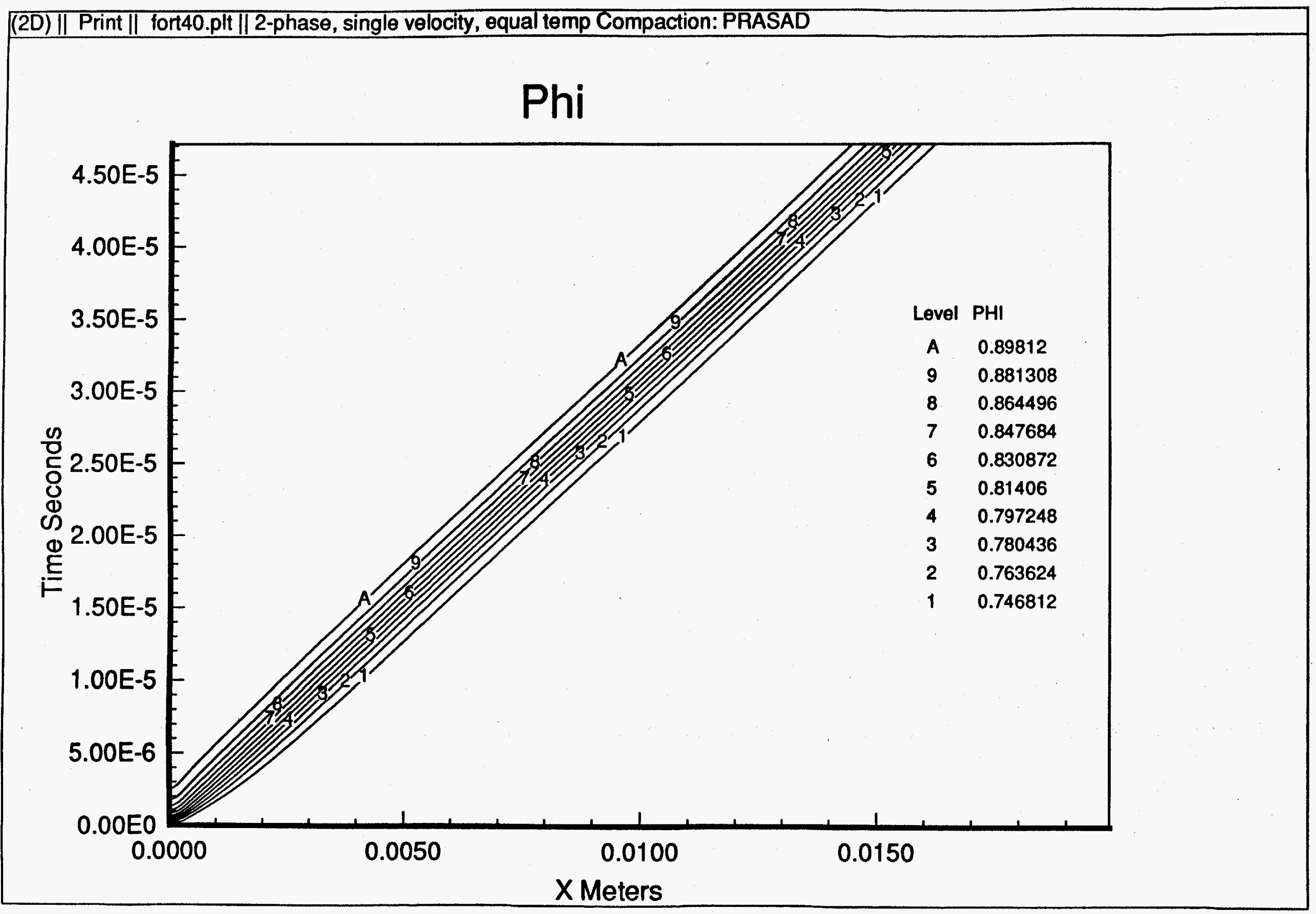




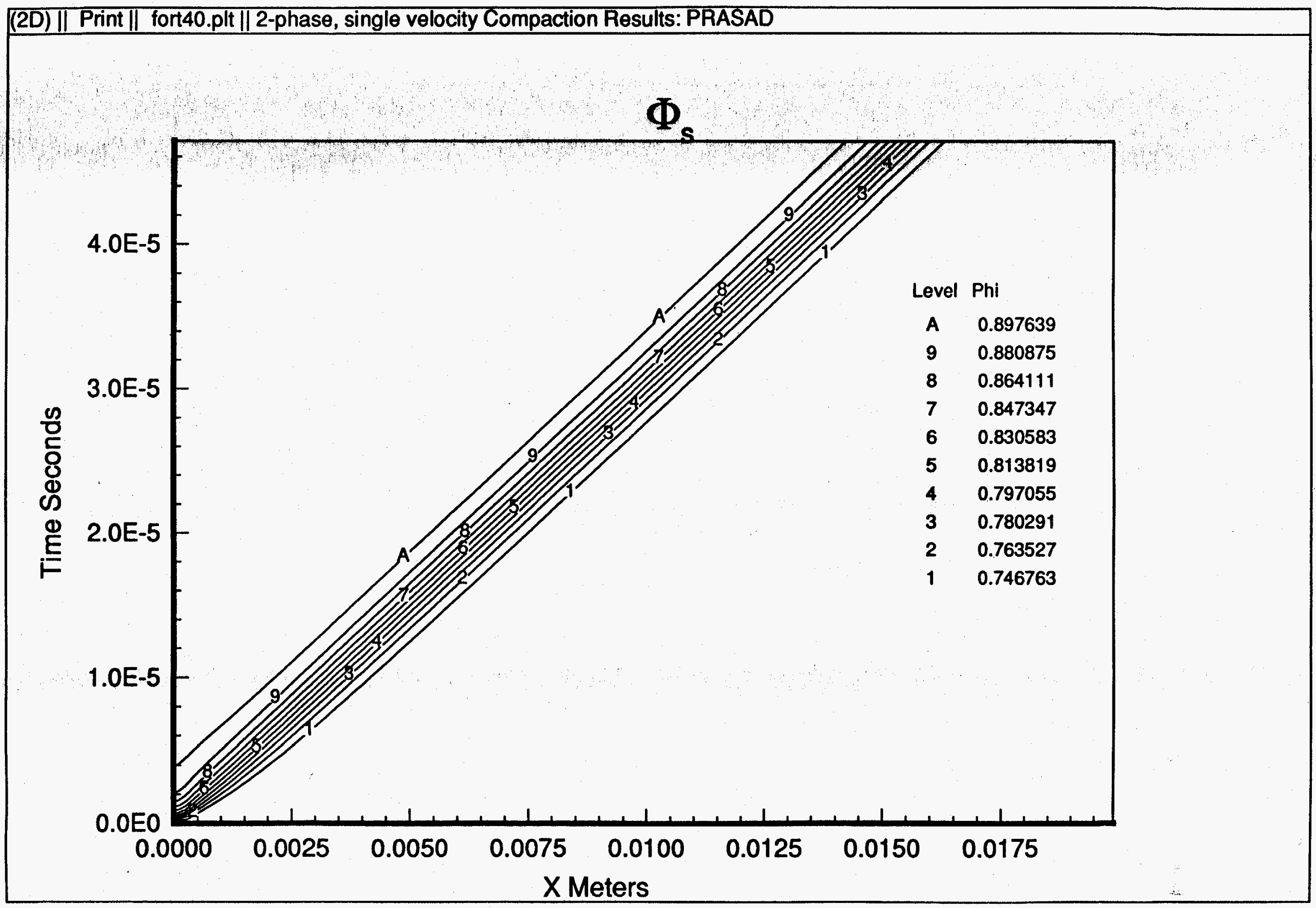




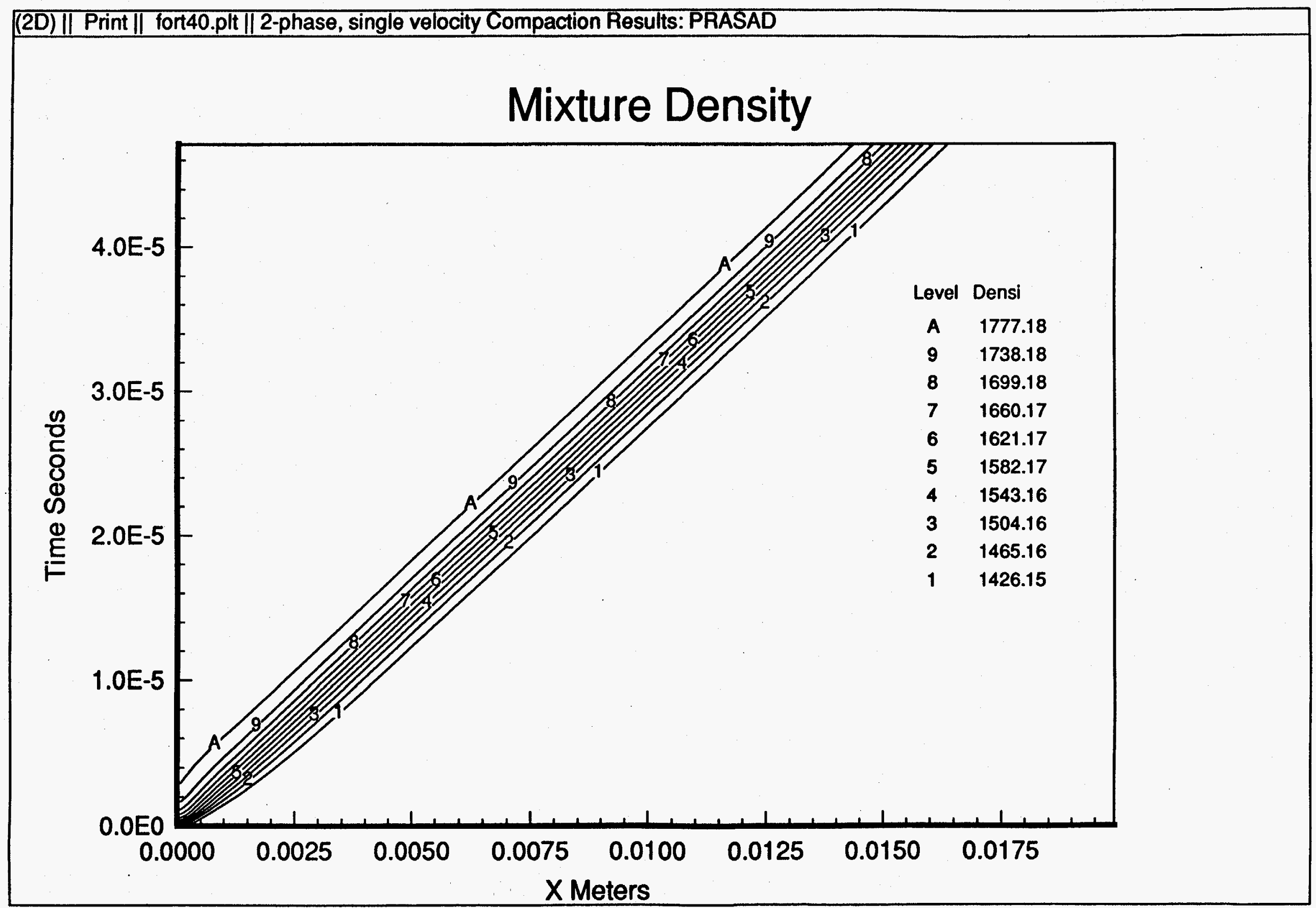




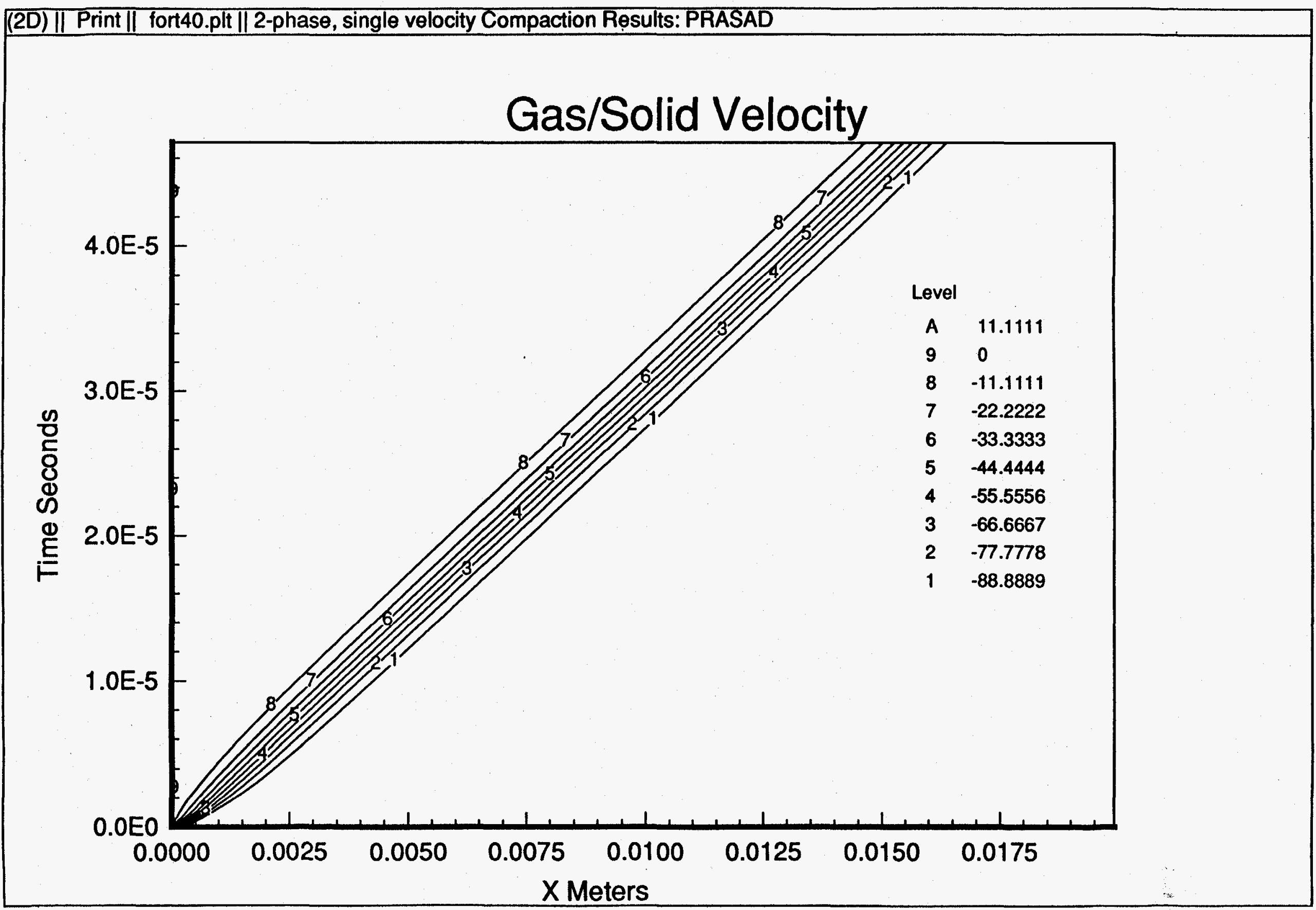





\section{ITEM 6}


on the 6-egn. equivelocity model

1. Advil has recently written down a 6-egn. model by starting with the 7-eqn. B-N model and setting $v_{s}=v_{g}$. Essentially the two individual momentum ecus. are replaced by a single overall momentum eqn; hence the reduction to a set of 6 equs.

Such a point of view seems inconsistent to me. In genre, the equal-velocity requirement will not agree with one of the momentum e equations that has been discarded. A rational scheme for deriving the equivelocity model would be to employ the limit $\delta \rightarrow \infty$. Then, one is nat discording any equation; simply replacing it by a reduced form. Further, one is recognizing the fact that mechanical equilibrium (vg = vs) does not necessarily imply absence of energy exchange between phases dire to drag.

As the following calculations show, the reduced model so obtained is nonconsenvative. 
(19)

The 6-eqn. equivelocity model, as derived from Bacr-Numziato

start with BN: Mass

$$
\begin{aligned}
& \frac{\partial}{\partial t}\left(\phi_{s} \rho_{s}\right)+\frac{\partial}{\partial x}\left(\phi_{s} \rho_{s} v_{s}\right)=C \\
& \frac{\partial}{\partial t}\left(\phi_{g} \rho_{g}\right)+\frac{\partial}{\partial x}\left(\phi_{g} \rho_{g} v_{g}\right)=-C
\end{aligned}
$$

Momentum

$$
\begin{gathered}
\frac{\partial}{\partial t}\left(\phi_{s} \rho_{s} v_{s}\right)+\frac{\partial}{\partial x}\left[\phi_{s} p_{s}+\phi_{s} \rho_{s} v_{s}^{2}\right]-p_{g} \frac{\partial \phi_{s}}{\partial x} \\
\frac{\partial}{\partial t}\left(\phi_{g} \rho_{g} v_{g}\right)+\frac{\partial}{\partial x}\left[\phi_{g} p_{g}+\phi_{g} \rho_{g} v_{g}^{2}\right]+p_{g} \frac{\partial \phi_{s}}{\partial x}
\end{gathered}
$$

Also write down the overall equation

$$
\begin{aligned}
& \frac{\partial}{\partial t}\left[\phi_{s} p_{s} v_{s}+\phi_{g} p_{g} v_{g}\right]+\frac{\partial}{\partial x}\left[\left(\phi_{s} p_{s}+\phi_{g} p_{g}\right)\right. \\
& \left.+\left(\phi_{s} \rho_{s} v_{s}^{2}+\phi_{g} p_{g} v^{2}\right)\right]=0 .
\end{aligned}
$$

Energy

$$
\begin{aligned}
& \frac{\partial}{\partial t}\left[\phi_{s} \rho_{s}\left\{e_{s}+\frac{1}{2} v_{s}^{2}\right\}\right]+\frac{\partial}{\partial x}\left[\phi _ { s } \rho _ { s } v _ { s } \left\{e_{s}+\right.\right. \\
& \left.\left.+\frac{1}{2} v_{s}^{2}+\frac{p_{s}}{\rho_{s}}\right\}\right]=-\left(p_{s}-p_{s}\right) \mathcal{F}+p_{s} v_{s} \frac{\partial \phi_{s}}{\partial x}+\varepsilon
\end{aligned}
$$




$$
\begin{aligned}
& \quad \frac{\partial}{\partial t}\left[\phi_{g} \rho_{g}\left\{e_{g}+\frac{1}{2} v_{g}^{2}\right\}\right]+\frac{\partial}{\partial x}\left[\phi _ { g } \rho _ { g } v _ { g } \left\{e_{g}+\right.\right. \\
& \left.\left.+\frac{1}{2} v_{g}^{2}+\frac{p_{g}}{\rho_{g}}\right\}\right]=\left(p_{g}-\beta\right) \mathcal{F}-p_{g} v_{s} \frac{\partial \phi_{g}}{\partial x}-\varepsilon .
\end{aligned}
$$

Compaction

$$
\frac{\partial \phi_{s}}{\partial t}+v_{s} \frac{\partial \phi_{s}}{\partial x}=7+\frac{c}{p_{s}} \text {. }
$$

Interaction terms

$$
\begin{aligned}
& M=\delta\left(v_{g}-v_{s}\right)+\frac{1}{2} c\left(v_{g}+v_{g}\right) \\
& \mathcal{E}=\left(e_{s}+\frac{1}{2} v_{g}^{2}\right) c-H\left(T_{s}-T_{g}\right)+\left(\delta+\frac{1}{2} c\right) v_{s}\left(v_{g}-v_{s}\right. \\
& \mathcal{F}=\frac{\phi_{s} \phi_{g}}{\mu_{c}}\left(p_{s}-p_{g}-\beta_{s}\right)
\end{aligned}
$$

Oral energy eau.

$$
\begin{aligned}
& \frac{\partial}{\partial t}\left[\left(\phi_{s} p_{s} l_{s}+\phi_{g} p_{g} e_{g}\right)+\frac{1}{2}\left(\phi_{s} f_{s} v_{s}^{2}+\phi_{g} f_{g} v_{g}^{2}\right)\right] \\
& +\frac{\partial}{\partial x}\left[\left(\phi_{s} f_{s} v_{s} e_{s}+\phi_{g} p_{g} v_{g} l_{g}\right)+\frac{1}{2}\left(\phi_{s} f_{s} v_{s}^{3}+\phi_{g} p_{g} v_{g}^{3}\right)\right. \\
& \left.+\left(\phi_{s} v_{s} p_{s}+\phi_{g} v_{g} p_{g}\right)\right]=0
\end{aligned}
$$


(21)

Solid energy eau. - Vs $x$ solid momentum e egg. $\Rightarrow$

$$
\begin{gathered}
\frac{\partial}{\partial t}\left(\phi_{s} \rho_{s} e_{s}\right)+\frac{\partial}{\partial x}\left(\phi_{s} \rho_{s} v_{s} e_{s}\right)+\phi_{s} p_{s}\left(v_{s}\right), \\
=C e_{s}-H\left(T_{s}-T_{g}\right)-\left(p_{s}-p_{s}\right) \mathcal{F}
\end{gathered}
$$

Further simplification $\Rightarrow$

$$
\begin{aligned}
\phi_{s} f_{s}\left[\frac{\partial e_{s}}{\partial t}+v_{s} \frac{\partial e_{s}}{\partial x}\right] & =-\mu\left(T_{s}-T_{g}\right)-\left(p_{s}-\beta_{s}\right. \\
& -\phi_{s} p_{s} \frac{\partial v_{s}}{\partial x} .
\end{aligned}
$$

Note the absence of drag terms.

Gas entry eqn. - Vg $\times$ gas momentum e qu. $\Rightarrow$

$$
\begin{aligned}
\frac{\partial}{\partial t}\left(\phi_{g}\right. & \left.\rho_{g} e_{g}\right)+\frac{\partial}{\partial x}\left(\phi_{g} \rho_{g} v_{g} e_{g}\right)+\phi_{g} p_{g}\left(v_{g}\right) \\
& =H\left(T_{s}-T_{g}\right)+\left(p_{s}-\beta_{s}\right) f-C e_{s} \\
& +p_{g}\left(v_{g}-v_{s}\right) \frac{\partial \phi_{s}}{\partial x}+\delta\left(v_{g}-v_{s}\right)^{2}
\end{aligned}
$$

Note the asymmetry of these equations. Further simplification of the gas ign. $\Rightarrow$

$$
\begin{aligned}
& \phi_{g} f_{g}\left[\frac{\partial e_{g}}{\partial t}+v_{g} \frac{\partial e_{g}}{\partial x}\right]+\phi_{g} p_{g} \frac{\partial v_{g}}{\partial x}=c\left(e_{g}-t\right. \\
& -p_{g}\left(v_{g}-v_{s}\right) \frac{\partial \phi_{s}}{\partial x}+\delta\left(v_{g}-v_{s}\right)^{2}+H\left(T_{s}-T_{g}\right)+\left(\phi_{s}-\beta_{s}\right.
\end{aligned}
$$


4.

Wrue concider the limit $\delta \rightarrow \infty$. Eack of the two individual momentume equations, (1.3) and $(1.4)$, reduces to

$$
v_{s}-v_{g}=0 \Rightarrow v_{s}=v_{g}=v \quad \text { (i) }
$$

to leading acler. In reder to get a second independint equatione, one teress to the areerall momenture equ. (1.5), whick is independent of $S$.

The twe mass equs. sumanic the same, with the exceptrin of $v_{s}=v_{g}=25$. The envey equetions (1.6) and (2.7) do not simplify immediately beceuse each contains the $O$ (I) term $\delta\left(v_{g}-v_{s}\right)$. The ownall energy equ. (2.12) does, homenes, simplify.

The reduced salid eneygy equ. (3.1) a (3.2) simplifice pince $\delta$ daes nat appear there. The redreed gas encygy equ. (3.3) or $1(3.4)$ oimplifies, as mell, heeance $o$ is multiplied by $\left(v_{g}-v_{3}\right)^{2}$.

One my now wite doun the fallowing redued pet:

ass

$$
\begin{aligned}
& \frac{\partial}{\partial t}\left(\phi_{s} f_{t}\right)+\frac{\partial}{\partial x}\left(\phi_{s} f_{s} v\right)=C \\
& \frac{\partial}{\partial t}\left(\phi_{g} f_{g}\right)+\frac{\partial}{\partial x}\left(\phi_{g} f_{g} v\right)=-C
\end{aligned}
$$

nomentum

$$
\begin{gathered}
\frac{\partial}{\partial t}\left[\left(\phi_{s} \rho_{s}+\phi_{g} p_{g}\right) v\right]+\frac{\partial}{\partial x}\left[\left(\phi_{b} p_{g}+\phi_{g} p_{g}\right) v^{2}\right. \\
\left.+\left(\phi_{g} p_{s}+\phi_{g} p_{g}\right)\right]=0 .
\end{gathered}
$$


(13)

ency

$$
\begin{aligned}
\frac{\partial}{\partial t}\left(\phi_{s} \rho_{s} e_{s}\right) & +\frac{\partial}{\partial x}\left(\phi_{s} f_{s} e_{s} v\right)+\phi_{s} p_{s} v_{x} \\
& =C e_{s}-H\left(T_{s}-T_{g}\right)-\left(p_{s}-p_{s}\right) f \\
\frac{\partial}{\partial t}\left(\phi_{g} f_{g} e_{g}\right) & +\frac{\partial}{\partial x}\left(\phi_{g} f_{g} l_{g} v\right)+\phi_{g} p_{g} v_{x} \\
& =-C e_{s}+H\left(T_{s}-T_{g}\right)+\left(p_{s}-p_{s}\right) f
\end{aligned}
$$

Compaction

$$
\begin{aligned}
& \frac{\partial \phi_{s}}{\partial t}+v \frac{\partial \phi_{s}}{\partial x}=\mathcal{F}+\frac{c}{\rho_{s}} . \\
& \partial \frac{\partial}{\partial t}\left(\rho \phi_{s}\right)+\frac{\partial}{\partial x}\left(\rho \phi_{s} v\right)=\rho \mathcal{F}+\frac{\rho}{\rho_{s}} c
\end{aligned}
$$

Either of the two mass equs. may he replaced by an overall equation; simitar - Par energy.

The meal energy eau. can be written in a comenratinie forme by the individual energy equs. can nat, since the individual moment a ques. ane no longer avedilalle. 


\section{ITEM 7}




\section{Introduction}

In dimensional form, the basic equations are:

$$
\begin{array}{r}
\frac{\partial}{\partial t}\left(\phi_{s} \gamma_{s}\right)+\frac{\partial}{\partial x}\left(\phi_{s} \gamma_{s} u\right)=\mathcal{C}_{s} \\
\frac{\partial}{\partial t}\left(\phi_{g} \gamma_{g}\right)+\frac{\partial}{\partial x}\left(\phi_{g} \gamma_{g} u\right)=-\mathcal{C}_{s} \\
\frac{\partial}{\partial t}(\rho u)+\frac{\partial}{\partial x}\left(p+\rho u^{2}\right)=0 \\
\frac{\partial}{\partial t}\left(\phi_{s} \gamma_{s} e_{s}\right)+\frac{\partial}{\partial x}\left(\phi_{s} \gamma_{s} e_{s} u\right)=-\phi_{s} p_{s} \frac{\partial u}{\partial x}+\mathcal{C}_{s} e_{s}-H\left(T_{s}-T_{g}\right)-\left(p_{s}-\beta_{s}\right) \mathcal{F} \\
\frac{\partial}{\partial t}\left(\phi_{g} \gamma_{g} e_{g}\right)+\frac{\partial}{\partial x}\left(\phi_{g} \gamma_{g} e_{g} u\right)=-\phi_{g} p_{g} \frac{\partial u}{\partial x}-\mathcal{C}_{s} e_{s}+H\left(T_{s}-T_{g}\right)+\left(p_{s}-\beta_{s}\right) \mathcal{F} \\
\frac{\partial}{\partial t}\left(\rho \phi_{s}\right)+\frac{\partial}{\partial x}\left(\rho \phi_{s} u\right)=\rho \mathcal{F}+\frac{\rho}{\gamma_{s}} \mathcal{C}_{s}
\end{array}
$$

The mixture quantities appearing above are defined as

$$
\rho=\phi_{s} \gamma_{s}+\phi_{g} \gamma_{g}, \quad p=\phi_{s} p_{s}+\phi_{g} p_{g}
$$

The compaction function is

$$
\mathcal{F}=\frac{\phi_{s} \phi_{g}}{\mu_{c}}\left(p_{s}-p_{g}-\beta_{s}\right)
$$

\section{Solid EOS}

The equation of state for the solid is

$$
e_{s}=-T_{3}\left(1-\frac{1}{r}\right)+T_{4}\left\{r^{n-1}-(n-1)\left(1-\frac{1}{r}\right)-1\right\}+\frac{1}{G_{o v} r}\left\{p_{s}-p_{i n t}-\frac{A_{k t 0}}{n}\left(r^{n}-1\right)\right\}
$$

where $r$ is the density ratio

$$
r=\frac{\gamma_{s}}{\gamma_{s 0}}
$$

and the coefficients $T_{3}, T_{4}$ are given by

$$
T_{3}=\frac{c_{v s} T_{s 0} G_{o v}}{\gamma_{s 0}}, \quad T_{4}=\frac{A_{k t 0}}{n(n-1) \gamma_{s 0}}
$$

The eos yields the expression

$$
p_{s}-p_{i n t}=G_{o v} r\left[e_{s}+T_{3}\left(1-\frac{1}{r}\right)-T_{4}\left\{r^{n-1}-(n-1)\left(1-\frac{1}{r}\right)-1\right\}\right]+\frac{A_{k t 0}}{n}\left(r^{n}-1\right)
$$

for the pressure $p_{s}$ and

$$
c_{s}^{2}=\frac{1}{\partial e_{s} / \partial p_{s}}\left(\frac{p_{s}}{\gamma_{s}^{2}}-\frac{\partial e_{s}}{\partial \gamma_{s}}\right)
$$


for the solid sound speed, $c_{s}$. The temperature $T_{s}$ is given by

$$
T_{s}-T_{s 0}=\frac{1}{c_{v s}}\left[e_{s}+T_{3}\left(1-\frac{1}{r}\right)-T_{4}\left\{r^{n-1}-(n-1)\left(1-\frac{1}{r}\right)-1\right\}\right]
$$

In the MKS units, the coefficients in the eos have the values

$$
c_{v s}=0.15 e 4 \mathrm{Nm} / \text { Kg-degree K, } n=9.8, G_{o v}=2.1 \mathrm{e} 3 \mathrm{Kg} / \mathrm{m}^{3}, A_{k t 0}=1.35 \mathrm{e} 10 \mathrm{~N} / \mathrm{m}^{2}
$$

The initial values appearing above are taken to be

$$
T_{s 0}=300 \text { degrees } \mathrm{K}, \quad p_{\text {int }}=1.0 \mathrm{e} 5 \mathrm{~N} / \mathrm{m}^{2}, \gamma_{s 0}=1.9 \mathrm{es} \mathrm{K} / \mathrm{g}^{3} .
$$

The coefficients $T_{3}, T_{4}$ have units of energy per unit mass (ie, velocity squa, ${ }^{2}$ ).

\section{Gas EOS}

The easfor the gas is

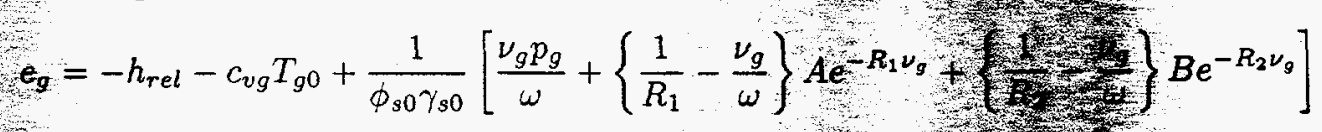

where

$$
\nu_{g}=\frac{\phi_{s 0} \gamma_{s 0}}{\gamma_{g}}
$$

Temperature $T_{g}$ is given by the expression

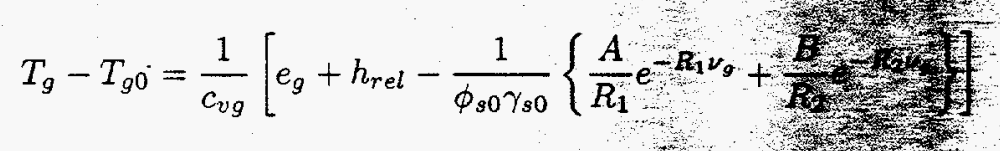

and pressure $p_{g}$ by

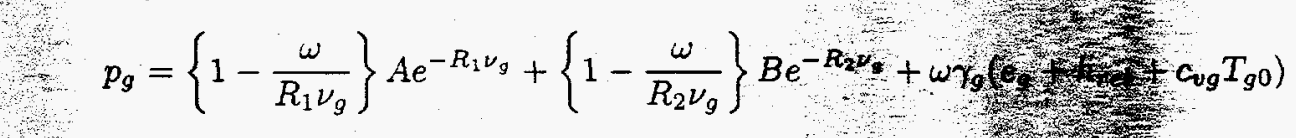

or equivalently, by

$$
p_{g}=A e^{-R_{1} \nu_{g}}+B e^{-R_{2} \nu_{g}}+\omega_{g} c_{g} T_{g}
$$

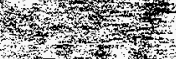

while the sound speed $c_{g}$ is computed from

$$
c_{g}^{2}=\frac{1}{\partial e_{g} / \partial p_{g}}\left(\frac{p_{g}}{\gamma_{g}^{2}}-\frac{\partial e_{g}}{\partial \gamma_{g}}\right)
$$

The dimensional coefficients appearing above have the following expressions th TIIKS units:

$$
\begin{aligned}
& c_{v g}=\left[2.4-0.28\left(10^{-3} \phi_{s 0} \gamma_{s 0}-1.3\right)\right] 10^{3} \text { \% } 1 \text { tring } / \text { Kg-degree K }
\end{aligned}
$$

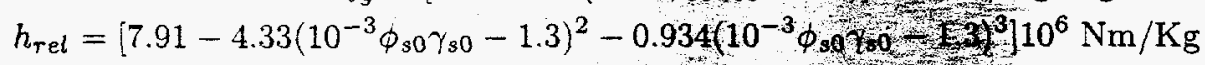

$$
\begin{aligned}
& A=\left[-8.005+21.39\left(10^{-3} \phi_{s 0} \gamma_{s 0}\right)-16.23\left(10^{-3} \phi_{s 0} \gamma_{s 0}\right)^{2}+4.595\left(10{ }^{3} \gamma_{00} \sigma_{60}\right){ }^{3}\right] 10^{11} \mathrm{~N} / \mathrm{m}^{2} \\
& B=\left[-0.014+0.0349\left(10^{-3} \phi_{s 0} \gamma_{s 0}\right)+0.0156\left(10^{-3} \phi_{s 0} \gamma_{s 0}\right)^{2}-0.026\left(10^{-3} \gamma_{0} \sigma_{00}\right)^{3}\right] 10^{11} \mathrm{~N} / \mathrm{m}^{2}
\end{aligned}
$$

The coefficients

$$
R_{1}=4.2, \quad R_{2}=1.0, \omega=0.25
$$

are dimensionless. Initial values are

$$
T_{g 0}=300 \text { degrees } \mathrm{K}, \quad p_{g 0}=1.0 e 5 \mathrm{~N} / \mathrm{m}^{2}
$$




\section{Compaction Law}

The compaction law is

$$
\beta_{s}= \begin{cases}-\tau\left(\phi_{s}-\phi_{s 0}\right) \frac{\ln \phi_{g}}{\phi_{g}} & \text { if } \phi_{s} \geq \phi_{s 0} \\ 0 & \text { otherwise }\end{cases}
$$

with the pressure coefficient

$$
\tau=1.2 e 7 \mathrm{~N} / \mathrm{m}^{2}
$$

and the compaction viscosity

$$
\mu_{c}=1.0 \mathrm{e} 2 \mathrm{Ns} / \mathrm{m}^{2}
$$

\section{Chemical Rate}

The chemical source term is specified as

$$
\mathcal{C}_{s}=-3 \gamma_{s} \phi_{s} A_{\text {coef }}\left(p_{\text {res }} / A_{\text {res }}\right)^{b_{n}} e_{t i}\left(1-f_{d v}\right)^{2 / 3} / a
$$

where

$$
\begin{gathered}
p_{r e s}=\operatorname{amax} 1\left(p_{g}, p_{i n t}\right), f_{d v}=\operatorname{amax} 1\left(\frac{\phi_{s}-\phi_{s 0}}{1-\phi_{s 0}}, 0\right) \\
a=(7.5 e-5) a \max 1\left(1, \phi_{s 0} / \phi_{s}\right)^{1 / 3} \mathrm{~m} .
\end{gathered}
$$

The coefficients $A_{\text {res }}, A_{\text {coef }}$ and $b_{n}$ are defined below:

$$
\begin{gathered}
A_{\text {res }}=1.0 e 8 \mathrm{~N} / \mathrm{m}^{2} \\
A_{\text {coef }}=0.1227 \mathrm{~m} / \mathrm{s}, \quad b_{n}=1 \text { for } p_{\text {res }} \leq 6.9 e 7 \mathrm{~N} / \mathrm{m}^{2} \\
A_{\text {coef }}=0.1139 \mathrm{~m} / \mathrm{s}, \quad b_{n}=0.8 \text { for } p_{\text {res }}>6.9 \mathrm{e} \mathrm{N} / \mathrm{m}^{2}
\end{gathered}
$$

Finally,

$$
e_{t i}= \begin{cases}0 & \text { for } p_{m i x}<p_{i g n} \\ 1 & \text { for } p_{m i x} \geq p_{i g n}\end{cases}
$$

where

$$
p_{\text {mix }}=p-p_{\text {int }}, \quad p_{\text {ign }}=1.0 e 7 \mathrm{~N} / \mathrm{m}^{2}
$$

\section{Scaling}

I prefer to use a scaled version of the equations for purposes of computation. Let the basic scales be $x_{*}$ for length, $t_{*}$ for time, $\gamma_{*}$ for density and $c_{*}$ for specific heat. Scales for the remaining variables are taken to be $u_{*}=x_{*} / t_{*}$ for speed, $p_{*}=\gamma_{*} u_{*}^{2}$ for pressure, $e_{*}=u_{*}^{2}$ for internal energy and $T_{*}=u_{*}^{2} / c_{*}$ for temperature. The chemical source term has the scale $\mathcal{C}_{s *}=\gamma_{*} / t_{*}$, and the compaction term scales as $\mathcal{F}_{*}=1 / t_{*}$.

The scales for the compaction viscosity and the convection heat transfer coefficient are $\mu_{c *}=\gamma_{*} u_{*}^{2} t_{*}$ and $H_{*}=\gamma_{*} c_{*} / t_{*}$

With these choices, the governing equations $(1)-(8)$ remain as before, with the symbols now denoting scaled variables. The solid EOS is again represented by (9), with $G_{o v}$ scaled by $\gamma_{*}, T_{3}$ and $T_{4}$ by $u_{*}^{2}$ and $A_{k t 0}$ by $p_{*}$. The sound speed formula (13) and the temperature equation (14) survive unscathed. 
The gas EOS (17), the temperature equation (19) and the pressure equations (20), (21) also remain unchanged, provided $A$ and $B$ are scaled by $p_{*}$. The sound-speed equation (22) holds as well.

In $(29), \tau$ is scaled by $p_{*}$.

In the chemical rate term, given by (32), $A_{\text {coef }}$ is scaled by $u_{*}$ and $a$ by $x_{*}$. The pressure $p_{\text {res }}$ and the coefficient $A_{\text {res }}$ are, of course, scaled by $p_{*}$. 
Nov 29,1993

Sound-speed computation (revised)

I. SOLID

The solid eos may be written as follows:

Let $r=r_{5} / r_{s o}$.

(1)

Then,

$$
\begin{aligned}
e_{s}= & -T_{3}\left(1-\frac{1}{2}\right)+T_{4}\left\{R^{n-1}-(n-1)\left(1-\frac{1}{2}\right)-1\right\} \\
& +\frac{1}{G_{\text {or }}} \frac{1}{2}\left[p_{s}-p_{\text {int }}-A_{k t 0} \frac{1}{n}\left(\kappa^{n}-1\right)\right]
\end{aligned}
$$

Now,

$$
r_{s} c_{s}^{2}=\frac{1}{\partial e_{s} / \partial p_{s}}\left[\frac{p_{s}}{r_{s}}-r_{s} \frac{\partial e_{s}}{\partial r_{s}}\right]
$$

Nous,

$$
\begin{aligned}
& \frac{\partial e_{s}}{\partial p_{s}}=\frac{1}{G_{o v}} \frac{1}{2} \\
& \frac{\partial e_{s}}{\partial r_{s}}=\frac{1}{r_{s o}} \frac{\partial e_{s}}{\partial r} \\
& =\frac{1}{r_{s 0}}\left[-\frac{T_{3}}{r^{2}}+T_{4}\left\{(n-1) r^{n-2}-\frac{n-1}{r^{2}}\right\}\right. \\
& -\frac{1}{\operatorname{Gar}} \frac{1}{\kappa^{2}}\left\{p_{s}-p_{\text {int }}-\frac{A_{k t}}{n}\left(\kappa^{n}-1\right)\right\} \\
& \left.+\frac{1}{G_{v v} r}\left(-A_{k} t_{0}\right) \kappa^{n-1}\right] \\
& =\frac{1}{r_{s_{0}} \kappa^{2}}\left[-T_{3}+(n-1) T_{4}\left(\kappa^{n}-1\right)-\right. \\
& \left.-\frac{1}{G_{\text {on }}}\left\{p_{s}-p_{\text {int }}-\frac{A_{k t_{0}}}{n}\left(r^{n}-1\right)\right\}-\frac{A_{k t}}{G_{0 V}} r^{n}\right](5)
\end{aligned}
$$


(9)

$$
\begin{aligned}
& \left.\left.\frac{m}{z y}-\frac{m}{1}\right\}_{\sigma_{s} z y} \partial \theta-\left\{1+\frac{m}{\sigma_{1} y}-\frac{m}{1}\right\}_{\sigma_{x} y-} \partial t-\frac{m}{6 f}\right] \frac{z^{6} x}{1}=
\end{aligned}
$$

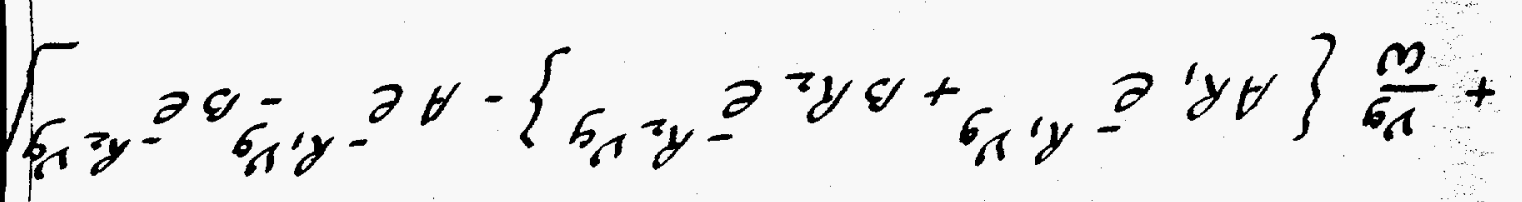

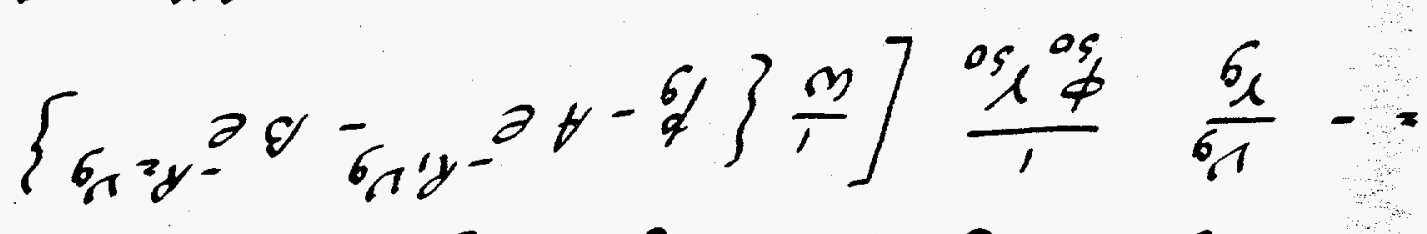

$$
\frac{\sigma_{r e}}{\sigma_{\partial}} \frac{\sigma_{x}}{\sigma_{1}}=\frac{\sigma_{x e}}{\sigma_{1}} \frac{\sigma_{1}}{\sigma_{\partial e}}=\frac{\sigma_{x e}}{\sigma_{\partial e}}
$$

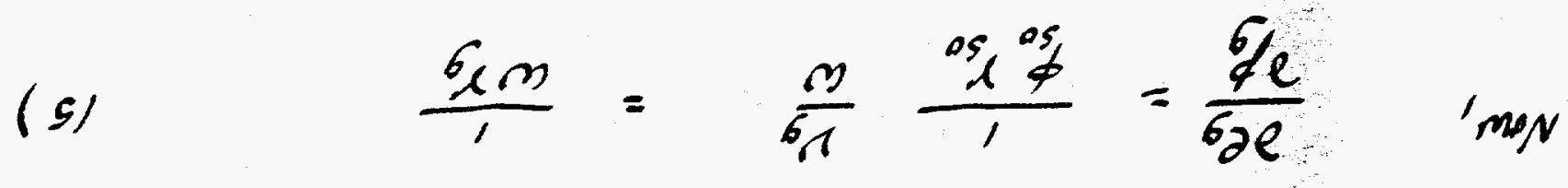

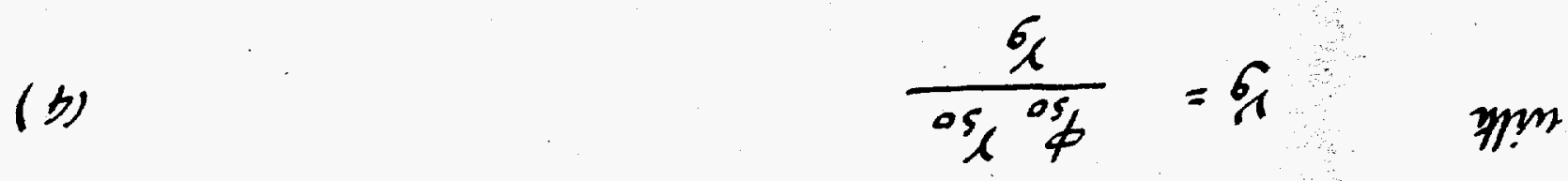

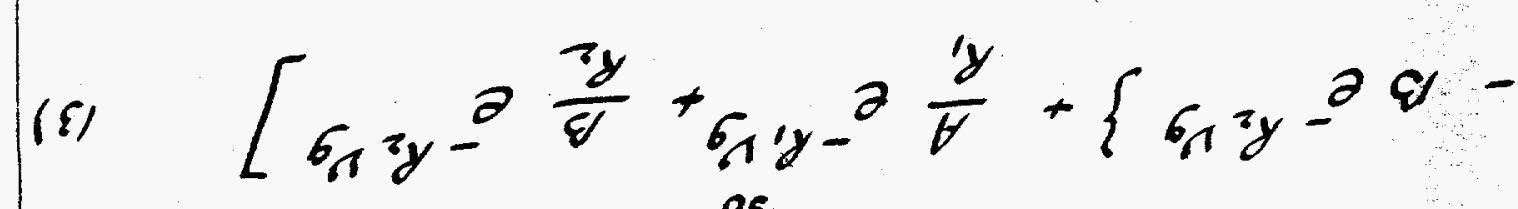

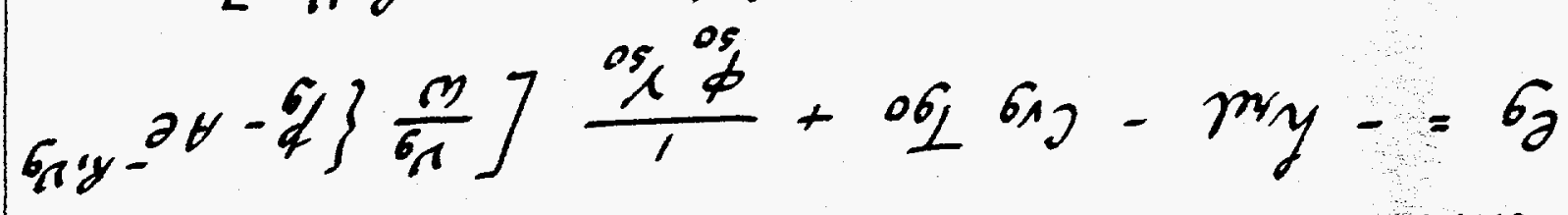

SHY II

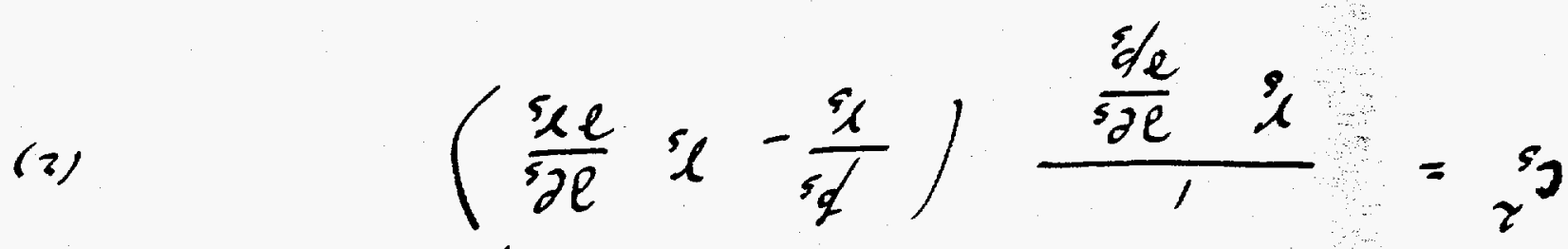

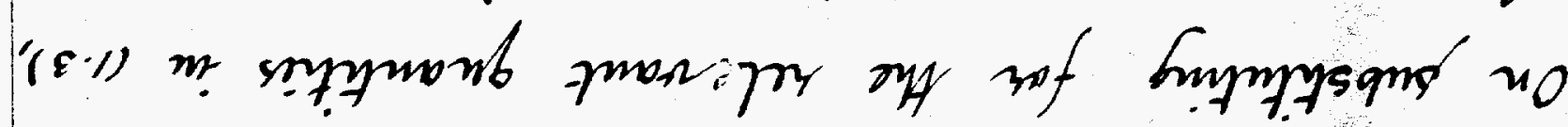

(1) $\left[\left\{1-\left(\frac{n}{i}-1\right)(1-u)-1-u^{2}\right\} b_{1}-\right.$

$$
\left.-\left(\frac{y}{1}-1\right) \varepsilon_{L}+s_{2}\right] \operatorname{sog} y+\left(1-w^{y}\right) \frac{u}{y+t}+p^{m} q=q
$$

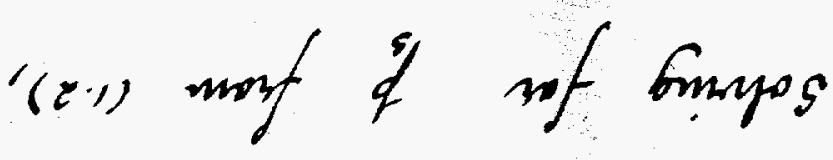

$\varepsilon b / 6 c / 11$ 
$11 / 29 / 93$

3.

The gas sound speed may now be compete from

$$
c_{g}^{2}=\frac{1}{r_{g} \frac{\partial e_{g}}{\partial p_{g}}}\left\{\frac{p_{g}}{r_{g}}-r_{g} \frac{\partial g}{\partial r_{g}}\right]
$$

Finally, the mixture speed is gif w by

$$
c_{m}^{2}=\frac{\phi_{g} r_{g} c_{g}^{2}+\phi_{s} r_{s} c_{s}^{2}}{\phi_{g} r_{g}+\phi_{s} r_{s}}
$$


Alternate Scaling (On t rather than $x$ )

velocity scale $u_{*}$, length scale $l_{*}=1$

time scale $t_{*}=l_{*} / u_{*}=1 / u_{*}$

pressure $t_{*}=U_{*}^{2}$, also $e_{*}=T_{*}=U_{*}^{2}$

Mass equations

$$
\begin{aligned}
& \frac{\partial}{\partial \bar{t}}\left(\phi_{s} \gamma_{s}\right)+\frac{\partial}{\partial x}\left(\phi_{s} \gamma_{s} \bar{u}\right)=\bar{C}_{s} \\
& \frac{\partial}{\partial \bar{t}}\left(\phi_{g} \gamma_{g}\right)+\frac{\partial}{\partial x}\left(\phi_{g} \gamma_{g} \bar{u}\right)=-\bar{C}_{s}
\end{aligned}
$$

Momentum

$$
\frac{\partial}{\partial \bar{t}}(\rho \bar{u})+\frac{\partial}{\partial x}\left(\rho \bar{u}^{2}+\bar{p}\right)=0
$$

Compaction

$$
\frac{\partial}{\partial \bar{E}}\left(\rho \phi_{s}\right)+\frac{\partial}{\partial x}\left(\rho \phi_{s} \bar{u}\right)=\rho \bar{F}+\frac{\rho}{\gamma_{s}} \overline{C_{s}}
$$

Energy

$$
\begin{aligned}
& \frac{\partial}{\partial \bar{t}}\left(\phi_{s} \gamma_{s} \bar{e}_{s}\right)+\frac{\partial}{\partial x}\left(\phi_{s} \gamma_{s} \bar{e}_{s} \bar{u}\right)+\phi_{s} \bar{p}_{s} \frac{\partial \bar{u}}{\partial x} \\
& =\bar{C}_{s} \bar{e}_{s}-\bar{H}\left(\bar{T}_{s}-\bar{T}_{g}\right)-\left(\bar{F}_{s}-\bar{\beta}_{s}\right) \overline{\mathcal{F}} \\
& \frac{\partial}{\partial t}\left(\phi_{g} \gamma_{g} \bar{l}_{g}\right)+\frac{\partial}{\partial x}\left(\phi_{g} \gamma_{g} \bar{e}_{g} \bar{u}\right)+\phi_{g} \bar{\phi}_{g} \frac{\partial \bar{u}}{\partial x} \\
& =-\bar{C}_{s} \bar{e}_{s}+\bar{H}\left(\bar{T}_{s}-\bar{T}_{g}\right)-\left(\bar{p}_{s}-\bar{\beta}_{s}\right) \\
& \bar{H}=H / U_{*}, \quad \bar{F}=\frac{\phi_{s} \phi_{g} U_{*}}{\mu_{c}}\left(\bar{p}_{s}-\bar{\beta}_{g}-\bar{\beta}_{s}\right) \\
& \bar{c}_{s}=c_{s} / U_{*}, \quad \bar{t}=t U_{*}
\end{aligned}
$$


The equal-velocity model (Kapila notation)

$$
\begin{aligned}
& \frac{\partial}{\partial t}\left(\phi_{s} \gamma_{s}\right)+\frac{\partial}{\partial x}\left(\phi_{s} \gamma_{s} v\right)=C_{s} \\
& \frac{\partial}{\partial t}\left(\phi_{g} \gamma_{g}\right)+\frac{\partial}{\partial x}\left(\phi_{g} \gamma_{g} v\right)=-C_{s} \\
& \frac{\partial}{\partial t}(\rho v)+\frac{\partial}{\partial x}\left(\rho v^{2}+p\right)=0 \\
& \frac{\partial}{\partial t}\left(\phi_{s} \gamma_{s} e_{s}\right)+\frac{\partial}{\partial x}\left(\phi_{s} r_{s} e_{s} v\right)+\phi_{s} p_{s} \frac{\partial v}{\partial x} \\
& =C_{s} e_{s}-H\left(T_{s}-T_{g}\right)-\left(p_{s}-\beta_{s}\right) \mathcal{F} \\
& \frac{\partial}{\partial t}\left(\phi_{g} \gamma_{g} e_{g}\right)+\frac{\partial}{\partial x}\left(\phi_{g} r_{g} e_{g} v+\phi_{g} p_{g} \frac{\partial v}{\partial x}\right. \\
& =-C_{s} e_{s}+H\left(T_{s}-T_{g}\right)+\left(p_{s}-\beta_{s}\right) \mathcal{F} \\
& \frac{\partial}{\partial t}\left(\rho \phi_{s}\right)+\frac{\partial}{\partial x}\left(\rho \phi_{s} v\right)=\rho \mathcal{F}+\frac{\rho}{\gamma_{s}} C_{s} \\
& \rho=\phi_{s} \gamma_{s}+\phi_{g} \gamma_{g}, \quad p=\phi_{s} p_{s}+\phi_{g} p_{g} \\
& \mathcal{F}=\frac{\phi_{s} \phi_{g}}{\mu_{c}}\left(p_{s}-p_{g}-\beta_{s}\right)
\end{aligned}
$$


Scaled Equations

Take time scale $t_{*}=1$

$$
\begin{aligned}
& \text { velocity scale }=u_{*} \\
& \text { length scale }=u_{*} t_{*}=U_{*} \\
& \text { pressure peale } \phi_{*}=u_{*}^{2}
\end{aligned}
$$

energy scale $e_{*}=U_{*}^{2}=$ temp. scale $T_{*}$

Let $u=u_{*} \bar{u}, p=u_{*}^{2} \bar{p}$, ck

Mass equs. are unchanged.

$$
\begin{aligned}
& \frac{\partial}{\partial t}\left(\phi_{s} \gamma_{s}\right)+\frac{\partial}{\partial \bar{x}}\left(\phi_{s} \gamma_{s} \bar{u}\right)=C_{s} \\
& \frac{\partial}{\partial t}\left(\phi_{g} \gamma_{g}\right)+\frac{\partial}{\partial \bar{x}}\left(\phi_{g} \gamma_{g} \bar{u}\right)=-C_{s} .
\end{aligned}
$$

Momentum e eau. is unchanged.

$$
\frac{\partial}{\partial t}(\rho \bar{u})+\frac{\partial}{\partial \bar{x}}\left(\rho \bar{u}^{2}+\bar{p}\right)=0 .
$$

Energy equo:

$$
\begin{aligned}
\frac{\partial}{\partial t}\left(\phi_{s} \gamma_{s} \bar{e}_{s}\right) & +\frac{\partial}{\partial \bar{x}}\left[\phi_{s} \gamma_{s} \bar{e}_{s} \bar{u}\right]+\phi_{s} \overline{p_{s}} \frac{\partial \bar{u}}{\partial \bar{x}} \\
& =C_{s} \overline{e_{s}}-H\left(\overline{T_{s}}-\overline{T_{g}}\right)-\left(\overline{p_{s}}-\bar{\beta}\right) \bar{s}
\end{aligned}
$$




$$
\begin{array}{r}
\frac{\partial}{\partial t}\left(\phi_{g} r_{g} \bar{e}_{g}\right)+\frac{\partial}{\partial \bar{x}}\left[\phi_{g} \gamma_{g} \bar{e}_{g} \bar{u}\right]+\phi_{g} \overline{p_{g}} \frac{\partial \bar{u}}{\partial \bar{x}} \\
=-c_{s} \bar{e}_{s}+H\left(\overline{\bar{t}_{s}}-\overline{\bar{T}_{g}}\right)+\left(\overline{\bar{\beta}_{s}}-\bar{\beta}_{s}\right) \mathcal{F}
\end{array}
$$

compaction

$$
\begin{aligned}
& \frac{\partial}{\partial t}\left(\rho \phi_{s}\right)+\frac{\partial}{\partial \bar{x}}\left(\rho \phi_{s} \bar{u}\right)=\rho \bar{y}+\frac{\rho}{\gamma_{s}} C_{s} \\
& \rho=\phi_{s} \gamma_{s}+\phi_{g} \gamma_{g} \\
& \bar{F}=\phi_{s} \bar{p}_{s}+\phi_{g} \bar{p}_{g} \\
& \bar{F}=\frac{\phi_{s} \phi_{g} u_{*}^{2}}{\mu_{c}}\left[\overline{p_{s}}-\bar{\beta}-\bar{\beta}\right]
\end{aligned}
$$


Machine Notation

$$
\begin{aligned}
& \text { rho }(1, n)=r_{s} \phi_{s}, \text { rho }(2, n)=r_{g} \phi_{g} \\
& \text { rhou }(n)=\left(r_{s} \phi_{s}+r_{g} \phi_{g}\right) u \\
& \text { rhophi }(1, n)=\left(r_{s} \phi_{s}+r_{g} \phi_{g}\right) \phi_{s} \\
& \text { rhoe }(1, n)=\left(r_{s} \phi_{s}+r_{g} \phi_{g}\right) e_{s} \\
& \text { rhoe }(2, n)=\left(r_{s} \phi_{s}+r_{g} \phi_{g}\right) e_{g} \\
& \text { p }(1, n)=p_{s}, \quad p(2, n)=g_{g} \\
& \text { temp }(1, n)=T_{s}, \quad \operatorname{temp}(2, n)=T_{g} .
\end{aligned}
$$

gamaso, gamago, phiso, phigo

$$
\begin{aligned}
& r_{s o} \quad r_{90} \quad \phi_{s o} \quad \phi_{90} \\
& \begin{array}{llll}
\text { tso } & t_{90} & \text { rhoso } & \text { shogo } \\
T_{\text {so }} & T_{90} & \gamma_{\text {so }} \phi_{s 0} & Y_{90} \phi_{90}
\end{array} \\
& \text { den }=\gamma_{s} \phi_{s}+\gamma_{g} \phi_{g}, u=\text { rhoufden, } \phi_{s}=\text { shophi, }
\end{aligned}
$$

The 6 basic variables are tho $(1, n)$, who $(2, n)$, thou(n), thee $(1, n)$, thoe $(2, n$ thophi $(1, x)$ 